\title{
NUMERICAL ANALYSIS OF A FINITE ELEMENT SCHEME FOR THE APPROXIMATION OF HARMONIC MAPS INTO SURFACES
}

\author{
SÖREN BARTELS
}

\begin{abstract}
This article studies the numerical approximation of harmonic maps into surfaces, i.e., critical points for the Dirichlet energy among weakly differentiable vector fields that are constrained to attain their pointwise values in a given manifold. An iterative algorithm that is based on a linearization of the constraint about the current iterate at the nodes of a triangulation is devised, and its global convergence to a discrete harmonic map is proved under general conditions. Weak accumulation of discrete harmonic maps at harmonic maps as discretization parameters tend to zero is established in two dimensions under certain assumptions on the underlying sequence of triangulations. Numerical simulations illustrate the performance of the algorithm for curved domains.
\end{abstract}

\section{INTRODUCTION}

Geometric partial differential equations and their analysis as well as numerical simulation have recently attracted considerable attention among pure and applied mathematicians. Motivated by interesting applications such as general relativity, micromagnetics, liquid crystal theory, biophysics, and medical image processing, significant progress has been made in the mathematical understanding of evolutionary and stationary partial differential equations on, into, and between surfaces within the last two decades. While the properties and approximation of solutions of partial differential equations with values into surfaces with symmetries such as the unit sphere are now relatively well understood, only a few results are available in the case of a general target manifold.

In the so-called equal-constant setting in liquid crystal theory or in models for ferromagnetic bodies of small diameter, the Oseen-Frank energy [35, 22, 42, 16, 28, [20, 3] or the Landau-Lifschitz energy [29, 18], respectively, reduces to the Dirichlet energy

$$
E(v)=\frac{1}{2} \int_{M}|\nabla v|^{2} \mathrm{~d} x,
$$

and one is led to minimizing $E$ among vector fields $v: M \rightarrow S^{2}$ or $v: M \rightarrow \mathbb{R} P^{2}$, the two-dimensional unit sphere or the real projective plane. In this paper, we will consider as a target manifold a compact, $k$-dimensional $C^{2}$ submanifold $N \subset \mathbb{R}^{n}$ without boundary. This excludes $\mathbb{R} P^{2}$ but still allows for a variety of interesting

Received by the editor December 1, 2008 and, in revised form, May 8, 2009.

2000 Mathematics Subject Classification. Primary 65N12, 65N22, 58E20.

(C)2009 American Mathematical Society 
applications. A weakly differentiable vector field $u: M \rightarrow N$ will be called a harmonic map into $N$ if it is stationary for $E$ with respect to compactly supported, tangential perturbations. This is true if $u$ is a weak solution of the nonlinear partial differential equation

$$
-\Delta u=\sum_{\gamma=1}^{d} A_{N}(u)\left[\partial_{\gamma} u ; \partial_{\gamma} u\right]
$$

with the second fundamental form $A_{N}$ on $N$ and $d:=\operatorname{dim}(M)$; see, e.g., [40, 23].

The existence of global minimizers for (11) and hence of harmonic maps into $N$ follows with the direct method in the calculus of variations for nonempty sets of admissible vector fields. The simplest choice of a nonflat target manifold already reveals a variety of intriguing phenomena captured by the mathematical model (1): If $M$ is two-dimensional, then harmonic maps are known to be smooth; see [25, 33. [26, 37. In higher dimensions the picture changes drastically. For $d=3$ harmonic maps into the sphere are partially regular if they are energy minimizing or, more generally, stationary with respect to spatial variations $21,10,24$. Those results are sharp in the sense that the function $x \mapsto x /|x|$ is an energy minimizing harmonic map into the sphere if $d \geq 3$ [30, 11, 27, 39], and there exist harmonic maps into $S^{n-1}$ which are everywhere discontinuous if $d, n \geq 3$ [36].

The properties of harmonic maps indicate that approximation schemes have to be developed carefully in order to deal with the limited regularity. The three major difficulties in the numerical approximation are that numerical schemes have to (i) cope with the critical nonlinearity in the right-hand side of (2), (ii) satisfy the constraint $u(x) \in N$ appropriately, and (iii) lead to approximations of low energy.

The first issue (i) can be effectively solved by noting that $A_{N}$ assumes values in the normal bundle of $N$ and restricting to test functions that are tangential along the unknown $u$. This results in the equivalent weak formulation of finding a weakly differentiable $u: M \rightarrow N$ such that

$$
(\nabla u ; \nabla v)=0
$$

for all smooth vector fields $v: M \rightarrow \mathbb{R}^{n}$ satisfying $v(x) \in T_{u(x)} N$ for almost every $x \in M$ and where $(\cdot ; \cdot)$ denotes the inner product in $L^{2}\left(M ; \mathbb{R}^{n}\right)$. The practical realization (ii) of the constraint $u(x) \in N$ is by no means a straightforward task. Even for the simplest case $N=S^{n-1}$ one easily verifies that a continuous, piecewise polynomial function $w_{h}$ satisfies $w_{h}(x) \in S^{n-1}$ for almost every $x \in M$, i.e., $\left|w_{h}\right|=1$ almost everywhere in $M$, if and only if $w_{h}$ is constant. Therefore, approximation schemes relax the constraint, and finite element approximations are only required to assume their nodal values in $N$. In this way, the constraint may be satisfied almost nowhere, but for a bounded sequence of such finite element functions every weak accumulation point satisfies the constraint almost everywhere. Ginzburg-Landau approximations provide another way of imposing the constraint in a relaxed, practical way. This, however, requires the introduction of a small penalization parameter and the solutions of the resulting regularized problem usually do not show the desired sharp topological effects. Finally, the problem (iii) of computing harmonic maps of low energy can be solved by discretizing gradient flows of harmonic maps and choosing discretization parameters such that discrete energy laws are satisfied.

Given a regular triangulation $\mathcal{T}_{h}$ of $M$ with nodes contained in $\mathcal{N}_{h}$ and a subordinated lowest order finite element space $\mathcal{S}^{1}\left(\mathcal{T}_{h}\right)$, the aforementioned ideas motivate 
the following definition: The vector field $u_{h} \in \mathcal{S}^{1}\left(\mathcal{T}_{h}\right)^{n}$ is called a discrete harmonic map into $N$ subject to the boundary data $u_{\mathrm{D}}$ if $\left.u_{h}\right|_{\Gamma_{\mathrm{D}}}=u_{\mathrm{D}, h}, u_{h}(z) \in N$ for all $z \in \mathcal{N}_{h}$, and

$$
\left(\nabla u_{h} ; \nabla v_{h}\right)=0
$$

is satisfied for all vector fields $v_{h} \in \mathcal{S}^{1}\left(\mathcal{T}_{h}\right)^{n}$ such that $\left.v_{h}\right|_{\Gamma_{\mathrm{D}}}=0$ and $v_{h}(z) \in$ $T_{u_{h}(z)} N$ for all $z \in \mathcal{N}_{h}$. Here, we included Dirichlet's conditions on the possibly empty subset $\Gamma_{\mathrm{D}} \subseteq \partial M$. It is our aim to iteratively compute discrete harmonic maps and to show that sequences of discrete harmonic maps accumulate at harmonic maps as the maximal mesh-size $h$ tends to zero.

The approximation of harmonic maps into spheres started with the work 31 that studied point relaxation methods. An energy decreasing iterative algorithm that linearizes the constraint in each step has been introduced and shown to converge in a continuous setting in [1. Convergence of a finite element discretization of that algorithm on weakly acute triangulations has been established in 6. The authors of 41 discuss parametric approaches for the approximation of $p$-harmonic maps into spheres that lead to unconstrained discrete problems and successfully employ them to denoise color images. An interesting saddle-point formulation for the computation of discrete harmonic maps into spheres that leads to a separately convex optimization problem has been proposed in [14]. Various methods for the discretization of the harmonic map heat flow into spheres have recently been developed and analyzed in [4, 9, 2,

Apart from the convergence result in [34 for discrete harmonic maps on planar, regular lattices to harmonic maps into compact $C^{4}$ submanifolds $N \subset \mathbb{R}^{n}$ without boundary, the author is unaware of algorithms or approximation results for discrete harmonic maps into general target manifolds.

Motivated by the definition of a discrete harmonic map into a given surface $N$ and generalizing work of [1, 7, 4, 9] for $N=S^{n-1}$, we employ the following iteration to compute discrete harmonic maps of low energy. We denote by $\mathcal{S}_{\mathrm{D}}^{1}\left(\mathcal{T}_{h}\right)$ the subspace of $\mathcal{S}^{1}\left(\mathcal{T}_{h}\right)$ consisting of functions that vanish on $\Gamma_{\mathrm{D}}$ if this set is nonempty and has zero integral mean otherwise; $\pi_{N}$ denotes the nearest-neighbour projection onto $N$ which is well defined in a small, tubular neighbourhood of $N$ provided that $N$ is $C^{2}$.

Algorithm A. Input: triangulation $\mathcal{T}_{h}$, damping parameter $\kappa>0$, stopping criterion $\varepsilon>0$.

(1) Choose $u_{h}^{0} \in \mathcal{S}^{1}\left(\mathcal{T}_{h}\right)^{n}$ such that $\left.u_{h}^{0}\right|_{\Gamma_{\mathrm{D}}}=u_{\mathrm{D}, h}$ and $u_{h}^{0}(z) \in N$ for all $z \in$ $\mathcal{N}_{h} \backslash \Gamma_{\mathrm{D}}$. Set $i:=0$.

(2) Compute $w_{h}^{i} \in \mathcal{S}_{\mathrm{D}}^{1}\left(\mathcal{T}_{h}\right)^{n}$ such that $w_{h}^{i}(z) \in T_{u_{h}^{i}(z)} N$ for all $z \in \mathcal{N}_{h}$ and

$$
\left(\nabla w_{h}^{i} ; \nabla v_{h}\right)=-\left(\nabla u_{h}^{i} ; \nabla v_{h}\right)
$$

for all $v_{h} \in \mathcal{S}_{\mathrm{D}}^{1}\left(\mathcal{T}_{h}\right)^{n}$ such that $v_{h}(z) \in T_{u_{h}^{i}(z)} N$ for all $z \in \mathcal{N}_{h}$.

(3) Stop if $\left\|\nabla w_{h}^{i}\right\| \leq \varepsilon$.

(4) Define $u_{h}^{i+1} \in \mathcal{S}^{1}\left(\mathcal{T}_{h}\right)^{n}$ by setting for all $z \in \mathcal{N}_{h}$,

$$
u_{h}^{i+1}(z):=\pi_{N}\left(u_{h}^{i}(z)+\kappa w_{h}^{i}(z)\right) .
$$

(5) Set $i:=i+1$ and go to (2).

Output: $u_{h}^{*}:=u_{h}^{i}$. 
Algorithm A can be derived by discretizing the $H^{1}$ gradient flow of harmonic maps into $N$, and then $\kappa$ is a time-step size while $w_{h}^{i}$ serves as an approximation of the time-derivative. Another motivation of the iteration results from regarding $w_{h}^{i}$ as a correction of the given approximation $u_{h}^{i}$ and a linearization of the condition $\widetilde{u}_{h}^{i+1}=u_{h}^{i}(z)+\kappa w_{h}^{i}(z) \in N$ about $u_{h}^{i}(z)$. In this case $\kappa$ is a damping parameter which is needed to guarantee that the nodal values of the update $u_{h}^{i}+\kappa w_{h}^{i}$ belong to $U_{\delta_{N}}(N)$ so that Step (4) in the loop is well defined. The following theorem states that the iteration is well defined and convergent if $\kappa=\mathcal{O}\left(h_{\text {min }}\right)$ with $h_{\min }$ denoting the minimal diameter of elements in $\mathcal{T}_{h}$.

Theorem I. Suppose that $N$ is $C^{3}$. There exist $h$-independent constants $C^{\prime}, C^{\prime \prime}>0$ such that if $\kappa \leq C^{\prime} h_{\min }$ and $\varepsilon>0$, then Algorithm $A$ is feasible and terminates within a finite number of iterations. The output $u_{h}^{*}$ satisfies $u_{h}^{*}(z) \in N$ for all $z \in \mathcal{N}_{h},\left.u_{h}^{*}\right|_{\Gamma_{\mathrm{D}}}=u_{\mathrm{D}, h},\left\|\nabla u_{h}^{*}\right\| \leq C^{\prime \prime}\left\|\nabla u_{h}^{0}\right\|$, and

$$
\left|\left(\nabla u_{h}^{*} ; \nabla v_{h}\right)\right| \leq \varepsilon\left\|\nabla v_{h}\right\|
$$

for all $v_{h} \in \mathcal{S}_{\mathrm{D}}^{1}\left(\mathcal{T}_{h}\right)^{n}$ such that $v_{h}(z) \in T_{u_{h}^{*}(z)} N$ for all $z \in \mathcal{N}_{h}$.

The assumptions of Theorem I can be significantly weakened if $N=\partial \mathcal{C}$ for a bounded, open, convex set $\mathcal{C} \subset \mathbb{R}^{n}$ and if $\mathcal{T}_{h}$ is weakly acute. In this case, using that $\pi_{N}: \mathbb{R}^{n} \backslash \mathcal{C} \rightarrow N$ is nonexpanding, $\kappa$ can be chosen of order one and we have $C^{\prime \prime}=1$. It is worth remarking that Algorithm A is globally convergent. While this guarantees that any choice of $u_{h}^{0}$ will lead to a discrete harmonic map into $N$, it also explains that the iteration can be slowly convergent.

The proof of Theorem I exploits the fact that Algorithm A can be understood as a discretization of the $H^{1}$ gradient flow of harmonic maps and that $\pi_{N}$ is $C^{2}$ with $\left.D \pi_{N}(p)\right|_{T_{p} N}=\left.\mathrm{id}\right|_{T_{p} N}$ for all $p \in N$. Proving convergence of a sequence of outputs of Algorithm A to a harmonic map into $N$ as $h, \varepsilon \rightarrow 0$ is more involved and we provide an affirmative answer if $M$ is two-dimensional and the sequence of triangulations satisfies a restrictive angle condition: $\left(\mathcal{T}_{h}\right)_{h>0}$ is said to be logarithmically right-angled if for every $\varepsilon>0$ there exists $h_{0}>0$ such that for all $0<h \leq h_{0}$ and every triangle $K \in \mathcal{T}_{h}$ with inner angles $\alpha_{K, j} \in(0, \pi), j=1,2,3$, we have $\min _{j=1,2,3} \log h_{\min }^{-1}\left|\cos \alpha_{K, j}\right| \leq \varepsilon$. Sufficient for this is that for every $h>0$ each $K \in \mathcal{T}_{h}$ has a right angle.

Theorem II. Suppose that $d=2$ and $N$ is $C^{4}$. Let $\left(\mathcal{T}_{h}\right)_{h>0}$ be a sequence of regular, logarithmically right-angled triangulations and for each $h>0$, let $u_{h} \in$ $\mathcal{S}^{1}\left(\mathcal{T}_{h}\right)^{n}$ satisfy $\left.u_{h}\right|_{\Gamma_{\mathrm{D}}}=u_{\mathrm{D}, h}$ and $u_{h}(z) \in N$ for all $z \in \mathcal{N}_{h}$. Assume that for each $h>0$ there exists $\varepsilon_{h}>0$ such that we have

$$
\left|\left(\nabla u_{h} ; \nabla v_{h}\right)\right| \leq \varepsilon_{h}\left\|\nabla v_{h}\right\|
$$

for all $v_{h} \in \mathcal{S}_{\mathrm{D}}^{1}\left(\mathcal{T}_{h}\right)^{n}$ satisfying $v_{h}(z) \in T_{u_{h}(z)} N$ for all $z \in \mathcal{N}_{h}$. If $\left\|\nabla u_{h}\right\| \leq C$, $u_{\mathrm{D}, h} \rightarrow u_{\mathrm{D}}$ in $L^{2}\left(\Gamma_{\mathrm{D}} ; \mathbb{R}^{n}\right)$, and $\varepsilon_{h} \rightarrow 0$ as $h \rightarrow 0$, then every weak accumulation point $u \in W^{1,2}\left(M ; \mathbb{R}^{n}\right)$ of $\left(u_{h}\right)_{h>0} \subset W^{1,2}\left(M ; \mathbb{R}^{n}\right)$ is a harmonic map into $N$ satisfying $\left.u\right|_{\Gamma_{\mathrm{D}}}=u_{\mathrm{D}}$.

The sequence $\left(u_{h}\right)_{h>0}$ can be constructed with Algorithm A, and Theorem I implies that it satisfies the conditions of Theorem II provided that $\left\|\nabla u_{h}^{0}\right\| \leq C$ for all $h>0$. This can be guaranteed if there exists a continuous, piecewise smooth vector field $u^{0}: M \rightarrow N$ with $\left.u^{0}\right|_{\Gamma_{\mathrm{D}}}=u_{\mathrm{D}}$. The asymptotic right-angled condition allows the usage of highly graded, locally refined triangulations but is restrictive 
in the case of nonflat surfaces $M$. It can be shown that the condition is not necessary if the sequence $\left(u_{h}\right)_{h>0}$ is uniformly bounded in $W^{1,2+\sigma}\left(M ; \mathbb{R}^{n}\right)$ for some positive $\sigma$. In view of this and the fact that harmonic maps are smooth if $d=2$, the angle condition appears to be a technical deficiency of the method of proof rather than a necessary condition. A sufficient condition for the existence of a sequence of logarithmically right-angled triangulations is the existence of a sequence of uniformly strictly acute triangulations; cf. Remark 2.2 below. If $N=S^{n-1}$ is the $(n-1)$-dimensional unit sphere, then $M$ does not have to be two-dimensional and any sequence of regular triangulations leads to the assertion of Theorem II; cf. [6]. Note that the existence of a harmonic map into $N$ is implicitly assumed by requiring that there exists a sequence of initial discrete vector fields $\left(u_{h}^{0}\right)_{h>0}$ which is bounded in $W^{1,2}\left(M ; \mathbb{R}^{n}\right)$.

The proof of Theorem II follows ideas from [23, 34] and employs a discrete moving frame to rewrite the discrete Euler-Lagrange equations as an equivalent Hodge system. A discrete Coulomb gauge of the orthonormal frame leads to connection forms that are discrete divergence-free if the underlying triangulation is right-angled. Therefore, on general triangulations, a discrete Hodge (or Helmholtz) decomposition of the connection forms that makes use of nonconforming finite element spaces leads to nonvanishing gradient contributions. To show that corresponding terms in the Hodge system vanish as $h \rightarrow 0$ we require the sequence of triangulations to be logarithmically right-angled. The limit of the remaining part can be, owing to a Jacobian structure, identified with weak concentration and compensation compactness principles based on results in 32 together with the fact that the set of (discrete) harmonic fields on $M$ is finite dimensional.

The outline of this work is as follows. In Section 2 we recall equivalent characterizations of harmonic maps and provide some tools for the analysis of Algorithm A; a proof of Theorem I follows in Section 3. In Section 4 we prove Theorem II in a periodic setting; the reduction of a general setting to the periodic one and hence a full proof of Theorem II will be given in Section [5. Finally, in Section 6 we provide a numerical experiment discussing also modifications of Algorithm A for the case of nonflat domains $M$. A brief review of weak compactness results for harmonic maps is provided in Appendix $\mathrm{A}$, some auxiliary results from measure theory are given in Appendix B

\section{Preliminaries}

Throughout this paper, $M$ denotes a bounded, polygonal or polyhedral Lipschitz domain in $\mathbb{R}^{d}, d=2,3$. The $k$-dimensional submanifold $N \subset \mathbb{R}^{n}$ is assumed to be compact, without boundary, and $C^{2}$ regular.

2.1. Notation. We use standard notation for Sobolev and Lebesgue spaces and write $\|\cdot\|$ whenever $\|\cdot\|_{L^{2}(M)}$ is meant. The vectorial curl of a function $v$, denoted $\operatorname{Curl} v$, is defined as $\operatorname{Curl} v:=\left(-\partial_{2} v, \partial_{1} v\right)^{\mathrm{T}}$ while the scalar curl of a vector field $\psi=\left(\psi_{1}, \psi_{2}\right)$ is defined as curl $\psi:=\partial_{1} \psi_{2}-\partial_{2} \psi_{1}$. The $s$-dimensional Hausdorff measure of a set $A \subset \mathbb{R}^{d}$ is denoted $\mathcal{H}^{s}(A)$. The topological dual of a Banach space $X$ is denoted by $X^{*}$.

2.2. Equivalent characterizations of harmonic maps. The following equivalent characterizations of harmonic maps are important for the analysis of Algorithm A. 
Proposition 2.1 (23]). A vector field $u \in W^{1,2}\left(M ; \mathbb{R}^{n}\right)$ such that $u(x) \in N$ for almost every $x \in M$ is a harmonic map into $N$, i.e., it is stationary for

$$
v \mapsto \frac{1}{2} \int_{M}|\nabla v|^{2} \mathrm{~d} s
$$

with respect to perturbations of the form $\pi_{N}(u+\phi)$ for vector fields $\phi \in L^{\infty}\left(M ; \mathbb{R}^{n}\right) \cap$ $W^{1,2}\left(M ; \mathbb{R}^{n}\right)$ that are compactly supported in $M$, if and only if one of the following equivalent conditions is satisfied:

(i) for all $v \in W_{0}^{1,2}\left(M ; \mathbb{R}^{n}\right)$ such that $v(x) \in T_{u(x)} N$ for almost every $x \in M$ we have

$$
(\nabla u ; \nabla v)=0
$$

(ii) if $\left(e^{i}\right)_{i=1,2, \ldots, k} \subset W^{1,2}\left(M ; \mathbb{R}^{n}\right)$ are such that the vectors $e^{1}(x), e^{2}(x), \ldots, e^{k}(x)$ form an orthonormal basis for $T_{u(x)} N$ for almost every $x \in M$ and if $\vartheta^{i}:=$ $\sum_{\alpha=1}^{n} e^{i, \alpha} \nabla u^{\alpha}$ and $\omega^{i j}:=\sum_{\alpha=1}^{n} e^{j, \alpha} \nabla e^{i, a}$ then we have

$$
\left(\vartheta^{i} ; \nabla \eta\right)+\sum_{j=1}^{k}\left(\omega^{i j} \cdot \vartheta^{j} ; \eta\right)=0
$$

for all $\eta \in W_{0}^{1,2}(M) \cap L^{\infty}(M)$ and $i=1,2, \ldots, k$.

The prerequisites of item (ii) in the proposition are satisfied if $N$ is parallelizable in the sense that there exist continuously differentiable vector fields

$$
e_{1}, e_{2}, \ldots, e_{k}: N \rightarrow \mathbb{R}^{n}
$$

such that for all $p \in N$ the vectors $\left(e_{1}(p), e_{2}(p), \ldots, e_{k}(p)\right)$ form an orthonormal basis for $T_{p} N$. Not every smooth submanifold $N$ is parallelizable; e.g., the unit sphere $S^{2}$ is not. A construction in [26] shows that every compact $C^{4}$ submanifold $N$ without boundary can be isometrically embedded into a parallelizable $C^{3}$ submanifold $\widehat{N}$ and there exists an isometric isomorphism $J: N \rightarrow \widehat{N}$ such that if $u: M \rightarrow N$ is a harmonic map into $N$, then $J \circ u: M \rightarrow \widehat{N}$ is a harmonic map into $\widehat{N}$; cf. [26, Lemma 4.1.2] and [25, 12] for details. For the subsequent analysis it is therefore sufficient to assume that $N$ is $C^{4}$.

2.3. Finite element spaces. Given a regular triangulation $\mathcal{T}_{h}$ of $M$ into triangles or tetrahedra for $d=2$ or $d=3$, respectively, we let $\mathcal{N}_{h} \subset \bar{M}$ denote the set of all nodes in $\mathcal{T}_{h}$ (vertices of elements) and $\mathcal{E}_{h}$ the set of all $(d-1)$-dimensional subsimplices of elements in $\mathcal{T}_{h}$, i.e., edges of triangles if $d=2$ or faces of tetrahedra if $d=3$. We define $h_{K}:=\operatorname{diam}(K)$ for all $K \in \mathcal{T}_{h}$ and set $h:=\max _{K \in \mathcal{T}_{h}} h_{K}$ and $h_{\min }:=\min _{K \in \mathcal{T}_{h}} h_{K}$; we write $h_{E}:=\operatorname{diam}(E)$ for all $E \in \mathcal{E}_{h}$. The lowest order $C^{0}$ conforming finite element space $\mathcal{S}^{1}\left(\mathcal{T}_{h}\right)$ subordinate to the triangulation $\mathcal{T}_{h}$ consists of all globally continuous, $\mathcal{T}_{h}$-elementwise affine functions and the space $\mathcal{L}^{0}\left(\mathcal{T}_{h}\right)$ is the set of $\mathcal{T}_{h}$-elementwise constant functions on $M$, i.e.,

$$
\begin{aligned}
& \mathcal{S}^{1}\left(\mathcal{T}_{h}\right):=\left\{\phi_{h} \in C(\bar{M}):\left.\phi_{h}\right|_{K} \text { affine for all } K \in \mathcal{T}_{h}\right\}, \\
& \mathcal{L}^{0}\left(\mathcal{T}_{h}\right):=\left\{v_{h} \in L^{\infty}(M):\left.v_{h}\right|_{K} \text { constant for all } K \in \mathcal{T}_{h}\right\} .
\end{aligned}
$$

For a subset $\Gamma_{\mathrm{D}} \subseteq \partial M$ which is either empty or satisfies $\mathcal{H}^{d-1}\left(\Gamma_{\mathrm{D}}\right)>0$ we set

$$
\mathcal{S}_{\mathrm{D}}^{1}\left(\mathcal{T}_{h}\right):= \begin{cases}\left\{v_{h} \in \mathcal{S}^{1}\left(\mathcal{T}_{h}\right):\left.v_{h}\right|_{\Gamma_{\mathrm{D}}}=0\right\} & \text { if } \Gamma_{\mathrm{D}} \neq \emptyset \\ \left\{v_{h} \in \mathcal{S}^{1}\left(\mathcal{T}_{h}\right): \int_{M} v_{h} \mathrm{~d} x=0\right\} & \text { if } \Gamma_{\mathrm{D}}=\emptyset\end{cases}
$$


The nodal basis $\left(\varphi_{z}: z \in \mathcal{N}_{h}\right)$ of $\mathcal{S}^{1}\left(\mathcal{T}_{h}\right)$ consists of the hat functions $\varphi_{z} \in \mathcal{S}^{1}\left(\mathcal{T}_{h}\right)$ which satisfy $\varphi_{z}(z)=1$ and $\varphi_{z}\left(z^{\prime}\right)=0$ for all distinct $z, z^{\prime} \in \mathcal{N}_{h}$. We set $\omega_{z}:=$ $\operatorname{supp} \varphi_{z}$ and $h_{z}:=\operatorname{diam}\left(\omega_{z}\right)$ for all $z \in \mathcal{N}_{h}$. For a function $\phi \in C(\bar{M})$ its nodal interpolant $\mathcal{I}_{h} \phi \in \mathcal{S}^{1}\left(\mathcal{T}_{h}\right)$ is defined by

$$
\mathcal{I}_{h} \phi:=\sum_{z \in \mathcal{N}_{h}} \phi(z) \varphi_{z} .
$$

We also define an interpolation operator acting on continuous vector fields on $M$ by applying $\mathcal{I}_{h}$ to each component of the vector field. It is well known that there exists a constant $C>0$ such that for every $\phi \in C(\bar{M})$ with $\left.\phi\right|_{K} \in H^{2}(K)$ for all $K \in \mathcal{T}_{h}$ the approximation error satisfies for each $K \in \mathcal{T}_{h}$,

$$
h_{K}^{-2}\left\|\phi-\mathcal{I}_{h} \phi\right\|_{L^{2}(K)}+h_{K}^{-1}\left\|\nabla\left(\phi-\mathcal{I}_{h} \phi\right)\right\|_{L^{2}(K)} \leq C\left\|D^{2} \phi\right\|_{L^{2}(K)} .
$$

Moreover, $\mathcal{I}_{h}$ is $W^{1, \infty}$ stable; i.e., $\left\|\nabla \mathcal{I}_{h} v\right\|_{L^{\infty}(M)} \leq C\|\nabla v\|_{L^{\infty}(M)}$ for all $v \in$ $W^{1, \infty}(M)$. We say that $\mathcal{T}_{h}$ is weakly acute if the off-diagonal entries of the stiffness matrix are nonpositive, i.e., if

$$
\mathbf{K}_{z z^{\prime}}:=\int_{M} \nabla \varphi_{z} \cdot \nabla \varphi_{z^{\prime}} \mathrm{d} x \leq 0
$$

for all distinct $z, z^{\prime} \in \mathcal{N}_{h}$. If $d=2$, then $\mathcal{T}_{h}$ is weakly acute if and only if every sum of angles opposite to an inner edge is bounded by $\pi$ and every angle opposite to an edge on the boundary is bounded by $\pi / 2$. If $d=3$, then a sufficient condition is that all angles between faces of tetrahedra are bounded by $\pi / 2$. We notice that for $v_{h} \in \mathcal{S}^{1}\left(\mathcal{T}_{h}\right)$ we have, cf. [7],

$$
\left\|\nabla v_{h}\right\|^{2}=-\frac{1}{2} \sum_{z, z^{\prime} \in \mathcal{N}_{h}} \mathbf{K}_{z z^{\prime}}\left|v_{h}(z)-v_{h}\left(z^{\prime}\right)\right|^{2},
$$

where summands with $z=z^{\prime}$ vanish. We will need a discrete version of a product rule. Following [5], we know that there exists a linear operator $\mathbf{A}: \mathcal{S}^{1}\left(\mathcal{T}_{h}\right) \rightarrow$ $\mathcal{L}^{0}\left(\mathcal{T}_{h}\right)^{d \times d}$ such that for all $v_{h}, w_{h} \in \mathcal{S}^{1}\left(\mathcal{T}_{h}\right)$ the identity

$$
\nabla \mathcal{I}_{h}\left[v_{h} w_{h}\right]=\mathbf{A}\left(v_{h}\right) \nabla w_{h}+\mathbf{A}\left(w_{h}\right) \nabla v_{h}
$$

holds almost everywhere on $M$ and for each $K \in \mathcal{T}_{h}$ we have

$$
\left\|\mathbf{A}\left(v_{h}\right)-v_{h} \mathbf{I}_{d \times d}\right\|_{L^{\infty}(K)} \leq C h_{K}\left\|\nabla v_{h}\right\|_{L^{\infty}(K)} .
$$

Moreover, if $d=2$ we have for all $K \in \mathcal{T}_{h}$ that

$$
\left\|\mathbf{A}\left(v_{h}\right)-\mathbf{A}^{\mathrm{T}}\left(v_{h}\right)\right\|_{L^{\infty}(K)} \leq C \min _{\gamma=1,2,3}\left|\cos \alpha_{K, \gamma}\right|\left\|v_{h}\right\|_{L^{\infty}(K)},
$$

where $\alpha_{K, \gamma}, \gamma=1,2, \ldots, d+1$ are the interior angles of the triangle $K$. In particular, $\left.\mathbf{A}\left(v_{h}\right)\right|_{K}$ is symmetric for all $v_{h} \in \mathcal{S}^{1}\left(\mathcal{T}_{h}\right)$ if and only if $K$ has a right angle.

Proof of (6) -(8). Given $K=\operatorname{conv}\left\{z_{0}, z_{1}, \ldots, z_{d}\right\} \in \mathcal{T}_{h}, z_{0}, z_{1}, \ldots, z_{d} \in \mathcal{N}_{h}$, let $h_{E_{\gamma}}:=$ $\left|z_{\gamma}-z_{0}\right|$ and $\widehat{z}_{\gamma}:=h_{E_{\gamma}} x_{\gamma}, \gamma=1,2, \ldots, d$, where $\left(x_{\gamma}\right)_{\gamma=1,2, \ldots, d}$ denotes the canonical basis in $\mathbb{R}^{d}$. Set $\widehat{z}_{0}:=0$ and define $\widehat{K}:=\operatorname{conv}\left\{\widehat{z}_{0}, \widehat{z}_{1}, \ldots, \widehat{z}_{d}\right\}$. Then, for the linear mapping $\mathcal{F}_{K}: \widehat{x} \mapsto z_{0}+\mathbf{G}_{h} \widehat{x}$ that maps the scaled reference element $\widehat{K}$ to $K$ such 
that $\mathcal{F}_{K}\left(\widehat{z}_{\gamma}\right)=z_{\gamma}$ for $\gamma=0,1, \ldots, d$, we set $\widehat{v}_{h}:=v_{h} \circ \mathcal{F}_{K}, \widehat{w}_{h}:=w_{h} \circ \mathcal{F}_{K}$, and $\widehat{\mathcal{I}}_{h}\left[\widehat{v}_{h} \widehat{w}_{h}\right]:=\mathcal{I}_{h}\left[v_{h} w_{h}\right] \circ \mathcal{F}_{K}$. For $\gamma=1,2, \ldots, d$ we have that

$$
\begin{aligned}
\widehat{\partial}_{\gamma} \widehat{\mathcal{I}}_{h}\left[\widehat{v}_{h} \widehat{w}_{h}\right]= & \frac{1}{h_{E_{\gamma}}}\left(\widehat{v}_{h}\left(\widehat{z}_{\gamma}\right) \widehat{w}_{h}\left(\widehat{z}_{\gamma}\right)-\widehat{v}_{h}\left(\widehat{z}_{0}\right) \widehat{w}_{h}\left(\widehat{z}_{0}\right)\right) \\
= & \frac{1}{2 h_{E_{\gamma}}}\left\{\left(\widehat{v}_{h}\left(\widehat{z}_{0}\right)+\widehat{v}_{h}\left(\widehat{z}_{\gamma}\right)\right)\left(\widehat{w}_{h}\left(\widehat{z}_{\gamma}\right)-\widehat{w}_{h}\left(\widehat{z}_{0}\right)\right)\right. \\
& \left.\quad+\left(\widehat{w}_{h}\left(\widehat{z}_{\gamma}\right)+\widehat{w}_{h}\left(\widehat{z}_{0}\right)\right)\left(\widehat{v}_{h}\left(\widehat{z}_{\gamma}\right)-\widehat{v}_{h}\left(\widehat{z}_{0}\right)\right)\right\} \\
=: & \widehat{\mathbf{A}}^{\gamma \gamma}\left(\widehat{v}_{h}\right) \widehat{\partial}_{\gamma} \widehat{w}_{h}+\widehat{\mathbf{A}}^{\gamma \gamma}\left(\widehat{w}_{h}\right) \widehat{\partial}_{\gamma} \widehat{v}_{h} .
\end{aligned}
$$

Since $\nabla v_{h}=\mathbf{G}_{h}^{-\mathrm{T}} \widehat{\nabla} \widehat{v}_{h}$, (6) follows from setting

$$
\mathbf{A}\left(v_{h}\right):=\mathbf{G}_{h}^{-\mathrm{T}} \widehat{\mathbf{A}}\left(\widehat{v}_{h}\right) \mathbf{G}_{h}^{\mathrm{T}} .
$$

For $\gamma=1,2, \ldots, d$ the mapping $x \mapsto \mathbf{A}^{\gamma \gamma}(v)-v(x)$ vanishes at $x=\left(z_{\gamma}+z_{0}\right) / 2$, and thus a discrete Poincaré inequality and $\left|\mathbf{G}_{h}^{-1}\right|,\left|\mathbf{G}_{h}\right| \leq C$ imply (7). Notice that

$$
\mathbf{A}\left(v_{h}\right)-\mathbf{A}^{\mathrm{T}}\left(v_{h}\right)=\left(\mathbf{G}_{h}^{-\mathrm{T}}-\mathbf{G}_{h}\right) \widehat{\mathbf{A}}\left(\widehat{v}_{h}\right) \mathbf{G}_{h}^{\mathrm{T}}+\mathbf{G}_{h} \widehat{\mathbf{A}}\left(\widehat{v}_{h}\right)\left(\mathbf{G}_{h}^{\mathrm{T}}-\mathbf{G}_{h}^{-1}\right)
$$

and

$$
\left|\mathbf{G}_{h}^{-1}-\mathbf{G}_{h}^{\mathrm{T}}\right| \leq C\left|\mathbf{I}_{d \times d}-\mathbf{G}_{h} \mathbf{G}_{h}^{\mathrm{T}}\right| .
$$

The last asserted estimate thus follows from the fact that for $d=2$ we have

$$
\mathbf{G}_{h}=\left[h_{E_{1}}^{-1}\left(z_{1}-z_{0}\right) h_{E_{2}}^{-1}\left(z_{2}-z_{0}\right)\right]
$$

so that

$$
\left|\mathbf{I}_{2 \times 2}-\mathbf{G}_{h} \mathbf{G}_{h}^{\mathrm{T}}\right|=\left|\left[\begin{array}{cc}
0 & \cos \alpha_{K} \\
\cos \alpha_{K} & 0
\end{array}\right]\right| \leq C\left|\cos \alpha_{K}\right|,
$$

where $\alpha_{K}$ is the inner angle of $K$ between the vectors $z_{1}-z_{0}$ and $z_{2}-z_{0}$. Since we can interchange the role of the nodes we verify (8).

For $d=2$ a sequence of triangulations $\left(\mathcal{T}_{h}\right)_{h>0}$ is called logarithmically rightangled if

$$
\lim _{h \rightarrow 0} \log h_{\min }^{-1} \sup _{K \in \mathcal{T}_{h}} \inf _{\gamma=1,2,3}\left|\cos \alpha_{K, \gamma}\right|=0 .
$$

Remark 2.2. A sufficient condition for the existence of a sequence of logarithmically right-angled triangulations is the existence of a sequence of uniformly strictly acute triangulations. A triangulation $\mathcal{T}_{h}$ is called strictly acute if all interior angles of triangles are bounded away from $\pi / 2$. A right-angled refinement $\widehat{\mathcal{T}}_{h}$ of a strictly acute triangulation $\mathcal{T}_{h}$ is obtained by connecting the vertices and midpoints of edges of triangles in $\mathcal{T}_{h}$ with their circumcenters; cf. Figure 1. This construction is also possible when the domain $M$ is curved and newly created nodes are projected onto $M$.

We will frequently employ inverse inequalities, which assert that for $v_{h} \in \mathcal{S}^{1}\left(\mathcal{T}_{h}\right)$ we have, cf., e.g., 13,

$$
\begin{aligned}
\left\|\nabla v_{h}\right\|_{L^{p}(K)} & \leq C h_{K}^{-1}\left\|v_{h}\right\|_{L^{p}(K)}, \\
\left\|v_{h}\right\|_{L^{\infty}(M)} & \leq C h_{\min }^{1-d / 2} \log h_{\min }^{-1}\left\|v_{h}\right\|_{H^{1}(M)}, \\
C^{-1}\left\|v_{h}\right\|_{L^{p}(M)} & \leq\left(\sum_{z \in \mathcal{N}_{h}} h_{z}^{d}\left|v_{h}(z)\right|^{p}\right)^{1 / p} \leq C\left\|v_{h}\right\|_{L^{p}(M)},
\end{aligned}
$$

provided that $h_{\min } \leq 1-C^{\prime}$. 


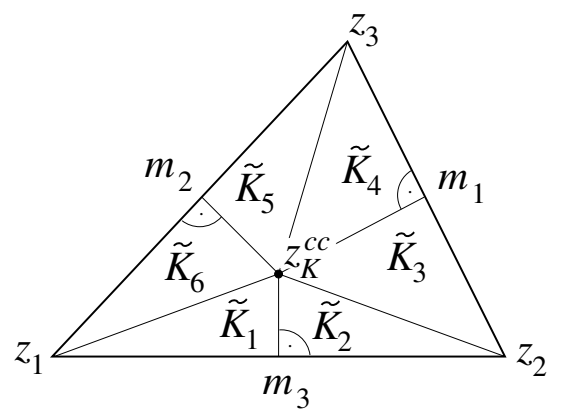

Figure 1. Decomposition of a strictly acute triangle $K$ into rightangled subtriangles $\tilde{K}_{1}, \tilde{K}_{2}, \ldots, \tilde{K}_{6}$ obtained by connecting the vertices $z_{1}, z_{2}, z_{3}$ and midpoints of edges $m_{1}, m_{2}, m_{3}$ with the triangle's circumcenter $z_{K}^{c c}$.

A discrete inner product is for $v, w \in C\left(\bar{M} ; \mathbb{R}^{n}\right)$ defined by

$$
(v ; w)_{h}:=\int_{M} \mathcal{I}_{h}[v \cdot w] \mathrm{d} x=\sum_{z \in \mathcal{N}_{h}} v(z) \cdot w(z) \int_{M} \varphi_{z} \mathrm{~d} x .
$$

Finally, we notice that there exists a constant $C=C(\nu)>0$ such that for all $\mathcal{T}_{h}$-elementwise polynomial functions $\psi_{h} \in C(\bar{M})$ of polynomial degree at most $\nu$ and such that $\psi_{h}(z)=0$ for all $z \in \mathcal{N}_{h}$ we have

$$
\left\|\psi_{h}\right\|_{L^{2}\left(\omega_{z}\right)} \leq C h_{z}\left\|\nabla \psi_{h}\right\|_{L^{2}\left(\omega_{z}\right)} .
$$

2.4. Discrete Helmholtz decomposition on the two-dimensional torus. Throughout this subsection we assume $d=2$ and consider a periodic setting; i.e., we assume

$$
\bar{M}=\mathbb{T}^{2}=\mathbb{R}^{2} / \mathbb{Z}^{2} .
$$

We employ $[0,1]^{2}$ as the fundamental domain of $\mathbb{T}^{2}$. A triangulation $\mathcal{T}_{h}$ of $\mathbb{T}^{2}$ is a regular triangulation of $(0,1)^{2}$ such that for each node $z \in[0,1] \times\{0\} \cup\{0\} \times[0,1]$ there exists a node $z^{\prime} \in[0,1] \times\{1\} \cup\{1\} \times[0,1]$ satisfying $z=z^{\prime}+(1,0)$ or $z=z^{\prime}+(0,1)$. Such two nodes are identified and we set

$\mathcal{S}_{\#}^{1}\left(\mathcal{T}_{h}\right):=\left\{v_{h} \in \mathcal{S}^{1}\left(\mathcal{T}_{h}\right):\left.v_{h}\right|_{(0,1) \times\{0\}}=\left.v_{h}\right|_{(0,1) \times\{1\}}\right.$ and $\left.\left.v_{h}\right|_{\{0\} \times(0,1)}=\left.v_{h}\right|_{\{1\} \times(0,1)}\right\}$.

Two edges $E, E^{\prime} \in \mathcal{E}_{h}$ such that $E=E^{\prime}+(1,0)$ or $E=E^{\prime}+(0,1)$ are identified.

For each edge $E \in \mathcal{E}_{h}$ let $z_{E}$ denote the midpoint of $E$ and define

$$
\begin{aligned}
\mathcal{S}_{\#}^{1, N C}\left(\mathcal{T}_{h}\right):=\left\{v_{h} \in L^{\infty}\left(\mathbb{T}^{2}\right):\left.v_{h}\right|_{K}\right. & \text { is affine for all } K \in \mathcal{T}_{h} \\
& \text { and } \left.v_{h} \text { is continuous at } z_{E} \text { for all } E \in \mathcal{E}_{h}\right\} .
\end{aligned}
$$

The elementwise application of the Curl operator to a function $b_{h} \in \mathcal{S}_{\#}^{1, N C}\left(\mathcal{T}_{h}\right)$ is denoted by $\operatorname{Curl}_{\mathcal{T}_{h}} b_{h}$; i.e., for each $K \in \mathcal{T}_{h}$ we have $\left.\left(\operatorname{Curl}_{\mathcal{T}_{h}} b_{h}\right)\right|_{K}=\left.\operatorname{Curl} b_{h}\right|_{K}$. Given $a_{h} \in \mathcal{S}_{\#}^{1}\left(\mathcal{T}_{h}\right)$ and $b_{h} \in \mathcal{S}_{\#}^{1, N C}\left(\mathcal{T}_{h}\right)$ an elementwise integration by parts reveals that

$$
\left(\nabla a_{h} ; \operatorname{Curl}_{\mathcal{T}_{h}} b_{h}\right)=0
$$


The space of discrete harmonic fields is defined by

$$
\begin{array}{r}
\mathcal{H}_{\#}\left(\mathcal{T}_{h} ; \mathbb{R}^{2}\right):=\left\{H_{h} \in \mathcal{L}^{0}\left(\mathcal{T}_{h}\right)^{2}:\left(H_{h} ; \operatorname{Curl}_{\mathcal{T}_{h}} w_{h}\right)=0 \text { for all } w_{h} \in \mathcal{S}_{\#}^{1, N C}\left(\mathcal{T}_{h}\right)\right. \\
\text { and } \left.\left(H_{h} ; \nabla v_{h}\right)=0 \text { for all } v_{h} \in \mathcal{S}_{\#}^{1}\left(\mathcal{T}_{h}\right)\right\} .
\end{array}
$$

It is straightforward to verify that

$$
\mathcal{H}_{\#}\left(\mathcal{T}_{h} ; \mathbb{R}^{2}\right)=\operatorname{span}\left\{H_{h} \in \mathcal{L}^{0}\left(\mathcal{T}_{h}\right)^{2}: H_{h} \text { is constant on } M\right\} .
$$

The following proposition provides an $L^{2}$ orthogonal decomposition of two-dimensional, $\mathcal{T}_{h}$-elementwise constant vector fields.

Proposition 2.3. Let $\omega_{h} \in \mathcal{L}^{0}\left(\mathcal{T}_{h}\right)^{2}$. There exist uniquely defined $a_{h} \in \mathcal{S}_{\#}^{1}\left(\mathcal{T}_{h}\right)$, $b_{h} \in \mathcal{S}_{\#}^{1, N C}\left(\mathcal{T}_{h}\right)$, and $H_{h} \in \mathcal{H}_{\#}\left(\mathcal{T}_{h} ; \mathbb{R}^{2}\right)$ such that $\int_{M} a_{h} \mathrm{~d} x=0, \int_{M} b_{h} \mathrm{~d} x=0$, and

$$
\omega_{h}=\nabla a_{h}+\operatorname{Curl}_{\mathcal{T}_{h}} b_{h}+H_{h} .
$$

Moreover,

$$
\left\|\omega_{h}\right\|^{2}=\left\|\nabla a_{h}\right\|^{2}+\left\|\operatorname{Curl}_{\mathcal{T}_{h}} b_{h}\right\|^{2}+\left\|H_{h}\right\|^{2} .
$$

Proof. Let $a_{h} \in \mathcal{S}_{\#}^{1}\left(\mathcal{T}_{h}\right)$ be the uniquely defined function that satisfies $\int_{M} a_{h} \mathrm{~d} x=0$ and

$$
\left(\nabla a_{h} ; \nabla v_{h}\right)=\left(\omega_{h} ; \nabla v_{h}\right)
$$

for all $v_{h} \in \mathcal{S}_{\#}^{1}\left(\mathcal{T}_{h}\right)$. Let $b_{h}$ be the uniquely defined function in $\mathcal{S}_{\#}^{1, N C}\left(\mathcal{T}_{h}\right)$ such that $\int_{M} b_{h} \mathrm{~d} x=0$ and

$$
\left(\operatorname{Curl}_{\mathcal{T}_{h}} b_{h} ; \operatorname{Curl}_{\mathcal{T}_{h}} w_{h}\right)=\left(\omega_{h} ; \operatorname{Curl}_{\mathcal{T}_{h}} w_{h}\right)
$$

for all $w_{h} \in \mathcal{S}_{\#}^{1, N C}\left(\mathcal{T}_{h}\right)$. Define $H_{h}:=\omega_{h}-\nabla a_{h}-\operatorname{Curl}_{\mathcal{T}_{h}} b_{h}$. Then, using (14) and the definitions of $a_{h}$ and $b_{h}$ we find that $H_{h} \in \mathcal{H}_{\#}\left(\mathcal{T}_{h} ; \mathbb{R}^{2}\right)$. The orthogonality of the decomposition is an immediate consequence of the construction.

A Clément type operator $\mathcal{A}_{h}: L^{1}\left(\mathbb{T}^{2}\right) \rightarrow \mathcal{S}_{\#}^{1}\left(\mathcal{T}_{h}\right)$ is for $f \in L^{1}\left(\mathbb{T}^{2}\right)$ defined by

$$
\mathcal{A}_{h} f:=\sum_{z \in \mathcal{N}_{h}} f_{z} \varphi_{z}, \quad f_{z}:=\mathcal{H}^{2}\left(\omega_{z}\right)^{-1} \int_{\omega_{z}} f \mathrm{~d} x
$$

where $\omega_{z}=\operatorname{supp} \varphi_{z}$ for all $z \in \mathcal{N}_{h}$. The operator allows us to approximate a function $f \in L^{1}\left(\mathbb{T}^{2}\right)$ by a continuous function $\mathcal{A}_{h} f \in \mathcal{S}_{\#}^{1}\left(\mathcal{T}_{h}\right)$ such that for all $w_{h} \in \mathcal{S}_{\#}^{1, N C}\left(\mathcal{T}_{h}\right)$ we have

$$
\left\|w_{h}-\mathcal{A}_{h} w_{h}\right\|_{L^{2}\left(\omega_{z}\right)} \leq C h_{z}\left\|\operatorname{Curl}_{\mathcal{T}_{h}} w_{h}\right\|_{L^{2}\left(\widehat{\omega}_{z}\right)}
$$

for all $z \in \mathcal{N}_{h}$ and $\widehat{\omega}_{z}:=\bigcup_{y \in \mathcal{N}_{h} \cap \bar{\omega}_{z}} \omega_{y}$, and

$$
\left\|h_{\mathcal{T}_{h}}^{-1}\left(w_{h}-\mathcal{A}_{h} w_{h}\right)\right\| \leq C\left\|\operatorname{Curl}_{\mathcal{T}_{h}} w_{h}\right\|,
$$

where $h_{\mathcal{T}_{h}} \in L^{\infty}\left(\mathbb{T}^{2}\right)$ satisfies $\left.h_{\mathcal{T}_{h}}\right|_{K}=h_{K}$ for all $K \in \mathcal{T}_{h}$. We refer the reader to [15] for proofs. 
2.5. Projections onto surfaces and elementary differential geometry. For a convex set $\mathcal{C} \subset \mathbb{R}^{n}$ it is well known that the orthogonal projection onto $\mathcal{C}$ is well defined in the entire space $\mathbb{R}^{n}$ and Lipschitz continuous with constant less than or equal to 1 . In particular, if $N=\partial \mathcal{C}$, then the projection defines an operator $\pi_{N}: \mathbb{R}^{n} \backslash$ $\mathcal{C} \rightarrow N$. If $N$ is not the boundary of a convex set, then it is still possible to define the nearest-neighbour projection $\pi_{N}$ in the tubular neighbourhood $U_{\delta_{N}}(N):=\{q \in$ $\left.\mathbb{R}^{n}: \operatorname{dist}(q, N)<\delta_{N}\right\}$ of $N$ for some $\delta_{N}>0$ provided that $N$ is $C^{2} ; \pi_{N}$ obeys the identity

$$
\left|q-\pi_{N}(q)\right|=\operatorname{dist}(q, N)
$$

for all $q \in U_{\delta_{N}}(N)$. If $N$ is $C^{\ell}$ for some $\ell \geq 2$, then the mapping $\pi_{N}: U_{\delta}(N) \rightarrow N$ is $C^{\ell-1}$, satisfies $\left.D \pi_{N}(p)\right|_{T_{p} N}=\operatorname{id}_{T_{p} N}$ for all $p \in N$, and $D \pi_{N}(p) \nu=0$ for $\nu \in \mathbb{R}^{n}$ such that $\nu \perp T_{p} N$. It can be shown that $\delta_{N}$ satisfies

$$
\delta_{N}=C_{N}\left(\max _{i=1,2, \ldots, k} \max _{p \in N}\left|\kappa_{i}(p)\right|\right)^{-1},
$$

where $\kappa_{i}(p), i=1,2, \ldots, k$, denote the principal curvatures of $N$ at $p$ and $C_{N}$ is a constant that depends on the global properties of $N$.

It is straightforward to show that there exists $\omega_{N}>0$ such that for all $p \in N$ and all $\tau \in T_{p} N, s \in \mathbb{R}$ with $|\tau| \leq 1$ and $|s| \leq \omega_{N}$, where up to a constant $\omega_{N}$ equals $\delta_{N}$, we have

$$
p+s \tau \in U_{\delta_{N}}(N) .
$$

If $N=\partial \mathcal{C}$ for a convex set $\mathcal{C} \subset \mathbb{R}^{n}$, then $\pi_{N}(p+s \tau)$ is well defined for all $s \in \mathbb{R}$ and we set $\omega_{N}:=\infty$.

We finally notice that there exists a constant $C>0$ such that for all $p, q \in N$ and $\nu \in \mathbb{R}^{n}$ satisfying $\nu \perp T_{p} N$ and $|\nu|=1$ we have

$$
(p-q) \cdot \nu \leq C|p-q|^{2} .
$$

2.6. Weak limits of discrete vector fields into surfaces. The following lemma shows that if a given sequence of finite element functions $\left(u_{h}\right)_{h>0}$ attains its nodal values in a surface and if the sequence converges weakly in $W^{1,2}$, then also the weak accumulation points of the sequence attain their values in the surface almost everywhere.

Lemma 2.4. Suppose that $\left(u_{h}\right)_{h>0}$ is a bounded sequence in $W^{1,2}\left(M ; \mathbb{R}^{n}\right)$ such that for each $h>0$ we have $u_{h} \in \mathcal{S}^{1}\left(\mathcal{T}_{h}\right)^{n}$ and $u_{h}(z) \in N$ for all $z \in \mathcal{N}_{h}$. Then, every weak accumulation point $u \in W^{1,2}\left(M ; \mathbb{R}^{n}\right)$ of the sequence satisfies $u(x) \in N$ for almost every $x \in M$.

Proof. For almost every $x \in M$ set $f_{h}(x):=\operatorname{dist}\left(u_{h}(x), N\right)$. Then, $f_{h}(z)=0$ for all $z \in \mathcal{N}_{h}$ and for every $K \in \mathcal{T}_{h}, x \in K$, and $z \in \mathcal{N}_{h} \cap K$ we have

$$
f_{h}(x)=\int_{0}^{1} \frac{d}{d s} f_{h}(z+s(x-z)) \mathrm{d} s=\int_{0}^{1} \nabla f_{h}(z+s(x-z)) \cdot(x-z) \mathrm{d} s .
$$

Therefore,

and hence

$$
\left|f_{h}(x)\right| \leq C h_{K}\|\nabla \operatorname{dist}(\cdot, N)\|_{L^{\infty}\left(\mathbb{R}^{n}\right)}\left|\nabla u_{h}\right|_{K} \mid
$$

$$
\left\|f_{h}\right\| \leq C h\left\|\nabla u_{h}\right\|
$$

which implies $f_{h}(x) \rightarrow 0$ for almost every $x \in M$. Since a subsequence of $\left(u_{h}\right)_{h>0}$ converges weakly in $W^{1,2}\left(M ; \mathbb{R}^{n}\right)$, hence strongly in $L^{2}\left(M ; \mathbb{R}^{n}\right)$, and in particular 
pointwise almost everywhere, we have by continuity of the distance function that $f_{h}(x)=\operatorname{dist}\left(u_{h}(x), N\right) \rightarrow \operatorname{dist}(u(x), N)$ for almost every $x \in M$. This implies that $u(x) \in N$ for almost every $x \in M$.

The next lemma states that if $u_{h} \in \mathcal{S}^{1}\left(\mathcal{T}_{h}\right)^{n}$ satisfies $u_{h}(z) \in N$ for all $z \in \mathcal{N}_{h}$, then the partial derivatives of $u_{h}$ are almost tangent vectors to $N$.

Lemma 2.5. Let $u_{h} \in \mathcal{S}^{1}\left(\mathcal{T}_{h}\right)^{n}$ be such that $u_{h}(z) \in N$ for all $z \in \mathcal{N}_{h}$. For all $z \in \mathcal{N}_{h}, K \in \mathcal{T}_{h}$, and $\nu \in \mathbb{R}^{n}$ such that $z \in K, \nu \perp T_{u_{h}(z)} N$, and $|\nu|=1$ we have

$$
\left.\partial_{\gamma} u_{h}\right|_{K} \cdot \nu \leq\left. C h_{K}\left|\nabla u_{h}\right|_{K}\right|^{2} \text {. }
$$

Proof. With $\mathcal{F}_{K}$ as in the proof of (6) -(8) in Subsection 2.3, set $\widehat{u}_{h}:=u_{h} \circ \mathcal{F}_{K}$. For $\alpha=1,2, \ldots, n$ we have that $\nabla u_{h}^{\alpha}=\mathbf{G}_{h}^{-\mathrm{T}} \widehat{\nabla} \widehat{u}_{h}^{\alpha}$ and, if $x_{\gamma} \in \mathbb{R}^{d}$ denotes the $\gamma$-th canonical basis vector in $\mathbb{R}^{d}$,

$$
\partial_{\gamma} u_{h}^{\alpha}=\nabla u_{h}^{\alpha} \cdot x_{\gamma}=\left(\mathbf{G}_{h}^{-1} x_{\gamma}\right)^{\mathrm{T}} \widehat{\nabla} \widehat{u}_{h}^{\alpha}
$$

This yields that

$$
\partial_{\gamma} u_{h} \cdot \nu=\sum_{\alpha=1}^{n} \partial_{\gamma} u_{h}^{\alpha} \nu^{\alpha}=\left(\mathbf{G}_{h}^{-1} x_{\gamma}\right)^{\mathrm{T}} \sum_{\alpha=1}^{n} \widehat{\nabla} \widehat{u}_{h}^{\alpha} \nu^{\alpha} .
$$

Now, for each component $\widehat{\partial}_{\delta} \widehat{u}_{h}^{\alpha}$ of $\widehat{\nabla} \widehat{u}_{h}^{\alpha}$ for $\delta=1,2, \ldots, d$, we verify that

$$
\sum_{\alpha=1}^{n} \widehat{\partial}_{\delta} \widehat{u}_{h}^{\alpha} \nu^{\alpha}=\sum_{\alpha=1}^{n} h_{E_{\delta}}^{-1}\left(\widehat{u}_{h}^{\alpha}\left(\widehat{z}_{\delta}\right)-\widehat{u}_{h}^{\alpha}\left(\widehat{z}_{0}\right)\right) \nu^{\alpha}=h_{E_{\delta}}^{-1}\left(\widehat{u}_{h}\left(\widehat{z}_{\delta}\right)-\widehat{u}_{h}\left(\widehat{z}_{0}\right)\right) \cdot \nu .
$$

Noting $\nu \perp T_{u_{h}(z)} N$ and using (18) we infer that

$h_{K}^{-1}\left(\widehat{u}_{h}\left(\widehat{z}_{\delta}\right)-\widehat{u}_{h}\left(\widehat{z}_{0}\right)\right) \cdot \nu \leq C h_{E_{\gamma}}^{-1}\left|\widehat{u}_{h}\left(\widehat{z}_{\delta}\right)-\widehat{u}_{h}\left(\widehat{z}_{0}\right)\right|^{2}=C h_{E_{\gamma}}\left|\widehat{\partial}_{\delta} \widehat{u}_{h}\right|^{2} \leq C h_{K}\left|\widehat{\nabla} \widehat{u}_{h}\right|^{2}$.

On combining the previous estimates, using that $\mathbf{G}_{h}^{-1}$ is bounded $h$-independently, and incorporating the identity $\widehat{\nabla} \widehat{u}_{h}=\mathbf{G}_{h}^{\mathrm{T}} \nabla u_{h}$ with a uniformly bounded matrix $\mathbf{G}_{h}$, we verify that

$$
\partial_{\gamma} u_{h} \cdot \nu \leq C h_{K}\left|\nabla u_{h}\right|^{2}
$$

on $K$, which finishes the proof.

\section{ITERATIVE APPROXIMATION OF HARMONIC MAPS}

The following proposition provides equivalent characterizations of discrete harmonic maps. Its proof is straightforward.

Proposition 3.1. A vector field $u_{h} \in \mathcal{S}^{1}\left(\mathcal{T}_{h}\right)^{n}$ is called a discrete harmonic map into $N$ subject to the boundary data $u_{\mathrm{D}, h}$ if $\left.u_{h}\right|_{\Gamma_{\mathrm{D}}}=u_{\mathrm{D}, h}, u_{h}(z) \in N$ for all $z \in \mathcal{N}_{h}$, and $u_{h}$ is stationary for

$$
v_{h} \mapsto \frac{1}{2} \int_{M}\left|\nabla v_{h}\right|^{2} \mathrm{~d} x
$$

among all $v_{h} \in \mathcal{S}^{1}\left(\mathcal{T}_{h}\right)^{n}$ such that $\left.v_{h}\right|_{\Gamma_{\mathrm{D}}}=u_{\mathrm{D}, h}$ and $v_{h}(z) \in N$ for all $z \in \mathcal{N}_{h}$. This is the case if and only if $u_{h}$ satisfies $u_{h}(z) \in N$ for all $z \in \mathcal{N}_{h}$ and

$$
\left(\nabla u_{h} ; \nabla v_{h}\right)=0
$$

for all $v_{h} \in \mathcal{S}_{\mathrm{D}}^{1}\left(\mathcal{T}_{h}\right)^{n}$ such that $v_{h}(z) \in T_{u_{h}(z)} N$ for all $z \in \mathcal{N}_{h}$. If $u_{\mathrm{D}, h}(z) \in N$ for all $z \in \mathcal{N}_{h} \cap \Gamma_{\mathrm{D}}$, then there exists a discrete harmonic map into $N$ subject to the boundary data $u_{\mathrm{D}, h}$. 
Algorithm A introduced above realizes an iterative solution of (19) by a linearization of the constraint $u_{h}(z) \in N$ about an approximation $u_{h}^{i}(z)$. In the following we analyze its feasibility and termination.

3.1. Feasibility. In the case that the target manifold $N$ is not the boundary of a convex set, then mild but dimension-dependent conditions on the damping parameter $\kappa$ ensure that the projection of the update onto $N$ in Step (4) is well defined in each iteration.

Lemma 3.2. Given any $u_{h} \in \mathcal{S}^{1}\left(\mathcal{T}_{h}\right)^{n}$ satisfying $u_{h}(z) \in N$ for all $z \in \mathcal{N}_{h} \backslash \Gamma_{\mathrm{D}}$, there exists a unique $w_{h} \in \mathcal{S}_{\mathrm{D}}^{1}\left(\mathcal{T}_{h}\right)^{n}$ such that $w_{h}(z) \in T_{u_{h}(z)} N$ for all $z \in \mathcal{N}_{h}$ and

$$
\left(\nabla w_{h} ; \nabla v_{h}\right)=-\left(\nabla u_{h} ; \nabla v_{h}\right)
$$

for all $v_{h} \in \mathcal{S}_{\mathrm{D}}^{1}\left(\mathcal{T}_{h}\right)^{n}$ such that $v_{h}(z) \in T_{u_{h}(z)} N$ for all $z \in \mathcal{N}_{h}$. There exists a constant $C_{N, \mathcal{T}_{h}}>0$ such that the function $w_{h}$ satisfies

$$
\left\|w_{h}\right\|_{L^{\infty}(M)} \leq C_{N, \mathcal{T}_{h}} h_{\min }^{1-d / 2} \log h_{\text {min }}^{-1}\left\|\nabla u_{h}\right\| .
$$

In particular, if $\kappa \leq \omega_{N}\left(C_{0} C_{N, \mathcal{T}_{h}}\right)^{-1} h_{\min }^{d / 2-1} \log h_{\min }^{-1}$ for $C_{0}:=\left\|\nabla u_{h}\right\|$, then

$$
\operatorname{dist}\left(u_{h}(z)+\kappa w_{h}(z), N\right) \leq \delta_{N}
$$

so that $\pi_{N}\left(u_{h}(z)+\kappa w_{h}(z)\right)$ is well defined for all $z \in \mathcal{N}_{h}$.

Remark 3.3. Recall from Subsection 2.5 that $\omega_{N}=\infty$ if $N=\partial \mathcal{C}$ for a convex set $\mathcal{C} \subseteq \mathbb{R}^{n}$ so that in this case the projection in Step 4 is always well defined.

Proof. The set

$$
L_{h}:=\left\{v_{h} \in \mathcal{S}_{\mathrm{D}}^{1}\left(\mathcal{T}_{h}\right)^{n}: v_{h}(z) \in T_{u_{h}(z)} N \text { for all } z \in \mathcal{N}_{h}\right\}
$$

is a subspace of $\mathcal{S}_{\mathrm{D}}^{1}\left(\mathcal{T}_{h}\right)^{n}$. The Lax-Milgram lemma guarantees the existence of a unique $w_{h} \in L_{h}$ such that

$$
\left(\nabla w_{h} ; \nabla v_{h}\right)=-\left(\nabla u_{h} ; \nabla v_{h}\right)
$$

for all $v_{h} \in L_{h}$ and such that $\left\|\nabla w_{h}\right\| \leq\left\|\nabla u_{h}\right\|$. A Poincaré inequality and (10) show that

$$
\left\|w_{h}\right\|_{L^{\infty}(M)} \leq C h_{\min }^{1-d / 2} \log h_{\min }^{-1}\left\|\nabla w_{h}\right\| .
$$

If $\kappa \leq \omega_{N}\left(C_{0} C\right)^{-1} h_{\text {min }}^{d / 2-1} \log h_{\text {min }}^{-1}$, then we have by definition of $\omega_{N}$ in (17) for all $z \in \mathcal{N}_{h}$ that

$$
\operatorname{dist}\left(u_{h}(z)+\kappa w_{h}(z), N\right) \leq \delta_{N},
$$

which ensures that $\pi_{N}\left(u_{h}(z)+\kappa w_{h}(z)\right)$ is well defined for all $z \in \mathcal{N}_{h}$.

3.2. Stability and termination. Slightly more restrictive conditions on the damping parameter $\kappa$ and regularity of $N$ are required to ensure uniform boundedness of iterates of Algorithm $\mathrm{A}$ in $W^{1,2}\left(M ; \mathbb{R}^{n}\right)$. The inductive argument in the proof of the following theorem is adopted from [4.

Theorem 3.4. Suppose that $N$ is $C^{3}$. There exist $C^{\prime}, C^{\prime \prime}>0$ such that if $\kappa \leq$ $C^{\prime} h_{\min }$, then we have for $J \in \mathbb{N}$ and iterates $u_{h}^{0}, u_{h}^{1}, \ldots, u_{h}^{J+1}$ and $w_{h}^{1}, w_{h}^{2}, \ldots, w_{h}^{J+1}$ of Algorithm A that

$$
\left(1-C^{\prime \prime} \kappa h_{\min }^{-1}\right) \kappa \sum_{i=0}^{J}\left\|\nabla w_{h}^{i}\right\|^{2}+\frac{1}{2}\left\|\nabla u_{h}^{J+1}\right\|^{2} \leq \frac{1}{2}\left\|\nabla u_{h}^{0}\right\|^{2} .
$$


The constants $C^{\prime}, C^{\prime \prime}>0$ only depend on $C_{0}:=\left\|\nabla u_{h}^{0}\right\|, N$, and the geometry of $\mathcal{T}_{h}$. In particular, if $\kappa<h_{\min } / C^{\prime \prime}$ and $\varepsilon>0$, then Algorithm $A$ terminates within a finite number of iterations and the output $u_{h}^{*}$ satisfies $u_{h}^{*}(z) \in N$ for all $z \in \mathcal{N}_{h}$, $\left.u_{h}^{*}\right|_{\Gamma_{\mathrm{D}}}=u_{\mathrm{D}, h}$, and

$$
\left|\left(\nabla u_{h}^{*} ; \nabla v_{h}\right)\right| \leq \varepsilon\left\|\nabla v_{h}\right\|
$$

for all $v_{h} \in \mathcal{S}_{\mathrm{D}}^{1}\left(\mathcal{T}_{h}\right)^{n}$ such that $v_{h}(z) \in T_{u_{h}^{*}(z)} N$ for all $z \in \mathcal{N}_{h}$.

Proof. Owing to Lemma 3.2 and the assumptions on $\kappa$ we have that all steps of Algorithm A are well defined. Using that $\pi_{N}$ is twice continuously differentiable in a neighbourhood of $N$, recalling that $\left.D \pi_{N}(p)\right|_{T_{p} N}=\left.\mathrm{id}\right|_{T_{p} N}$ for all $p \in N$, and employing the fact that $N$ is compact so that $\left|u_{h}^{i}(z)\right| \leq C$ for all $z \in \mathcal{N}_{h}$, we verify that the identity

$$
\begin{aligned}
u_{h}^{i+1}(z) & =\pi_{N}\left(u_{h}^{i}(z)+\kappa w_{h}^{i}(z)\right) \\
& =\pi_{N}\left(u_{h}^{i}(z)\right)+\kappa D \pi_{N}\left(u_{h}^{i}(z)\right) w_{h}^{i}(z)+\mathcal{O}\left(\left|\kappa w_{h}^{i}(z)\right|^{2}\right) \\
& =u_{h}^{i}(z)+\kappa w_{h}^{i}(z)+\mathcal{O}\left(\left|\kappa w_{h}^{i}(z)\right|^{2}\right)
\end{aligned}
$$

is satisfied for all $z \in \mathcal{N}_{h}$. We define $r_{h}^{i+1}:=\left(u_{h}^{i+1}-u_{h}^{i}\right)-\kappa w_{h}^{i}$ and deduce from the last estimate that

$$
\left|r_{h}^{i+1}(z)\right| \leq C \kappa^{2}\left|w_{h}^{i}(z)\right|^{2},
$$

with a constant that only depends on $N$. From this estimate and (11) we derive the bound

$$
\left\|r_{h}^{i+1}\right\|^{2} \leq C \kappa^{4}\left\|w_{h}^{i}\right\|_{L^{4}(M)}^{4} .
$$

Owing to the first equation of Algorithm A we have, upon choosing $v_{h}=w_{h}^{i}=$ $\kappa^{-1}\left(u_{h}^{i+1}-u_{h}^{i}\right)-\kappa^{-1} r_{h}^{i+1}$ and employing the binomial identity $b(b-a)=(b-$ $a)^{2} / 2+\left(b^{2}-a^{2}\right) / 2$,

$$
\begin{aligned}
& \left\|\nabla w_{h}^{i}\right\|^{2}=-\left(\nabla u_{h}^{i} ; \nabla w_{h}^{i}\right) \\
& =-\kappa^{-1}\left(\nabla u_{h}^{i} ; \nabla\left(u_{h}^{i+1}-u_{h}^{i}\right)\right)+\kappa^{-1}\left(\nabla u_{h}^{i} ; \nabla r_{h}^{i+1}\right) \\
& =\kappa^{-1}\left(\nabla\left(u_{h}^{i+1}-u_{h}^{i}\right) ; \nabla\left(u_{h}^{i+1}-u_{h}^{i}\right)\right)-\kappa^{-1}\left(\nabla u_{h}^{i+1} ; \nabla\left(u_{h}^{i+1}-u_{h}^{i}\right)\right) \\
& \quad+\kappa^{-1}\left(\nabla u_{h}^{i} ; \nabla r_{h}^{i+1}\right) \\
& =\kappa^{-1}\left\|\nabla\left(u_{h}^{i+1}-u_{h}^{i}\right)\right\|^{2}-\frac{1}{2 \kappa}\left\|\nabla\left(u_{h}^{i+1}-u_{h}^{i}\right)\right\|^{2}-\frac{1}{2 \kappa}\left(\left\|\nabla u_{h}^{i+1}\right\|^{2}-\left\|\nabla u_{h}^{i}\right\|^{2}\right) \\
& \quad+\kappa^{-1}\left(\nabla u_{h}^{i} ; \nabla r_{h}^{i+1}\right),
\end{aligned}
$$

or equivalently,

$$
\left\|\nabla w_{h}^{i}\right\|^{2}+\frac{1}{2 \kappa}\left(\left\|\nabla u_{h}^{i+1}\right\|^{2}-\left\|\nabla u_{h}^{i}\right\|^{2}\right)=\frac{1}{2 \kappa}\left\|\nabla\left(u_{h}^{i+1}-u_{h}^{i}\right)\right\|^{2}+\kappa^{-1}\left(\nabla u_{h}^{i} ; \nabla r_{h}^{i+1}\right) .
$$

To bound the first term on the right-hand side we note that, according to the definition of $r_{h}^{i+1}$,

$$
\frac{1}{2 \kappa}\left\|\nabla\left(u_{h}^{i+1}-u_{h}^{i}\right)\right\|^{2}=\frac{\kappa}{2}\left\|\nabla\left(u_{h}^{i+1}-u_{h}^{i}\right) / \kappa\right\|^{2} \leq \kappa\left\|\nabla w_{h}^{i}\right\|^{2}+\kappa\left\|\kappa^{-1} \nabla r_{h}^{i+1}\right\|^{2} .
$$


An inverse estimate, the bound (22), and the Sobolev estimate $\left\|w_{h}^{i}\right\|_{L^{4}(M)} \leq$ $C\left\|\nabla w_{h}^{i}\right\|$ show that

$$
\kappa^{-1}\left\|\nabla r_{h}^{i+1}\right\|^{2} \leq C \kappa^{-1} h_{\min }^{-2}\left\|r_{h}^{i+1}\right\|^{2} \leq C \kappa^{-1} h_{\min }^{-2} \kappa^{4}\left\|w_{h}^{i}\right\|_{L^{4}(M)}^{4} \leq C \kappa^{3} h_{\min }^{-2}\left\|\nabla w_{h}^{i}\right\|^{4} .
$$

Suppose that $\left\|\nabla u_{h}^{i}\right\| \leq C_{0}$ (which is, by definition of $C_{0}$, satisfied for $i=0$ ). Then we clearly have $\left\|\nabla w_{h}^{i}\right\| \leq C_{0}$ and hence

$$
\kappa^{-1}\left\|\nabla r_{h}^{i+1}\right\|^{2} \leq C C_{0}^{2} \kappa^{3} h_{\min }^{-2}\left\|\nabla w_{h}^{i}\right\|^{2} .
$$

The previous estimates yield that

$$
\frac{1}{2 \kappa}\left\|\nabla\left(u_{h}^{i+1}-u_{h}^{i}\right)\right\|^{2} \leq\left(1+C C_{0}^{2} \kappa^{2} h_{\min }^{-2}\right) \kappa\left\|\nabla w_{h}^{i}\right\|^{2} .
$$

The second term on the right-hand side of (23) is bounded using $\left\|\nabla u_{h}^{i}\right\| \leq C_{0}$, the inverse estimate (91), and (22) by

$$
\begin{aligned}
\kappa^{-1}\left(\nabla u_{h}^{i} ; \nabla r_{h}^{i+1}\right) & \leq \kappa^{-1}\left\|\nabla u_{h}^{i}\right\|\left\|\nabla r_{h}^{i+1}\right\| \leq \kappa^{-1} C_{0}\left\|\nabla r_{h}^{i+1}\right\| \leq \kappa^{-1} C_{0} h_{\min }^{-1}\left\|r_{h}^{i+1}\right\| \\
& \leq \kappa^{-1} C_{0} h_{\min }^{-1} \kappa^{2}\left\|w_{h}^{i}\right\|_{L^{4}(M)}^{2} \leq \kappa^{-1} C C_{0} h_{\min }^{-1} \kappa^{2}\left\|\nabla w_{h}^{i}\right\|^{2} .
\end{aligned}
$$

The combination of (23) with (24) and (25) implies, upon using $\kappa h_{\min }^{-1} \leq C^{\prime}$, that

$$
\left(1-C \kappa h_{\min }^{-1}\right)\left\|\nabla w_{h}^{i}\right\|^{2}+\frac{1}{2 \kappa}\left(\left\|\nabla u_{h}^{i+1}\right\|^{2}-\left\|\nabla u_{h}^{i}\right\|^{2}\right) \leq 0 .
$$

This estimate shows that $\left\|\nabla u_{h}^{i+1}\right\| \leq C_{0}$ and hence justifies the above assumption that $\left\|\nabla u_{h}^{i}\right\| \leq C_{0}$. Therefore, it implies (20). If the algorithm does not terminate within a finite number of iterations, then we have $\left\|\nabla w_{h}^{i}\right\|>\varepsilon$ for $i=0,1, \ldots, I$ and every $I \in \mathbb{N}$, which contradicts (20).

3.3. A sharp refinement for convex targets. If $N=\partial \mathcal{C}$ is the boundary of a convex set, then Algorithm A is well defined for all choices of $\kappa$. Provided that the underlying triangulation $\mathcal{T}_{h}$ is weakly acute and $\kappa<2$, Algorithm $\mathrm{A}$ is also unconditionally stable, owing to the fact that the projection $\pi_{N}$ is nonexpanding. Notice that the following assertion holds without any regularity assumptions on $N$.

Proposition 3.5. Suppose that $\mathcal{T}_{h}$ is weakly acute, $\kappa<2$, and $N=\partial \mathcal{C}$ for a bounded, open, convex set $\mathcal{C} \subset \mathbb{R}^{n}$. Then, for $J \in \mathbb{N}$ and iterates $u_{h}^{0}, u_{h}^{1}, \ldots, u_{h}^{J+1}$ and $w_{h}^{1}, w_{h}^{2}, \ldots, w_{h}^{J+1}$ of Algorithm A we have

$$
\kappa(1-\kappa / 2) \sum_{i=0}^{J}\left\|\nabla w_{h}^{i}\right\|^{2}+\frac{1}{2}\left\|\nabla u_{h}^{J+1}\right\|^{2} \leq \frac{1}{2}\left\|\nabla u_{h}^{0}\right\|^{2} .
$$

In particular, Algorithm A terminates within a finite number of iterations and $u_{h}^{*}$ satisfies (21).

Proof. Since $\left\|\nabla w_{h}^{i}\right\|^{2}=-\left(\nabla u_{h}^{i} ; \nabla w_{h}^{i}\right)$ we have

$$
\begin{aligned}
\frac{1}{2}\left\|\nabla\left(u_{h}^{i}+\kappa w_{h}^{i}\right)\right\|^{2} & =\frac{1}{2}\left\|\nabla u_{h}^{i}\right\|^{2}+\kappa\left(\nabla u_{h}^{i} ; \nabla w_{h}^{i}\right)+\frac{\kappa^{2}}{2}\left\|\nabla w_{h}^{i}\right\|^{2} \\
& =\frac{1}{2}\left\|\nabla u_{h}^{i}\right\|^{2}-\kappa(1-\kappa / 2)\left\|\nabla w_{h}^{i}\right\|^{2} .
\end{aligned}
$$

Employing the fact that for weakly acute triangulations we have that $\mathbf{K}_{z z^{\prime}} \leq 0$ for distinct $z, z^{\prime} \in \mathcal{N}_{h}$, noting that $u_{h}^{i}(z)+\kappa w_{h}^{i}(z) \notin \mathcal{C}$ for all $z \in \mathcal{N}_{h}$, and recalling 
that the projection $\pi_{N}: \mathbb{R}^{n} \backslash \mathcal{C} \rightarrow N$ is Lipschitz continuous with Lipschitz constant less than or equal to 1 , we infer with (5) that

$$
\begin{aligned}
\left\|\nabla u_{h}^{i+1}\right\|^{2} & =-\frac{1}{2} \sum_{z, z^{\prime} \in \mathcal{N}_{h}} \mathbf{K}_{z z^{\prime}}\left|u_{h}^{i+1}(z)-u_{h}^{i+1}\left(z^{\prime}\right)\right|^{2} \\
& =-\frac{1}{2} \sum_{z, z^{\prime} \in \mathcal{N}_{h}} \mathbf{K}_{z z^{\prime}}\left|\pi_{N}\left(u_{h}^{i}(z)+\kappa w_{h}^{i}(z)\right)-\pi_{N}\left(u_{h}^{i}\left(z^{\prime}\right)+\kappa w_{h}^{i}\left(z^{\prime}\right)\right)\right|^{2} \\
& \leq-\frac{1}{2} \sum_{z, z^{\prime} \in \mathcal{N}_{h}} \mathbf{K}_{z z^{\prime}}\left|\left(u_{h}^{i}(z)+\kappa w_{h}^{i}(z)\right)-\left(u_{h}^{i}\left(z^{\prime}\right)+\kappa w_{h}^{i}\left(z^{\prime}\right)\right)\right|^{2} \\
& =\left\|\nabla\left(u_{h}^{i}+\kappa w_{h}^{i}\right)\right\|^{2},
\end{aligned}
$$

where we have used that summands with $z=z^{\prime}$ vanish trivially. A combination of the estimates implies the asserted bound after summation over $i=0,1,2, \ldots, J$. The statement about termination of the algorithm now follows as in the proof of Theorem 3.4 .

Weak acuteness of a triangulation is not a technical detail to ensure stability of Step 4 in Algorithm A for $N=\partial \mathcal{C}$ and $0<\kappa<2$. The angle condition is sharp in the sense of the following example, which is a refinement of an example from [7.
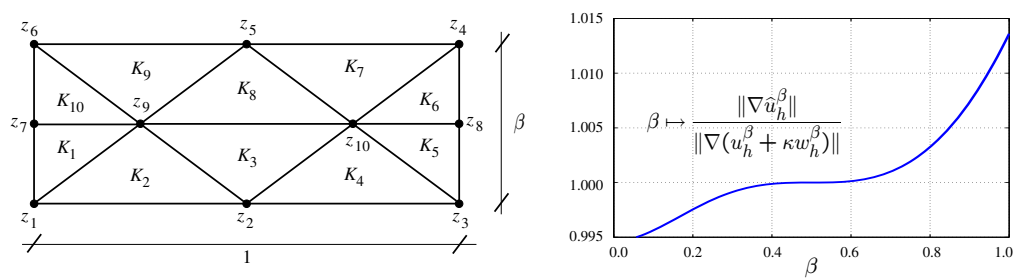

FiguRE 2. Triangulation in Example 3.6 which is weakly acute if and only if $\beta \geq 1 / 2$ (left). $H^{1}$ semi-norms of $u_{h}^{\beta}+\kappa w_{h}^{\beta}$ and its nodal projection $\widehat{u}_{h}^{\beta}$ onto the unit sphere in Example 3.6 (right).

Example 3.6. Let $0<\beta \leq 1, \kappa>0, M:=(0,1) \times(0, \beta)$, and let $\mathcal{T}_{h}$ be the triangulation of $M$ shown in the left plot of Figure 2. There exist $u_{h}^{\beta}, w_{h}^{\beta} \in \mathcal{S}^{1}\left(\mathcal{T}_{h}\right)^{n}$, $n \geq 2$, with $u_{h}^{\beta}(z) \in S^{n-1}$ and $w_{h}^{\beta}(z) \in T_{u_{h}^{\beta}(z)} S^{n-1}$ for all $z \in \mathcal{N}_{h}$ such that for $\widehat{u}_{h}^{\beta} \in \mathcal{S}^{1}\left(\mathcal{T}_{h}\right)^{n}$ defined through $\widehat{u}_{h}^{\beta}(z):=\pi_{S^{n-1}}\left(u_{h}^{\beta}(z)+\kappa w_{h}^{\beta}(z)\right)$ for all $z \in \mathcal{N}_{h}$ we have

$$
\left\|\nabla \widehat{u}_{h}^{\beta}\right\| \leq\left\|\nabla\left(u_{h}^{\beta}+\kappa w_{h}^{\beta}\right)\right\|
$$

if and only if $\mathcal{T}_{h}$ is weakly acute, i.e., if and only if $\beta \geq 1 / 2$.

Proof. Let $u_{h}, w_{h} \in \mathcal{S}^{1}\left(\mathcal{T}_{h}\right)^{n}, n \geq 2$, be the functions satisfying

$$
\begin{gathered}
u_{h}^{\beta}\left(z_{j}\right)=(1,0, \ldots, 0) \quad \text { for } j=1,2, \ldots, 8, \\
u_{h}^{\beta}\left(z_{9}\right)=(-1,0, \ldots, 0), \quad u_{h}^{\beta}\left(z_{10}\right)=(1,0, \ldots, 0),
\end{gathered}
$$

and

$$
w_{h}^{\beta}\left(z_{j}\right)=0 \quad \text { for } j=1,2, \ldots, 9, \quad w_{h}^{\beta}\left(z_{10}\right)=(0, \beta-1 / 2,0, \ldots, 0) / \kappa .
$$

The assertion then follows from straightforward calculations; $\mathrm{cf}$. the right plot of Figure 2. 


\section{WEAK ACCUMULATION OF PERIODIC DISCRETE HARMONIC MAPS IN 2D}

We aim at adapting the weak convergence result for harmonic maps into parallelizable, finite-dimensional manifolds of [23, summarized in Appendix \$ below, to a finite element setting following ideas in 34 for the analysis of a finite difference scheme on planar lattices. The main idea is to establish a discrete version of item (ii) in Proposition 2.1 with a good choice of the orthonormal frame, analyze corresponding perturbation terms, and employ the arguments of 23 to perform the limit passage. For that we assume in this section that $\bar{M}=\mathbb{T}^{2}$ is the twodimensional torus with fundamental domain $[0,1]^{2}$. A reduction of the general case $M \subset \mathbb{R}^{2}$ to the periodic setting will be discussed below in Section 5 Recall that a subscript \# indicates periodicity of discrete functions.

4.1. Discrete Hodge system. We begin with an equivalent characterization of discrete harmonic maps similar to the one given in Proposition 2.1.

Definition 4.1. Let $u_{h} \in \mathcal{S}_{\#}^{1}\left(\mathcal{T}_{h}\right)^{n}$ be such that $u_{h}(z) \in N$ for all $z \in \mathcal{N}_{h}$. Suppose that $\left(e_{h}^{i}\right)_{i=1,2, \ldots, k} \subset \mathcal{S}_{\#}^{1}\left(\mathcal{T}_{h}\right)^{n}$ is an orthonormal frame for

$$
u_{h}^{-1} T N:=\left\{w_{h} \in \mathcal{S}_{\#}^{1}\left(\mathcal{T}_{h}\right)^{n}: w_{h}(z) \in T_{u_{h}(z)} N \text { for all } z \in \mathcal{N}_{h}\right\}
$$

i.e., for all $z \in \mathcal{N}_{h}$ the vectors $e_{h}^{i}(z), i=1,2, \ldots, k$, form an orthonormal basis for $T_{u_{h}(z)} N$. For $i, j=1,2, \ldots, k$ define $\omega_{h}^{i j}, \bar{\omega}_{h}^{i j}, \vartheta_{h}^{i}, \bar{\vartheta}_{h}^{i} \in L^{2}\left(M ; \mathbb{R}^{2}\right)$ by

$$
\omega_{h}^{i j}:=\sum_{\alpha=1}^{n} \mathbf{A}^{\mathrm{T}}\left(e_{h}^{j, \alpha}\right) \nabla e_{h}^{i, \alpha}, \quad \bar{\omega}_{h}^{i j}:=\sum_{\alpha=1}^{n} e_{h}^{j, \alpha} \nabla e_{h}^{i, \alpha},
$$

and

$$
\vartheta_{h}^{i}:=\sum_{\alpha=1}^{n} \mathbf{A}^{\mathrm{T}}\left(e_{h}^{i, \alpha}\right) \nabla u_{h}^{\alpha}, \quad \bar{\vartheta}_{h}^{i}:=\sum_{\alpha=1}^{n} e_{h}^{i, \alpha} \nabla u_{h}^{\alpha},
$$

where $\mathbf{A}$ is defined in (6).

Up to error terms, the characterization of harmonic maps given in Proposition 2.1 holds also in the discrete setting. Notice that we do not assume that $N$ is orientable in the following lemma; continuous unit normals are only required to exist locally.

Lemma 4.2. Suppose that $u_{h} \in \mathcal{S}_{\#}^{1}\left(\mathcal{T}_{h}\right)^{n}$ satisfies $u_{h}(z) \in N$ for all $z \in \mathcal{N}_{h}$ and let $\left(e_{h}^{i}\right)_{i=1,2, \ldots, k} \subset \mathcal{S}_{\#}^{1}\left(\mathcal{T}_{h}\right)^{n}$ be an orthonormal frame for $u_{h}^{-1} T N$. Then, for $i=1,2, \ldots, k$ and all $\eta_{h} \in \mathcal{S}_{\#}^{1}\left(\mathcal{T}_{h}\right)$ we have

$$
\left(\nabla u_{h} ; \nabla \mathcal{I}_{h}\left[\eta_{h} e_{h}^{i}\right]\right)=\sum_{j=1}^{k}\left(\bar{\vartheta}_{h}^{j} \cdot \bar{\omega}_{h}^{i j} ; \eta_{h}\right)+\left(\vartheta_{h}^{i} ; \nabla \eta_{h}\right)+\left[\Lambda_{1}+\Lambda_{2}+\Lambda_{3}\right]\left(u_{h}, e_{h}^{i}, \eta_{h}\right)
$$


where the error terms $\Lambda_{1}, \Lambda_{2}, \Lambda_{3}$ are defined by

$$
\begin{aligned}
& \Lambda_{1}\left(u_{h}, e_{h}^{i}, \eta_{h}\right):=\sum_{\alpha=1}^{n}\left(\nabla u_{h}^{\alpha} ;\left[\mathbf{A}\left(\eta_{h}\right)-\eta_{h} \mathbf{I}\right] \nabla e_{h}^{i, \alpha}\right), \\
& \Lambda_{2}\left(u_{h}, e_{h}^{i}, \eta_{h}\right):=\sum_{\gamma=1}^{2} \sum_{j=1}^{k}\left(\left[\mathcal{I}_{h}\left(e_{h}^{j} \otimes e_{h}^{j}\right)-e_{h}^{j} \otimes e_{h}^{j}\right] \partial_{\gamma} u_{h} ; \eta_{h} \partial_{\gamma} e_{h}^{i}\right), \\
& \Lambda_{3}\left(u_{h}, e_{h}^{i}, \eta_{h}\right):=\sum_{\gamma=1}^{2} \sum_{\ell=k+1}^{n}\left(\mathcal{I}_{h}\left(\nu_{h}^{\ell} \otimes \nu_{h}^{\ell}\right) \partial_{\gamma} u_{h} ; \eta_{h} \partial_{\gamma} e_{h}^{i}\right),
\end{aligned}
$$

and where $\left(\nu_{h}^{\ell}\right)_{\ell=k+1, \ldots, n} \subset \mathcal{S}_{\#}^{1}\left(\mathcal{T}_{h}\right)^{n}$ is such that for all $z \in \mathcal{N}_{h}$ the vectors

$$
e_{h}^{1}(z), \ldots, e_{h}^{k}(z), \nu_{h}^{k+1}(z), \ldots, \nu_{h}^{n}(z)
$$

form an orthonormal basis of $\mathbb{R}^{n}$.

Proof. Fix $1 \leq i \leq k$. Employing (6) we have that

$$
\begin{gathered}
\left(\nabla u_{h} ; \nabla \mathcal{I}_{h}\left[\eta_{h} e_{h}^{i}\right]\right)=\sum_{\alpha=1}^{n}\left(\nabla u_{h}^{\alpha} ; \nabla \mathcal{I}_{h}\left[\eta_{h} e_{h}^{i, \alpha}\right]\right) \\
=\sum_{\alpha=1}^{n}\left(\nabla u_{h}^{\alpha} ; \eta_{h} \nabla e_{h}^{i, \alpha}\right)+\sum_{\alpha=1}^{n}\left(\nabla u_{h}^{\alpha} ;\left[\mathbf{A}\left(\eta_{h}\right)-\eta_{h} \mathbf{I}\right] \nabla e_{h}^{i, \alpha}\right)+\sum_{\alpha=1}^{n}\left(\nabla u_{h}^{\alpha} ; \mathbf{A}\left(e_{h}^{i, \alpha}\right) \nabla \eta_{h}\right) \\
=\sum_{\alpha=1}^{n}\left(\nabla u_{h}^{\alpha} ; \eta_{h} \nabla e_{h}^{i, \alpha}\right)+\Lambda_{1}\left(u_{h}, e_{h}^{i}, \eta_{h}\right)+\left(\vartheta_{h}^{i} ; \nabla \eta_{h}\right) .
\end{gathered}
$$

The choice of the vector fields $\left(e_{h}^{i}\right)_{i=1,2, \ldots, k}$ and $\left(\nu_{h}^{\ell}\right)_{\ell=k+1, \ldots, n}$ and properties of nodal interpolation yield that

$$
\partial_{\gamma} u_{h}=\sum_{j=1}^{k} \mathcal{I}_{h}\left[e_{h}^{j} \otimes e_{h}^{j}\right] \partial_{\gamma} u_{h}+\sum_{\ell=k+1}^{n} \mathcal{I}_{h}\left[\nu_{h}^{\ell} \otimes \nu_{h}^{\ell}\right] \partial_{\gamma} u_{h}
$$

almost everywhere in $M$. Therefore, we deduce that almost everywhere in $M$ we have

$$
\partial_{\gamma} u_{h} \cdot \partial_{\gamma} e_{h}^{i}=\sum_{j=1}^{k}\left(\mathcal{I}_{h}\left[e_{h}^{j} \otimes e_{h}^{j}\right] \partial_{\gamma} u_{h}\right) \cdot \partial_{\gamma} e_{h}^{i}+\sum_{\ell=k+1}^{n}\left(\mathcal{I}_{h}\left[\nu_{h}^{\ell} \otimes \nu_{h}^{\ell}\right] \partial_{\gamma} u_{h}\right) \cdot \partial_{\gamma} e_{h}^{i} .
$$

For each $j=1,2, \ldots, k$ we rewrite the corresponding contribution to the first sum on the right-hand side of the last equation as

$$
\begin{aligned}
\left(\mathcal{I}_{h}\left[e_{h}^{j} \otimes e_{h}^{j}\right] \partial_{\gamma} u_{h}\right) \cdot \partial_{\gamma} e_{h}^{i}= & \left(\left[e_{h}^{j} \otimes e_{h}^{j}\right] \partial_{\gamma} u_{h}\right) \cdot \partial_{\gamma} e_{h}^{i} \\
& +\left(\left\{\mathcal{I}_{h}\left[e_{h}^{j} \otimes e_{h}^{j}\right]-e_{h}^{j} \otimes e_{h}^{j}\right\} \partial_{\gamma} u_{h}\right) \cdot \partial_{\gamma} e_{h}^{i} \\
= & \left(\partial_{\gamma} u_{h} \cdot e_{h}^{j}\right)\left(\partial_{\gamma} e_{h}^{i} \cdot e_{h}^{j}\right) \\
& +\left(\left\{\mathcal{I}_{h}\left[e_{h}^{j} \otimes e_{h}^{j}\right]-e_{h}^{j} \otimes e_{h}^{j}\right\} \partial_{\gamma} u_{h}\right) \cdot \partial_{\gamma} e_{h}^{i} .
\end{aligned}
$$


This implies that

$$
\begin{aligned}
\sum_{\alpha=1}^{n}\left(\nabla u_{h}^{\alpha} ; \eta_{h} \nabla e_{h}^{i, \alpha}\right)= & \sum_{\gamma=1}^{2}\left(\partial_{\gamma} u_{h} ; \eta_{h} \partial_{\gamma} e_{h}^{i}\right) \\
= & \sum_{j=1}^{k} \sum_{\gamma=1}^{2}\left(\partial_{\gamma} u_{h} \cdot e_{h}^{j} ; \eta_{h} \partial_{\gamma} e_{h}^{i} \cdot e_{h}^{j}\right) \\
& +\Lambda_{2}\left(u_{h}, e_{h}^{i}, \eta_{h}\right)+\Lambda_{3}\left(u_{h}, e_{h}^{i}, \eta_{h}\right) \\
= & \sum_{j=1}^{k}\left(e_{h}^{j, \mathrm{~T}} \nabla u_{h} ; \eta_{h} e_{h}^{j, \mathrm{~T}} \nabla e_{h}^{i}\right)+\Lambda_{2}\left(u_{h}, e_{h}^{i}, \eta_{h}\right)+\Lambda_{3}\left(u_{h}, e_{h}^{i}, \eta_{h}\right) \\
= & \sum_{j=1}^{k}\left(\bar{\vartheta}_{h}^{j} \cdot \bar{\omega}_{h}^{i j} ; \eta_{h}\right)+\Lambda_{2}\left(u_{h}, e_{h}^{i}, \eta_{h}\right)+\Lambda_{3}\left(u_{h}, e_{h}^{i}, \eta_{h}\right)
\end{aligned}
$$

and proves the lemma.

4.2. Coulomb gauge for the orthonormal frame. The next lemma shows that an optimal choice of the frame $\left(e_{h}^{i}\right)_{i=1,2, \ldots, k}$ guarantees that $\omega_{h}^{i j}$ is discrete divergence-free for $1 \leq i, j \leq k$ up to perturbations. These perturbations vanish identically if each $K \in \mathcal{T}_{h}$ has a right angle.

Lemma 4.3. Let $\left(e_{h}^{i}\right)_{i=1,2, \ldots, k}$ be minimal for

$$
\left(\widehat{e}_{h}^{i}\right)_{i=1,2, \ldots, k} \mapsto \frac{1}{2} \sum_{i=1}^{k} \int_{\mathbb{T}^{2}}\left|\nabla \widehat{e}_{h}^{i}\right|^{2} \mathrm{~d} x
$$

among all orthonormal frames $\left(\widehat{e}_{h}^{i}\right)_{i=1,2, \ldots, k}$ for $u_{h}^{-1} T N$. Then,

$$
\max _{i=1,2, \ldots, k}\left\|\nabla e_{h}^{i}\right\| \leq C\left(1+\left\|\nabla u_{h}\right\|\right),
$$

and if $\omega_{h}^{i j}$ from Definition 4.1 is defined with such an optimal orthonormal frame, we have for all $\phi_{h} \in \mathcal{S}_{\#}^{1}\left(\mathcal{T}_{h}\right)$ that

$$
\left(\omega_{h}^{i j} ; \nabla \phi_{h}\right)=\Lambda_{4}\left(e_{h}^{i}, e_{h}^{j}, \phi_{h}\right)+\Lambda_{5}\left(e_{h}^{i}, e_{h}^{j}, \phi_{h}\right)
$$

where

$$
\begin{aligned}
\Lambda_{4}\left(e_{h}^{i}, e_{h}^{j}, \phi_{h}\right):=\frac{1}{4} \sum_{\alpha=1}^{n}\{ & \left(\nabla e_{h}^{i, \alpha} ;\left[\mathbf{A}\left(\phi_{h}\right)-\mathbf{A}^{\mathrm{T}}\left(\phi_{h}\right)\right] \nabla e_{h}^{j, \alpha}\right) \\
& \left.-\left(\nabla e_{h}^{j, \alpha} ;\left[\mathbf{A}\left(\phi_{h}\right)-\mathbf{A}^{\mathrm{T}}\left(\phi_{h}\right)\right] \nabla e_{h}^{i, \alpha}\right)\right\},
\end{aligned}
$$

and

$$
\begin{aligned}
\Lambda_{5}\left(e_{h}^{i}, e_{h}^{j}, \phi_{h}\right):=\frac{1}{2} \sum_{\alpha=1}^{n}\{ & \left(\left[\mathbf{A}^{\mathrm{T}}\left(e_{h}^{i, \alpha}\right)-\mathbf{A}\left(e_{h}^{i, \alpha}\right)\right] \nabla e_{h}^{j, \alpha} ; \nabla \phi_{h}\right) \\
& \left.+\left(\left[\mathbf{A}^{\mathrm{T}}\left(e_{h}^{j, \alpha}\right)-\mathbf{A}\left(e_{h}^{j, \alpha}\right)\right] \nabla e_{h}^{i, \alpha} ; \nabla \phi_{h}\right)\right\} .
\end{aligned}
$$

Proof. Let $e_{1}, e_{2}, \ldots, e_{k}: N \rightarrow \mathbb{R}^{n}$ be continuously differentiable vector fields such that for every $p \in N$ the vectors $\left(e_{1}(p), e_{2}(p), \ldots, e_{k}(p)\right)$ form an orthonormal basis 
for $T_{p} N$ and let $\bar{e}_{1}, \bar{e}_{2}, \ldots, \bar{e}_{k}: \mathbb{R}^{n} \rightarrow \mathbb{R}^{n}$ be compactly supported, continuously differentiable vector fields such that $e_{i}(p)=\bar{e}_{i}(p)$ for all $p \in N$ and $1 \leq i \leq k$. Then, the family $\left(\mathcal{I}_{h}\left[\bar{e}^{i} \circ u_{h}\right]\right)_{i=1,2, \ldots, k}$ is an orthonormal frame for $u_{h}^{-1} T \bar{N}$ satisfying

$$
\begin{aligned}
\left\|\nabla \mathcal{I}_{h}\left[\bar{e}^{i} \circ u_{h}\right]\right\|^{2} & =\sum_{K \in \mathcal{T}_{h}} \mathcal{H}^{2}(K)\left\|\nabla \mathcal{I}_{h}\left[\bar{e}^{i} \circ u_{h}\right]\right\|_{L^{\infty}(K)}^{2} \\
& \leq C \sum_{K \in \mathcal{T}_{h}} \mathcal{H}^{2}(K)\left\|\nabla\left[\bar{e}^{i} \circ u_{h}\right]\right\|_{L^{\infty}(K)}^{2} \\
& \leq C\left\|D \bar{e}^{i}\right\|_{L^{\infty}\left(\mathbb{R}^{n}\right)}^{2}\left\|\nabla u_{h}\right\|^{2} \leq C,
\end{aligned}
$$

where we have used $W^{1, \infty}$ stability of $\mathcal{I}_{h}$. Since the optimal frame is minimal among all possible frames, we thus deduce that $\left\|\nabla e_{h}^{i}\right\| \leq C\left(1+\left\|\nabla u_{h}\right\|\right)$ for $1 \leq i \leq k$. Given any $\mathbf{S}_{h} \in \mathcal{S}_{\#}^{1}\left(\mathcal{T}_{h}\right)^{k \times k}$ satisfying $\mathbf{S}_{h}(z) \in S O(k)$, the family $\left(\widetilde{e}_{h}^{i}\right)_{i=1,2, \ldots, k}$ defined by $\widetilde{e}_{h}^{i}:=\mathcal{I}_{h}\left[\sum_{j=1}^{k} \mathbf{S}_{h}^{i j} e_{h}^{j}\right]$ is again an orthonormal frame for $u_{h}^{-1} T N$. Hence, since $\left(e_{h}^{i}\right)_{i=1,2, \ldots, k}$ is minimal, the constant mapping $\mathbf{I}_{k \times k} \in \mathcal{S}_{\#}^{1}\left(\mathcal{T}_{h}\right)^{k \times k}$ is minimal for

$$
\mathbf{S}_{h} \mapsto \frac{1}{2} \sum_{i=1}^{k} \int_{\omega}\left|\nabla \mathcal{I}_{h}\left[\sum_{j=1}^{k} \mathbf{S}_{h}^{i j} \widetilde{e}_{h}^{j}\right]\right|^{2} \mathrm{~d} x
$$

among all $\mathbf{S}_{h} \in \mathcal{S}_{\#}^{1}\left(\mathcal{T}_{h}\right)^{k \times k}$ satisfying $\mathbf{S}_{h}(z) \in S O(k)$ for all $z \in \mathcal{N}_{h}$. Noting that $T_{\mathbf{I}_{k \times k}} S O(k)=s o(k)=\left\{\mathbf{r} \in \mathbb{R}^{k \times k}: \mathbf{r}^{i j}=-\mathbf{r}^{i j}\right.$ for $\left.i, j=1,2, \ldots, k\right\}$, we have for all $\mathbf{r}_{h} \in \mathcal{S}_{\#}^{1}\left(\mathcal{T}_{h}\right)^{k \times k}$ satisfying $\mathbf{r}_{h}(z) \in \operatorname{so}(k)$ for all $z \in \mathcal{N}_{h}$ that

$$
\begin{aligned}
0= & \left.\frac{d}{d \varepsilon}\right|_{\varepsilon=0} \frac{1}{2} \sum_{i=1}^{k} \int_{M}\left|\nabla \mathcal{I}_{h}\left[\sum_{j=1}^{k}\left(\mathbf{I}_{k \times k}^{i j}+\varepsilon \mathbf{r}_{h}^{i j}\right) e_{h}^{j}\right]\right|^{2} \mathrm{~d} x \\
= & \sum_{i=1}^{k}\left(\nabla e_{h}^{i} ; \nabla \mathcal{I}_{h}\left[\sum_{j=1}^{k} \mathbf{r}_{h}^{i j} e_{h}^{j}\right]\right) \\
= & \sum_{i, j=1}^{k} \sum_{\alpha=1}^{n}\left(\nabla e_{h}^{i, \alpha} ; \nabla \mathcal{I}_{h}\left[\mathbf{r}_{h}^{i j} e_{h}^{j, \alpha}\right]\right) \\
= & \sum_{i, j=1}^{k} \sum_{\alpha=1}^{n}\left\{\left(\nabla e_{h}^{i, \alpha} ; \mathbf{A}\left(\mathbf{r}_{h}^{i j}\right) \nabla e_{h}^{j, \alpha}\right)+\left(\nabla e_{h}^{i, \alpha} ; \mathbf{A}\left(e_{h}^{j, \alpha}\right) \nabla \mathbf{r}_{h}^{i j}\right)\right\} \\
= & \sum_{i, j=1}^{k} \sum_{\alpha=1}^{n}\left\{\left(\nabla e_{h}^{i, \alpha} ; \mathbf{A}^{s y m}\left(\mathbf{r}_{h}^{i j}\right) \nabla e_{h}^{j, \alpha}\right)\right. \\
& \left.+\frac{1}{2}\left(\nabla e_{h}^{i, \alpha} ;\left[\mathbf{A}\left(r_{h}^{i j}\right)-\mathbf{A}^{\mathrm{T}}\left(\mathbf{r}_{h}^{i j}\right)\right] \nabla e_{h}^{j, \alpha}\right)+\left(\mathbf{A}^{\mathrm{T}}\left(e_{h}^{j, \alpha}\right) \nabla e_{h}^{i, \alpha} ; \nabla \mathbf{r}_{h}^{i j}\right)\right\}
\end{aligned}
$$

where $\mathbf{A}^{s y m}\left(\mathbf{r}_{h}^{i j}\right):=\left\{\mathbf{A}\left(\mathbf{r}_{h}^{i j}\right)+\mathbf{A}^{\mathrm{T}}\left(\mathbf{r}_{h}^{i j}\right)\right\} / 2$. Upon noting that $\mathbf{r}_{h}$ is skew-symmetric almost everywhere in $M$ and that $\mathbf{A}\left(\mathbf{r}_{h}^{i j}\right)$ depends linearly on $\mathbf{r}_{h}^{i j}$, we infer that

$$
\sum_{i, j=1}^{k} \sum_{\alpha=1}^{n}\left(\nabla e_{h}^{i, \alpha} ; \mathbf{A}^{s y m}\left(\mathbf{r}_{h}^{i j}\right) \nabla e_{h}^{j, \alpha}\right)=0 .
$$


Recalling the definition of $\omega_{h}^{i j}$ we thus deduce that

$$
\sum_{i, j=1}^{k}\left\{\left(\omega_{h}^{i j} ; \nabla \mathbf{r}_{h}^{i j}\right)+\frac{1}{2} \sum_{\alpha=1}^{n}\left(\nabla e_{h}^{i, \alpha} ;\left[\mathbf{A}\left(\mathbf{r}_{h}^{i j}\right)-\mathbf{A}^{\mathrm{T}}\left(\mathbf{r}_{h}^{i j}\right)\right] \nabla e_{h}^{j, \alpha}\right)\right\}=0 .
$$

Given $\phi_{h} \in \mathcal{S}_{\#}^{1}\left(\mathcal{T}_{h}\right)$ and $1 \leq i, j \leq k$ with $i \neq j$, we define $\mathbf{r}_{h} \in \mathcal{S}_{\#}^{1}\left(\mathcal{T}_{h}\right)^{k \times k}$ by setting $\mathbf{r}_{h}^{i j}:=\phi_{h}, \mathbf{r}_{h}^{j i}:=-\phi_{h}$, and $\mathbf{r}_{h}^{i^{\prime} j^{\prime}}:=0$ for $\left(i^{\prime}, j^{\prime}\right) \notin\{(i, j),(j, i)\}$. Then, $\mathbf{r}_{h}(z) \in \operatorname{so}(k)$ for all $z \in \mathcal{N}_{h}$ and (27) yields that

$$
\left(\omega_{h}^{i j} ; \nabla \phi_{h}\right)-\left(\omega_{h}^{j i} ; \nabla \phi_{h}\right)+2 \Lambda_{4}\left(e_{h}^{i}, e_{h}^{j}, \phi_{h}\right)=0,
$$

or equivalently,

$$
\left(\omega_{h}^{i j} ; \nabla \phi_{h}\right)=\Lambda_{4}\left(e_{h}^{i}, e_{h}^{j}, \phi_{h}\right)+\frac{1}{2}\left(\omega_{h}^{j i}+\omega_{h}^{j i} ; \nabla \phi_{h}\right) .
$$

Notice that almost everywhere in $M$ we have that

$$
\begin{aligned}
0= & \nabla \mathcal{I}_{h}\left[e_{h}^{i} \cdot e_{h}^{j}\right]=\nabla \sum_{\alpha=1}^{n} \mathcal{I}_{h}\left[e_{h}^{i, \alpha} e_{h}^{j, \alpha}\right] \\
= & \sum_{\alpha=1}^{n}\left\{\mathbf{A}\left(e_{h}^{i, \alpha}\right) \nabla e_{h}^{j, \alpha}+\mathbf{A}\left(e_{h}^{j, \alpha}\right) \nabla e_{h}^{i, \alpha}\right\} \\
= & \sum_{\alpha=1}^{n}\left\{\mathbf{A}^{\mathrm{T}}\left(e_{h}^{i, \alpha}\right) \nabla e_{h}^{j, \alpha}+\mathbf{A}^{\mathrm{T}}\left(e_{h}^{j, \alpha}\right) \nabla e_{h}^{i, \alpha}\right\} \\
& \quad+\sum_{\alpha=1}^{n}\left\{\left[\mathbf{A}\left(e_{h}^{i, \alpha}\right)-\mathbf{A}^{\mathrm{T}}\left(e_{h}^{i, \alpha}\right)\right] \nabla e_{h}^{j, \alpha}+\left[\mathbf{A}\left(e_{h}^{j, \alpha}\right)-\mathbf{A}^{\mathrm{T}}\left(e_{h}^{j, \alpha}\right)\right] \nabla e_{h}^{i, \alpha}\right\} \\
= & \omega_{h}^{j i}+\omega_{h}^{i j}-\sum_{\alpha=1}^{n}\left\{\left[\mathbf{A}^{\mathrm{T}}\left(e_{h}^{i, \alpha}\right)-\mathbf{A}\left(e_{h}^{i, \alpha}\right)\right] \nabla e_{h}^{j, \alpha}+\left[\mathbf{A}^{\mathrm{T}}\left(e_{h}^{j, \alpha}\right)-\mathbf{A}\left(e_{h}^{j, \alpha}\right)\right] \nabla e_{h}^{i, \alpha}\right\} .
\end{aligned}
$$

The combination of the last two identities implies the lemma.

4.3. Bounds on the error terms. We next incorporate discrete Hodge decompositions of the connection forms $\omega_{h}^{i j}$ in Coulomb gauge and provide bounds on various error terms.

Lemma 4.4. For $1 \leq i, j \leq k$ let $a_{h}^{i j} \in \mathcal{S}_{\#}^{1}\left(\mathcal{T}_{h}\right), b_{h}^{i j} \in \mathcal{S}_{\#}^{1, N C}\left(\mathcal{T}_{h}\right)$, and $H_{h}^{i j} \in$ $\mathcal{H}_{\#}\left(\mathcal{T}_{h} ; \mathbb{R}^{2}\right)$ be the components of the discrete decomposition of $\omega_{h}^{i j}$ according to Proposition 2.3. Define $\widehat{b}_{h}^{i j}:=\mathcal{A}_{h} b_{h}^{i j} \in \mathcal{S}_{\#}^{1}\left(\mathcal{T}_{h}\right)$. Then, for all $\eta_{h} \in \mathcal{S}_{\#}^{1}\left(\mathcal{T}_{h}\right)$ we have

$$
\begin{array}{r}
\left(\nabla u_{h} ; \nabla \mathcal{I}_{h}\left[\eta_{h} e_{h}^{i}\right]\right)=\sum_{j=1}^{k}\left\{\left(\operatorname{Curl} \widehat{b}_{h}^{i j} \cdot \bar{\vartheta}_{h}^{j} ; \eta_{h}\right)+\left(H_{h}^{i j} \cdot \bar{\vartheta}_{h}^{j} ; \eta_{h}\right)\right\}+\left(\vartheta_{h}^{i} ; \nabla \eta_{h}\right) \\
+\Lambda_{1,2,3}\left(u_{h}, e_{h}^{i}, \eta_{h}\right)+\sum_{j=1}^{k} \Lambda_{4,5}\left(e_{h}^{i}, e_{h}^{j}, \psi_{h}^{j}\right) \\
+\sum_{j=1}^{k}\left\{\Theta_{1}\left(u_{h}, e_{h}^{i}, e_{h}^{j}, \eta_{h}\right)+\Theta_{2}\left(u_{h}, e_{h}^{i}, e_{h}^{j}, \eta_{h}\right)\right\},
\end{array}
$$


where $\psi_{h}^{j}:=\mathcal{G}_{h}\left[\eta_{h} \bar{\vartheta}_{h}^{j}\right] \in \mathcal{S}_{\#}^{1}\left(\mathcal{T}_{h}\right)^{n}$ is uniquely defined through $\int_{\mathbb{T}^{2}} \psi_{h}^{j} \mathrm{~d} x=0$ and

$$
\left(\nabla \psi_{h} ; \nabla v_{h}\right)=\left(\bar{\vartheta}_{h}^{j} \eta_{h} ; \nabla v_{h}\right)
$$

for all $v_{h} \in \mathcal{S}_{\#}^{1}\left(\mathcal{T}_{h}\right)^{n}$. We abbreviated $\Lambda_{1,2,3}:=\Lambda_{1}+\Lambda_{2}+\Lambda_{3}$ and $\Lambda_{4,5}:=\Lambda_{4}+\Lambda_{5}$ with the error terms from Lemma 4.2 and Lemma 4.3, respectively, and the terms $\Theta_{1}$ and $\Theta_{2}$ given by

$$
\begin{aligned}
& \Theta_{1}\left(u_{h}, e_{h}^{i}, e_{h}^{j}, \eta_{h}\right):=\left(\left[\bar{\omega}_{h}^{i j}-\omega_{h}^{i j}\right] \cdot \bar{\vartheta}_{h}^{j} ; \eta_{h}\right), \\
& \Theta_{2}\left(u_{h}, e_{h}^{i}, e_{h}^{j}, \eta_{h}\right):=\left(\left[\operatorname{Curl}_{\mathcal{T}_{h}} b_{h}^{i j}-\operatorname{Curl} \widehat{b}_{h}^{i j}\right] \cdot \bar{\vartheta}_{h}^{j} ; \eta_{h}\right) .
\end{aligned}
$$

Proof. Owing to the definitions of $a_{h}^{i j}$ and $\psi_{h}^{j}$ we have

$$
\left(\nabla a_{h}^{i j} \cdot \bar{\vartheta}_{h}^{j} ; \eta_{h}\right)=\left(\nabla a_{h}^{i j} ; \nabla \psi_{h}^{j}\right)=\left(\omega_{h}^{i j} ; \nabla \psi_{h}^{j}\right)=\Lambda_{4,5}\left(e_{h}^{i}, e_{h}^{j}, \psi_{h}^{j}\right) .
$$

According to Lemma 4.2 we thus have

$$
\begin{aligned}
&\left(\nabla u_{h} ; \nabla \mathcal{I}_{h}\left[\eta_{h} e_{h}^{i}\right]\right)= \sum_{j=1}^{k}\left(\bar{\omega}_{h}^{i j} \cdot \bar{\vartheta}_{h}^{j} ; \eta_{h}\right)+\left(\vartheta_{h}^{i} ; \nabla \eta_{h}\right)+\Lambda_{1,2,3}\left(u_{h}, e_{h}^{i}, \eta_{h}\right) \\
&= \sum_{j=1}^{k}\left(\omega_{h}^{i j} \cdot \bar{\vartheta}_{h}^{j} ; \eta_{h}\right)+\left(\vartheta_{h}^{i} ; \nabla \eta_{h}\right)+\Lambda_{1,2,3}\left(u_{h}, e_{h}^{i}, \eta_{h}\right) \\
&+\sum_{j=1}^{k} \Theta_{1}\left(u_{h}, e_{h}^{i}, e_{h}^{j}, \eta_{h}\right) \\
&=\sum_{j=1}^{k}\left\{\left(\operatorname{Curl} \widehat{b}_{h}^{i j} \cdot \bar{\vartheta}_{h}^{j} ; \eta_{h}\right)+\left(H_{h}^{i j} \cdot \bar{\vartheta}_{h}^{j} ; \eta_{h}\right)\right\}+\left(\vartheta_{h}^{i} ; \nabla \eta_{h}\right) \\
&+\Lambda_{1,2,3}\left(u_{h}, e_{h}^{i}, \eta_{h}\right)+\sum_{i, j=1}^{k} \Lambda_{4,5}\left(e_{h}^{i}, e_{h}^{j}, \psi_{h}^{j}\right) \\
&+\sum_{j=1}^{k}\left\{\Theta_{1}\left(u_{h}, e_{h}^{i}, e_{h}^{j}, \eta_{h}\right)+\Theta_{2}\left(u_{h}, e_{h}^{i}, e_{h}^{j}, \eta_{h}\right)\right\}
\end{aligned}
$$

which proves the assertion.

Lemma 4.5. Suppose that there exists $C>0$ such that for all $h>0$ we have $\left\|\nabla u_{h}\right\| \leq C$ and $\max _{i=1,2, \ldots, k}\left\|\nabla e_{h}^{i}\right\| \leq C$. For $1 \leq i \leq k$ there exists $f_{h}^{i} \in \mathcal{S}_{\#}^{1}\left(\mathcal{T}_{h}\right)$ such that for all $\eta_{h} \in \mathcal{S}_{\#}^{1}\left(\mathcal{T}_{h}\right)$ we have

$$
\left(f_{h}^{i} ; \eta_{h}\right)_{h}=\Lambda_{1,2,3}\left(u_{h}, e_{h}^{i}, \eta_{h}\right)+\sum_{j=1}^{k}\left\{\Theta_{1}\left(u_{h}, e_{h}^{i}, e_{h}^{j}, \eta_{h}\right)+\Theta_{2}\left(u_{h}, e_{h}^{i}, e_{h}^{j}, \eta_{h}\right)\right\}
$$

and

$$
\left(f_{h}^{i} ; \eta_{h}\right)_{h} \leq C\left\|\eta_{h}\right\|_{L^{\infty}\left(\mathbb{T}^{2}\right)}
$$

Moreover, for all $\eta_{h} \in \mathcal{S}_{\#}^{1}\left(\mathcal{T}_{h}\right)$ we have

(30) $\left(f_{h}^{i} ; \eta_{h}\right)_{h} \leq C h\left\|\nabla \eta_{h}\right\|_{L^{\infty}\left(\mathbb{T}^{2}\right)}+C \sum_{z \in \mathcal{N}_{h}} h_{z} \max _{j=1,2, \ldots, k}\left\|\nabla e_{h}^{i}\right\|_{L^{\infty}\left(\omega_{z}\right)} \gamma_{h, z, i}^{2}\left|\eta_{h}(z)\right|$, 
where for each $z \in \mathcal{N}_{h}$,

$$
\begin{array}{r}
\gamma_{h, z, i}:=\max \left\{\left\|\nabla u_{h}\right\|_{L^{2}\left(\omega_{z}\right)},\left\|\nabla e_{h}^{1}\right\|_{L^{2}\left(\omega_{z}\right)}, \ldots,\left\|\nabla e_{h}^{k}\right\|_{L^{2}\left(\omega_{z}\right)},\right. \\
\left.\left\|\operatorname{Curl}_{\mathcal{T}_{h}} b_{h}^{i 1}\right\|_{L^{2}\left(\widehat{\omega}_{z}\right)}, \ldots,\left\|\operatorname{Curl}_{\mathcal{T}_{h}} b_{h}^{i k}\right\|_{L^{2}\left(\widehat{\omega}_{z}\right)}\right\} .
\end{array}
$$

Proof. One directly verifies that the right-hand side of (28) defines a linear functional on $\mathcal{S}_{\#}^{1}\left(\mathcal{T}_{h}\right)$ (in $\left.\eta_{h}\right)$. Hence there exists $f_{h}^{i} \in \mathcal{S}_{\#}^{1}\left(\mathcal{T}_{h}\right)$ such that (28) holds for all $\eta_{h} \in \mathcal{S}_{\#}^{1}\left(\mathcal{T}_{h}\right)$. To prove the asserted bounds for $f_{h}^{i}$ we estimate each contribution to the right-hand side of (28) separately. Without further notice we will employ the assumed bounds for $\left\|\nabla u_{h}\right\|$ and $\left\|\nabla e_{h}^{i}\right\|$.

(i) According to the definition and properties of the matrix $\mathbf{A}\left(\eta_{h}\right)$ provided in (6) (8) we have

$$
\Lambda_{1}\left(u_{h}, e_{h}^{i}, \eta_{h}\right) \leq C\left\|\eta_{h}\right\|_{L^{\infty}\left(\mathbb{T}^{2}\right)}
$$

as well as

$$
\Lambda_{1}\left(u_{h}, e_{h}^{i}, \eta_{h}\right) \leq C h\left\|\nabla \eta_{h}\right\|_{L^{\infty}\left(\mathbb{T}^{2}\right)} .
$$

(ii) Since $\left|e_{h}^{j}\right| \leq 1$ almost everywhere in $\mathbb{T}^{2}$ we have

$$
\Lambda_{2}\left(u_{h}, e_{h}^{i}, \eta_{h}\right) \leq C\left\|\eta_{h}\right\|_{L^{\infty}\left(\mathbb{T}^{2}\right)} .
$$

The discrete Poincaré inequality (12) yields $\left\|\mathcal{I}_{h}\left(e_{h}^{j} \otimes e_{h}^{j}\right)-e_{h}^{j} \otimes e_{h}^{j}\right\|_{L^{2}\left(\omega_{z}\right)} \leq$ $C h_{z}\left\|e_{h}^{j}\right\|_{L^{\infty}\left(\omega_{z}\right)}\left\|\nabla e_{h}^{j}\right\|_{L^{2}\left(\omega_{z}\right)}$ so that

$$
\begin{aligned}
\Lambda_{2}\left(u_{h}, e_{h}^{i}, \eta_{h}\right) & =\sum_{z \in \mathcal{N}_{h}} \eta_{h}(z) \sum_{\gamma=1}^{2} \sum_{j=1}^{k}\left(\left[\mathcal{I}_{h}\left(e_{h}^{j} \otimes e_{h}^{j}\right)-e_{h}^{j} \otimes e_{h}^{j}\right] \partial_{\gamma} u_{h} ; \varphi_{z} \partial_{\gamma} e_{h}^{i}\right) \\
& \leq C \sum_{z \in \mathcal{N}_{h}} h_{z}\left\|\nabla e_{h}^{i}\right\|_{L^{\infty}\left(\omega_{z}\right)} \gamma_{h, z, i}^{2}\left|\eta_{h}(z)\right| .
\end{aligned}
$$

(iii) Since $\left|\nu_{h}^{\ell}\right| \leq 1$ almost everywhere in $\mathbb{T}^{2}$ we have

$$
\Lambda_{3}\left(u_{h}, e_{h}^{i}, \eta_{h}\right) \leq C\left\|\eta_{h}\right\|_{L^{\infty}\left(\mathbb{T}^{2}\right)}
$$

Lemma 2.5 implies that $\left|\nu_{h}^{\ell} \cdot \partial_{\gamma} u_{h}\right| \leq C h_{K}\left|\nabla u_{h}\right|^{2}$ on each $K \in \mathcal{T}_{h}$ so that

$$
\Lambda_{3}\left(u_{h}, e_{h}^{i}, \eta_{h}\right) \leq C \sum_{z \in \mathcal{N}_{h}} h_{z}\left\|\nabla e_{h}^{i}\right\|_{L^{\infty}\left(\omega_{z}\right)} \gamma_{h, z, i}^{2}\left|\eta_{h}(z)\right| .
$$

(iv) With the definitions of $\bar{\omega}_{h}^{i j}, \omega_{h}^{i j}$, and $\bar{\vartheta}_{h}^{j}$ and (6) we verify that

$$
\begin{aligned}
\Theta_{1}\left(u_{h}, e_{h}^{i}, e_{h}^{j}, \eta_{h}\right) & =\left(\bar{\omega}_{h}^{i j}-\omega_{h}^{i j} ; \eta_{h} \bar{\vartheta}_{h}^{j}\right) \\
& =\sum_{\alpha=1}^{n}\left(e_{h}^{i, \alpha} \nabla e_{h}^{j, \alpha}-\mathbf{A}^{\mathrm{T}}\left(e_{h}^{i, \alpha}\right) \nabla e_{h}^{j, \alpha} ; \eta_{h} \bar{\vartheta}_{h}^{j}\right) \\
& \leq C\left\|\eta_{h}\right\|_{L^{\infty}\left(\mathbb{T}^{2}\right)} .
\end{aligned}
$$


Incorporating (6) we find that

$$
\begin{aligned}
& \Theta_{1}\left(u_{h}, e_{h}^{i}, e_{h}^{j}, \eta_{h}\right)=\sum_{z \in \mathcal{N}_{h}} \eta_{h}(z)\left(\bar{\omega}_{h}^{i j}-\omega_{h}^{i j} ; \varphi_{z} \bar{\vartheta}_{h}^{j}\right) \\
& \leq C \sum_{z \in \mathcal{N}_{h}} \max _{\alpha=1,2, \ldots, n}\left\|\mathbf{A}\left(e_{h}^{i, \alpha}\right)-e_{h}^{i, \alpha} \mathbf{I}\right\|_{L^{\infty}\left(\omega_{z}\right)}\left\|\nabla e_{h}^{j}\right\|_{L^{2}\left(\omega_{z}\right)}\left\|e_{h}^{j}\right\|_{L^{\infty}\left(\omega_{z}\right)}\left\|\nabla u_{h}\right\|_{L^{2}\left(\omega_{z}\right)}\left|\eta_{h}(z)\right| \\
& \leq C \sum_{z \in \mathcal{N}_{h}} h_{z}\left\|\nabla e_{h}^{i}\right\|_{L^{\infty}\left(\omega_{z}\right)} \gamma_{h, z, i}^{2}\left|\eta_{h}(z)\right| .
\end{aligned}
$$

(v) Owing to inverse estimates, (15), and the definition of $b_{h}^{i j}$ we have that

$$
\left\|\operatorname{Curl}_{\mathcal{T}_{h}} b_{h}^{i j}-\operatorname{Curl} \widehat{b}_{h}^{i j}\right\| \leq C\left\|\operatorname{Curl}_{\mathcal{T}_{h}} b_{h}^{i j}\right\| \leq C\left\|\omega_{h}^{i j}\right\| \leq C .
$$

Therefore, we deduce that

$$
\Theta_{2}\left(u_{h}, e_{h}^{i}, e_{h}^{j}, \eta_{h}\right)=\left(\operatorname{Curl}_{\mathcal{T}_{h}} b_{h}^{i j}-\operatorname{Curl} \widehat{b}_{h}^{i j} ; \eta_{h} \bar{\vartheta}_{h}^{j}\right) \leq C\left\|\eta_{h}\right\|_{L^{\infty}\left(\mathbb{T}^{2}\right)} .
$$

Using that

$$
\operatorname{curl}\left(\eta_{h} \bar{\vartheta}_{h}^{j}\right)=\sum_{\alpha=1}^{n}\left\{\operatorname{Curl} \eta_{h} \cdot\left[e_{h}^{j, \alpha} \nabla u_{h}^{\alpha}\right]+\eta_{h}\left[\operatorname{Curl} e_{h}^{j, \alpha}\right] \cdot \nabla u_{h}^{\alpha}\right\}
$$

we infer with a $\mathcal{T}_{h}$-elementwise integration by parts that

$$
\begin{aligned}
& \Theta_{2}\left(u_{h}, e_{h}^{i}, e_{h}^{j}, \eta_{h}\right)=\left(\operatorname{Curl}_{\mathcal{T}_{h}} b_{h}^{i j}-\operatorname{Curl} \widehat{b}_{h}^{i j} ; \eta_{h} \bar{\vartheta}_{h}^{j}\right) \\
& =-\sum_{\alpha=1}^{n}\left\{\left(b_{h}^{i j}-\widehat{b}_{h}^{i j} ; \operatorname{Curl} \eta_{h} \cdot\left[e_{h}^{j, \alpha} \nabla u_{h}^{\alpha}\right]\right)+\left(b_{h}^{i j}-\widehat{b}_{h}^{i j} ; \eta_{h}\left[\operatorname{Curl} e_{h}^{j, \alpha}\right] \cdot \nabla u_{h}^{\alpha}\right)\right\} \\
& \quad+\sum_{K \in \mathcal{T}_{h}} \int_{\partial K}\left(b_{h}^{i j}-\widehat{b}_{h}^{i j}\right)\left(\eta_{h} \bar{\vartheta}_{h}^{j}\right) \cdot \tau_{K} \mathrm{~d} t,
\end{aligned}
$$

where $\tau_{K}$ is a unit tangent to $\partial K$ for each $K \in \mathcal{T}_{h}$. For the first term on the right-hand side of (31) we have by (16) and with $\left\|\mathrm{Curl} \eta_{h}\right\|_{L^{\infty}\left(\mathbb{T}^{2}\right)}=\left\|\nabla \eta_{h}\right\|_{L^{\infty}\left(\mathbb{T}^{2}\right)}$ that

$$
\begin{aligned}
\sum_{\alpha=1}^{n}\left(b_{h}^{i j}-\right. & \left.\widehat{b}_{h}^{i j} ; \operatorname{Curl} \eta_{h} \cdot\left[e_{h}^{j, \alpha} \nabla u_{h}^{\alpha}\right]\right) \\
& \leq C h\left\|h_{\mathcal{T}_{h}}^{-1}\left(b_{h}^{i j}-\widehat{b}_{h}^{i j}\right)\right\|\left\|\nabla \eta_{h}\right\|_{L^{\infty}\left(\mathbb{T}^{2}\right)}\left\|e_{h}^{j}\right\|_{L^{\infty}\left(\mathbb{T}^{2}\right)}\left\|\nabla u_{h}\right\| \\
& \leq C h\left\|\nabla \eta_{h}\right\|_{L^{\infty}\left(\mathbb{T}^{2}\right)} .
\end{aligned}
$$

The second term on the right-hand side of (31) is estimated by

$$
\begin{gathered}
\sum_{\alpha=1}^{n}\left(b_{h}^{i j}-\widehat{b}_{h}^{i j} ; \eta_{h}\left[\operatorname{Curl} e_{h}^{j, \alpha}\right] \cdot \nabla u_{h}^{\alpha}\right)=\sum_{z \in \mathcal{N}_{h}} \sum_{\alpha=1}^{n} \eta_{h}(z)\left(b_{h}^{i j}-\widehat{b}_{h}^{i j} ; \varphi_{z}\left[\operatorname{Curl} e_{h}^{j, \alpha}\right] \cdot \nabla u_{h}^{\alpha}\right) \\
\leq C \sum_{z \in \mathcal{N}_{h}}\left|\eta_{h}(z)\right|\left\|b_{h}^{i j}-\widehat{b}_{h}^{i j}\right\|_{L^{2}\left(\omega_{z}\right)}\left\|\nabla e_{h}^{j}\right\|_{L^{\infty}\left(\omega_{z}\right)}\left\|\nabla u_{h}\right\|_{L^{2}\left(\omega_{z}\right)} \\
\leq C \sum_{z \in \mathcal{N}_{h}}\left|\eta_{h}(z)\right| h_{z}\left\|\operatorname{Curl}_{\mathcal{T}_{h}} b_{h}^{i j}\right\|_{L^{2}\left(\widehat{\omega}_{z}\right)}\left\|\nabla e_{h}^{j}\right\|_{L^{\infty}\left(\omega_{z}\right)}\left\|\nabla u_{h}\right\|_{L^{2}\left(\omega_{z}\right)} .
\end{gathered}
$$


Notice that the vector field $\eta_{h} \bar{\vartheta}_{h}^{j}=\eta_{h} \sum_{\alpha=1}^{n} e_{h}^{j, \alpha} \nabla u_{h}^{\alpha}$ has continuous tangential components across interelement boundaries and $\widehat{b}_{h}^{i j}$ is continuous so that the boundary contributions to the right-hand side of (31) can be recast as

$$
\sum_{K \in \mathcal{T}_{h}} \int_{\partial K}\left(b_{h}^{i j}-\widehat{b}_{h}^{i j}\right)\left(\eta_{h} \bar{\vartheta}_{h}^{j}\right) \cdot \tau_{K} \mathrm{~d} t=\sum_{E \in \mathcal{E}_{h}} \int_{E}\left[b_{h}^{i j}\right]\left(\eta_{h} \bar{\vartheta}_{h}^{j}\right) \cdot \tau_{E} \mathrm{~d} t .
$$

Since $b_{h}^{i j} \in \mathcal{S}_{\#}^{1, N C}\left(\mathcal{T}_{h}\right)$, the jump $\left.\left[b_{h}^{i j}\right]\right|_{E}$ across $E$ is affine and vanishes at the midpoint of $E$, where $b_{h}^{i j}$ is continuous so that $\int_{E}\left[b_{h}^{i j}\right] \mathrm{d} t=0$. This enables us to subtract an arbitrary constant $c_{E} \in \mathbb{R}^{2}$ from the second factor and to employ a Poincaré inequality on $E$ and discrete trace inequalities to estimate a typical contribution to the right-hand side as

$$
\begin{aligned}
\int_{E}\left[b_{h}^{i j}\right]\left(\eta_{h} \bar{\vartheta}_{h}^{j}\right) \cdot \tau_{E} \mathrm{~d} t & =\int_{E}\left[b_{h}^{i j}\right]\left(\eta_{h} \bar{\vartheta}_{h}^{j}-c_{E}\right) \cdot \tau_{E} \mathrm{~d} t \\
\leq C h_{E} \| \partial\left[b_{h}^{i j}\right] & / \partial t\left\|_{L^{2}(E)} h_{E}^{-1 / 2}\right\| \eta_{h} \bar{\vartheta}_{h}^{j}-c_{E} \|_{L^{2}\left(K_{E}\right)} \\
& \leq C h_{E}^{1 / 2}\left\|\operatorname{Curl}_{\mathcal{T}_{h}} b_{h}^{i j}\right\|_{L^{2}\left(K_{E}\right)} h_{E}^{1 / 2}\left\|\nabla\left(\eta_{h} \bar{\vartheta}_{h}^{j}\right)\right\|_{L^{2}\left(K_{E}\right)}
\end{aligned}
$$

with $K_{E} \in \mathcal{T}_{h}$ such that $E \subset \partial K_{E}$. Noting that $\left|\nabla\left(\eta_{h} \bar{\vartheta}_{h}^{j}\right)\right| \leq\left|\nabla \eta_{h}\right|\left|\bar{\vartheta}_{h}^{j}\right|+$ $\left|\eta_{h}\right|\left|\nabla e_{h}^{j}\right|\left|\nabla u_{h}\right|$, we verify with the above estimates that

$$
\begin{aligned}
& \sum_{K \in \mathcal{T}_{h}} \int_{\partial K}\left(b_{h}^{i j}-\widehat{b}_{h}^{i j}\right)\left(\eta_{h} \bar{\vartheta}_{h}^{j}\right) \cdot \tau_{K} \mathrm{~d} t \\
& \leq C h\left\|\nabla \eta_{h}\right\|_{L^{\infty}\left(\mathbb{T}^{2}\right)}+C \sum_{z \in \mathcal{N}_{h}} h_{z}\left\|\nabla e_{h}^{j}\right\|_{L^{\infty}\left(\omega_{z}\right)}\left\|\operatorname{Curl}_{\mathcal{T}_{h}} b_{h}^{i j}\right\|_{L^{2}\left(\widehat{\omega}_{z}\right)}\left\|\nabla e_{h}^{i}\right\|_{L^{2}\left(\omega_{z}\right)}\left|\eta_{h}(z)\right| .
\end{aligned}
$$

A combination of the estimates derived in (i)-(v) proves the statement.

4.4. Convergence as $h \rightarrow 0$. With the preparations of the previous lemmas, we can investigate convergence behaviour of the different quantities as the maximal mesh-size decays to zero.

Lemma 4.6. Let $\left(\mathcal{T}_{h}\right)_{h>0}$ be a sequence of regular, logarithmically right-angled triangulations. Then, with $\psi_{h}=\mathcal{G}_{h}\left[\eta_{h} \bar{\vartheta}_{h}^{j}\right]$ as in Lemma 4.4 for $\eta_{h} \in \mathcal{S}_{\#}^{1}\left(\mathcal{T}_{h}\right)$ such that $\left\|\eta_{h}\right\|_{L^{\infty}\left(\mathbb{T}^{2}\right)} \leq C$ for all $h>0$, we have

$$
\left|\Lambda_{4}\left(e_{h}^{i}, e_{h}^{j}, \psi_{h}\right)\right|+\left|\Lambda_{5}\left(e_{h}^{i}, e_{h}^{j}, \psi_{h}\right)\right| \rightarrow 0
$$

as $h \rightarrow 0$.

Proof. Owing to the definition of $\Lambda_{4}$ and (8) we have

$$
\begin{aligned}
\Lambda_{4}\left(e_{h}^{i}, e_{h}^{j}, \psi_{h}\right) & \leq C\left\|\nabla e_{h}^{i}\right\|\left\|\nabla e_{h}^{j}\right\|\left\|\mathbf{A}\left(\psi_{h}\right)-\mathbf{A}^{\mathrm{T}}\left(\psi_{h}\right)\right\|_{L^{\infty}\left(\mathbb{T}^{2}\right)} \\
& \leq C\left\|\nabla e_{h}^{i}\right\|\left\|\nabla e_{h}^{j}\right\| \sup _{K \in \mathcal{T}_{h}} \min _{\gamma=1,2,3}\left|\cos \alpha_{K, \gamma}\right|\left\|\psi_{h}\right\|_{L^{\infty}\left(\mathbb{T}^{2}\right)} .
\end{aligned}
$$

The inverse estimate (10) and Poincaré's inequality guarantee that

$$
\left\|\psi_{h}\right\|_{L^{\infty}\left(\mathbb{T}^{2}\right)} \leq C \log h_{\min }^{-1}\left\|\nabla \psi_{h}\right\|,
$$

and the definition of $\psi_{h}$ provides the estimate

$$
\left\|\nabla \psi_{h}\right\| \leq\left\|\vartheta_{h}^{j} \eta_{h}\right\| \leq C .
$$


The combination of the estimates and the definition of logarithmically right-angled triangulations proves the asserted limit for $\Lambda_{4}$. The same arguments lead to the assertion for $\Lambda_{5}$ (where the uniform bounds $\left\|e_{h}^{i}\right\|_{L^{\infty}\left(\mathbb{T}^{2}\right)} \leq 1$ may be employed for a more direct proof).

Lemma 4.7. Let $1 \leq i \leq k$ and $f_{h}^{i} \in \mathcal{S}_{\#}^{1}\left(\mathcal{T}_{h}\right)$ be as in Lemma 4.5. There exist $\left(t_{\iota}^{i}\right)_{\iota \in \mathbb{N}} \subset \mathbb{R}$ and $\left(y_{\iota}^{i}\right)_{\iota \in \mathbb{N}} \subset \mathbb{T}^{2}$ such that $\sum_{\iota \in \mathbb{N}}\left|t_{\iota}^{i}\right|^{2 / 3} \leq C$ and for an appropriate subsequence (which is not relabeled) and every $\eta \in C^{\infty}\left(\mathbb{T}^{2}\right)$ we have, as $h \rightarrow 0$,

$$
\left(f_{h}^{i} ; \mathcal{I}_{h} \eta\right)_{h} \rightarrow \sum_{\iota \in \mathbb{N}} t_{\iota}^{i} \eta\left(y_{\iota}^{i}\right)
$$

Proof. We define $F_{h} \in C\left(\mathbb{T}^{2}\right)^{*}$ by setting for all $\eta \in C\left(\mathbb{T}^{2}\right)$,

$$
F_{h}(\eta):=\left(f_{h}^{i} ; \mathcal{I}_{h} \eta\right)_{h} .
$$

Since $F_{h}$ is uniformly bounded in $C\left(\mathbb{T}^{2}\right)^{*}$ there exists $F \in C\left(\mathbb{T}^{2}\right)^{*}$ such that (for a subsequence) we have $F_{h} \rightarrow^{*} F$ in $C\left(\mathbb{T}^{2}\right)^{*}$. We fix $\delta>0$ and define

$$
\Upsilon_{\delta, h}:=\left\{z \in \mathcal{N}_{h}: h_{z} \max _{j=1,2, \ldots, k}\left\|\nabla e_{h}^{j}\right\|_{L^{\infty}\left(\omega_{z}\right)}>\delta\right\} .
$$

Then, using that $h_{z}\left\|\nabla e_{h}^{j}\right\|_{L^{\infty}\left(\omega_{z}\right)} \leq C\left\|\nabla e_{h}^{j}\right\|_{L^{2}\left(\omega_{z}\right)}$ for each $z \in \mathcal{N}_{h}$ we infer that

$$
\begin{aligned}
\operatorname{card} \Upsilon_{\delta, h} & \leq \delta^{-2} \sum_{z \in \mathcal{N}_{h}} h_{z}^{2} \max _{j=1,2, \ldots, k}\left\|\nabla e_{h}^{j}\right\|_{L^{\infty}\left(\omega_{z}\right)}^{2} \\
& \leq C \delta^{-2} \sum_{z \in \mathcal{N}_{h}} \sum_{j=1}^{k}\left\|\nabla e_{h}^{j}\right\|_{L^{2}\left(\omega_{z}\right)}^{2} \leq C \delta^{-2} \sum_{j=1}^{k}\left\|\nabla e_{h}^{j}\right\|^{2} \leq C \delta^{-2} ;
\end{aligned}
$$

i.e., the cardinality of the set $\Upsilon_{\delta, h}$ is uniformly bounded with respect to $h$ and therefore, for an appropriate subsequence which is not relabeled, we have

$$
\Upsilon_{\delta, h} \rightarrow \Upsilon_{\delta}=\left\{x_{1}^{\delta}, x_{2}^{\delta}, \ldots, x_{L^{\delta}}^{\delta}\right\}
$$

as $h \rightarrow 0$. With $F_{h}^{z}:=F_{h}\left(\varphi_{z}\right) \in \mathbb{R}$ for each $z \in \mathcal{N}_{h}$ we have

$$
F_{h}(\eta)=\sum_{z \in \mathcal{N}_{h}} F_{h}^{z} \eta(z)=\sum_{z \in \Upsilon_{\delta, h}} F_{h}^{z} \eta(z)+\sum_{z \in \mathcal{N}_{h} \backslash \Upsilon_{\delta, h}} F_{h}^{z} \eta(z)=: F_{\delta, h}^{b}(\eta)+F_{\delta, h}^{g}(\eta) .
$$

With the estimates of Lemma 4.5 we infer that

$$
\begin{aligned}
\left|F_{\delta, h}^{g}(\eta)\right| & \leq C h\|\nabla \eta\|_{L^{\infty}\left(\mathbb{T}^{2}\right)}+C \sum_{z \in \mathcal{N}_{h} \backslash \Upsilon_{\delta, h}} h_{z}\left\|\nabla e_{h}^{i}\right\|_{L^{\infty}\left(\omega_{z}\right)} \gamma_{h, z, i}^{2}|\eta(z)| \\
& \leq C h\|\nabla \eta\|_{L^{\infty}\left(\mathbb{T}^{2}\right)}+C \delta\|\eta\|_{L^{\infty}\left(\mathbb{T}^{2}\right)} .
\end{aligned}
$$

In particular we have (after passage to a subsequence) that $F_{\delta, h}^{g}-^{*} F_{\delta}^{g}$ in $C\left(\mathbb{T}^{2}\right)^{*}$ as $h \rightarrow 0$ with $F_{\delta}^{g} \in C\left(\mathbb{T}^{2}\right)^{*}$ such that $\left\|F_{\delta}^{g}\right\|_{C\left(\mathbb{T}^{2}\right)^{*}} \leq C \delta$. For $F_{\delta, h}^{b}$ we have that

$$
\left|F_{\delta, h}^{b}(\eta)\right| \leq C h\|\nabla \eta\|_{L^{\infty}\left(\mathbb{T}^{2}\right)}+C \sum_{z \in \Upsilon_{\delta, h}} \gamma_{h, z, i}^{3}|\eta(z)| .
$$

An application of Lemma B.2 shows that for a subsequence we have $F_{\delta, h}^{b} \rightarrow^{*} F_{\delta}^{b}=$ $\sum_{\iota=1}^{L^{\delta}} \rho_{\iota}^{\delta} \delta_{x_{\iota}^{\delta}}$ as $h \rightarrow 0$ for $\rho_{\iota}^{\delta} \in \mathbb{R}$ such that $\sum_{\iota=1}^{L^{\delta}}\left|\rho_{\iota}^{\delta}\right|^{2 / 3} \leq C$ independently of $\delta$. We thus have

$$
\left\|F-F_{\delta}^{b}\right\|_{C\left(\mathbb{T}^{2}\right)^{*}} \leq C \delta .
$$


Employing Lemma B.1 we verify the assertion.

Lemma 4.8. Suppose that $\left(u_{h}\right)_{h>0}$ is a bounded sequence in $W^{1,2}\left(\mathbb{T}^{2} ; \mathbb{R}^{n}\right)$ such that $u_{h} \in \mathcal{S}_{\#}^{1}\left(\mathcal{T}_{h}\right)^{n}$ for all $h>0$ and $u_{h}(z) \in N$ for all $z \in \mathcal{N}_{h}$. For each $h>0$ let $\left(e_{h}^{i}\right)_{i=1,2, \ldots, k}$ be an orthonormal frame for $u_{h}^{-1} T N$ which is optimal in the sense of Lemma 4.3 so that $\max _{i=1,2, \ldots, k}\left\|\nabla e_{h}^{i}\right\| \leq C$. Then, for every accumulation point $u \in W^{1,2}\left(\mathbb{T}^{2} ; \mathbb{R}^{n}\right)$ of the sequence $\left(u_{h}\right)_{h>0}$ and an appropriate subsequence, which is not relabeled in the following, we have:

(i) $u(x) \in N$ for almost every $x \in \mathbb{T}^{2}$ and

$$
u_{h} \rightarrow u \quad \text { in } W^{1,2}\left(\mathbb{T}^{2} ; \mathbb{R}^{n}\right) ;
$$

(ii) there exist $\left(e^{i}\right)_{i=1,2, \ldots, k} \subset W^{1,2}\left(\mathbb{T}^{2} ; \mathbb{R}^{n}\right)$ such that

$$
e_{h}^{i} \rightarrow e^{i} \quad \text { in } W^{1,2}\left(\mathbb{T}^{2} ; \mathbb{R}^{n}\right)
$$

and $\left(e^{i}\right)_{i=1,2, \ldots, k}$ is an orthonormal frame for $u^{-1} T N$, i.e., for almost every $x \in \mathbb{T}^{2}$ the vectors $e^{1}(x), e^{2}(x), \ldots, e^{k}(x)$ form an orthonormal basis for $T_{u(x)} N$;

(iii) for $\omega^{i j}:=e^{j, T} \nabla e^{i} \in L^{2}\left(\mathbb{T}^{2} ; \mathbb{R}^{2}\right)$ we have

$$
\omega_{h}^{i j}, \bar{\omega}_{h}^{i j} \rightarrow \omega^{i j} \quad \text { in } L^{2}\left(\mathbb{T}^{2} ; \mathbb{R}^{n}\right) ;
$$

(iv) for $\vartheta^{i}:=e^{i, \mathrm{~T}} \nabla u \in L^{2}\left(\mathbb{T}^{2} ; \mathbb{R}^{2}\right)$ we have

$$
\vartheta_{h}^{i}, \bar{\vartheta}_{h}^{i} \rightarrow \vartheta^{i} \quad \text { in } L^{2}\left(\mathbb{T}^{2} ; \mathbb{R}^{2}\right) ;
$$

(v) there exist $b^{i j} \in W^{1,2}\left(\mathbb{T}^{2}\right)$ and $H^{i j} \in L^{2}\left(\mathbb{T}^{2} ; \mathbb{R}^{2}\right)$ such that for $a_{h}^{i j}, \widehat{b}_{h}^{i j}$, and $H_{h}^{i j}$ as in Lemma 4.4 we have

$$
a_{h}^{i j} \rightarrow 0 \text { in } W^{1,2}\left(\mathbb{T}^{2}\right), \quad \widehat{b}_{h}^{i j} \rightarrow b^{i j} \text { in } W^{1,2}\left(\mathbb{T}^{2}\right), \quad H_{h}^{i j} \rightarrow H^{i j} \text { in } L^{2}\left(\mathbb{T}^{2} ; \mathbb{R}^{2}\right)
$$

and $\omega^{i j}=\operatorname{Curl} b^{i j}+H^{i j}$.

Proof. (i) For every accumulation point $u \in W^{1,2}\left(\mathbb{T}^{2} ; \mathbb{R}^{n}\right)$ of the bounded sequence $\left(u_{h}\right)_{h>0}$ there exists a subsequence which we do not relabel such that $u_{h} \rightarrow u$ in $W^{1,2}\left(\mathbb{T}^{2} ; \mathbb{R}^{n}\right)$. Lemma 2.4 then implies that $u(x) \in N$ for almost every $x \in \mathbb{T}^{2}$.

(ii) Since each sequence $\left(e_{h}^{i}\right)_{h>0}$ is bounded in $W^{1,2}\left(\mathbb{T}^{2} ; \mathbb{R}^{n}\right)$, each of them admits a weak limit $e^{i} \in W^{1,2}\left(\mathbb{T}^{2} ; \mathbb{R}^{n}\right)$ of an appropriate subsequence. By successive extraction of subsequences we may assume that the same subsequence converges weakly for each $1 \leq i \leq k$. For $1 \leq i, j \leq k$ with $i \neq j$ we have $e_{h}^{i}(z) \cdot e_{h}^{j}(z)=0$ for all $z \in \mathcal{N}_{h}$ and the discrete Poincaré inequality (12) proves that

$$
\left\|e_{h}^{i} \cdot e_{h}^{j}\right\| \leq C h\left\|\nabla\left(e_{h}^{i} \cdot e_{h}^{j}\right)\right\| \leq C h\left\|e_{h}^{j, \mathrm{~T}} \nabla e_{h}^{i}+e_{h}^{i, \mathrm{~T}} \nabla e_{h}^{j}\right\| \leq C h,
$$

where we have used that $\left|e_{h}^{i}\right|,\left|e_{h}^{j}\right| \leq 1$ almost everywhere in $\mathbb{T}^{2}$ and $\left\|\nabla e_{h}^{i}\right\|,\left\|\nabla e_{h}^{j}\right\| \leq$ $C$, independently of $h$. Hence, $e_{h}^{i} \cdot e_{h}^{j} \rightarrow 0$ in $L^{2}\left(\mathbb{T}^{2}\right)$ and in particular, $e_{h}^{i}(x) \cdot e_{h}^{j}(x) \rightarrow$ 0 for almost every $x \in \mathbb{T}^{2}$. Since also $e_{h}^{i}(x) \rightarrow e^{i}(x)$ and $e_{h}^{j}(x) \rightarrow e^{j}(x)$ for almost every $x \in \mathbb{T}^{2}$ we deduce that $e^{i} \cdot e^{j}=0$ almost everywhere in $\mathbb{T}^{2}$. Similarly, using that $\left|e_{h}^{i}(z)\right|=1$ for all $z \in \mathcal{N}_{h}$ we estimate

$$
\left\|\left|e_{h}^{i}\right|^{2}-1\right\| \leq C h\left\|\nabla\left|e_{h}^{i}\right|^{2}\right\| \leq C h\left\|e_{h}^{i, \mathrm{~T}} \nabla e_{h}^{i}\right\| \leq C h,
$$

which implies $\left|e_{h}^{i}\right|^{2} \rightarrow 1$ in $L^{2}\left(\mathbb{T}^{2}\right)$ and hence $\left|e^{i}\right|=1$ almost everywhere in $\mathbb{T}^{2}$. It remains to show that for $\ell=k+1, \ldots, n$ we have $e^{i} \cdot\left(\nu^{\ell} \circ u\right)=0$ almost everywhere in $\mathbb{T}^{2}$. Since $\nu^{\ell}$ is locally $C^{1}$ and $e_{h}^{i}(x) \rightarrow e^{i}(x), u_{h}(x) \rightarrow u(x)$ as $h \rightarrow 0$ for almost 
every $x \in \mathbb{T}^{2}$ it suffices to show that for every $\delta>0$ and almost every $x \in M$ there exists $h_{0}=h_{0}(x)$ such that for all $h<h_{0}$ we have

$$
\left|e_{h}^{i}(x) \cdot \nu^{\ell}\left(u_{h}(x)\right)\right| \leq \delta .
$$

Fix $\delta>0$ and define for $h>0$,

$$
\Upsilon_{\delta, h}:=\left\{z \in \mathcal{N}_{h}:\left\|\nabla u_{h}\right\|_{L^{2}\left(\omega_{z}\right)}+\left\|\nabla e_{h}^{i}\right\|_{L^{2}\left(\omega_{z}\right)}>\delta\right\} .
$$

Then, card $\Upsilon_{\delta, h} \leq C \delta^{-2}$ for all $h>0$ and hence $\Upsilon_{\delta, h} \rightarrow \Upsilon_{\delta}=\left\{x_{1}^{\delta}, x_{2}^{\delta}, \ldots, x_{L_{\delta}}^{\delta}\right\}$ for $x_{1}^{\delta}, x_{2}^{\delta}, \ldots, x_{L_{\delta}}^{\delta} \in \mathbb{T}^{2}$ as $h \rightarrow 0$. For each $x \in \mathbb{T}^{2} \backslash \Upsilon_{\delta}$ there exists $h_{0}$ such that for every $h<h_{0}$ there exists $z_{h}^{x} \in \mathcal{N}_{h} \backslash \Upsilon_{\delta, h}$ such that $x \in \omega_{z_{h}^{x}}$. Then we have, using that $e_{h}^{i}(z) \cdot \nu^{\ell}\left(u_{h}(z)\right)=0$ for all $z \in \mathcal{N}_{h}$, that

$$
\begin{aligned}
\left|e_{h}^{i}(x) \cdot \nu^{\ell}\left(u_{h}(x)\right)\right|= & \mid e_{h}^{i}\left(z_{h}^{x}\right) \cdot\left[\nu^{\ell}\left(u_{h}(x)\right)-\nu^{\ell}\left(u_{h}\left(z_{h}^{x}\right)\right)\right] \\
& \quad+\left[e_{h}^{i}\left(z_{h}^{x}\right)-e_{h}^{i}(x)\right] \cdot \nu^{\ell}\left(u_{h}(x)\right) \mid \\
\leq & \left\|D \bar{\nu}^{\ell}\right\|_{L^{\infty}\left(B_{h_{0}}(u(x))\right)}\left|u_{h}(x)-u_{h}\left(z_{h}^{x}\right)\right|+\left|e_{h}^{i}\left(z_{h}^{x}\right)-e_{h}^{i}(x)\right| \\
\leq & C h\left(\left\|\nabla u_{h}\right\|_{L^{\infty}\left(\omega_{z_{h}^{x}}\right)}+\left\|\nabla e_{h}^{i}\right\|_{L^{\infty}\left(\omega_{\left.z_{h}^{x}\right)}\right)}\right) \\
\leq & C\left(\left\|\nabla u_{h}\right\|_{L^{2}\left(\omega_{z_{h}^{x}}\right)}+\left\|\nabla e_{h}^{i}\right\|_{L^{2}\left(\omega_{z_{h}^{x}}\right)}\right) \\
\leq & C \delta,
\end{aligned}
$$

which proves the statement.

(iii) For all $\eta \in C^{\infty}\left(\mathbb{T}^{2} ; \mathbb{R}^{2}\right)$ we have, using $e_{h}^{i} \rightarrow e^{i}$ in $L^{2}\left(\mathbb{T}^{2} ; \mathbb{R}^{n}\right)$ and $\nabla e_{h}^{j, \alpha} \rightarrow$ $\nabla e^{j, \alpha}$ in $L^{2}\left(\mathbb{T}^{2} ; \mathbb{R}^{2}\right), \alpha=1,2, \ldots, n$, that

$$
\begin{aligned}
\left(\bar{\omega}_{h}^{i j}-\omega^{i j} ; \eta\right) & =\sum_{\alpha=1}^{n}\left(e_{h}^{j, \alpha} \nabla e_{h}^{i, \alpha}-e^{j, \alpha} \nabla e^{i, \alpha} ; \eta\right) \\
& =\sum_{\alpha=1}^{n}\left\{\left(\left[e_{h}^{j, \alpha}-e^{j, \alpha}\right] \nabla e_{h}^{i, \alpha} ; \eta\right)+\left(\left[\nabla e_{h}^{j, \alpha}-\nabla e^{j, \alpha}\right] \cdot e^{i, \alpha} ; \eta\right)\right\} \rightarrow 0
\end{aligned}
$$

as $h \rightarrow 0$. Therefore $\bar{\omega}_{h}^{i j} \rightarrow \omega^{i j}$ in $L^{2}\left(\mathbb{T}^{2} ; \mathbb{R}^{2}\right)$ as $h \rightarrow 0$. Using

$$
\left\|\mathbf{A}\left(e_{h}^{j, \alpha}\right)-e_{h}^{j, \alpha} \mathbf{I}\right\| \leq C h\left\|\nabla e_{h}^{j, \alpha}\right\|
$$

we find that $\mathbf{A}\left(e_{h}^{j, \alpha}\right) \rightarrow e^{j, \alpha} \mathbf{I}$ in $L^{2}\left(\mathbb{T}^{2} ; \mathbb{R}^{2 \times 2}\right)$ for $\alpha=1,2, \ldots, n$ and thus also $\omega_{h}^{i j} \rightarrow \omega^{i j}$ in $L^{2}\left(\mathbb{T}^{2} ; \mathbb{R}^{2}\right)$.

(iv) This follows exactly as the assertion in (iii).

(v) For $\phi \in C^{\infty}\left(\mathbb{T}^{2}\right)$ and $\phi_{h}:=\mathcal{I}_{h} \phi \in \mathcal{S}_{\#}^{1}\left(\mathcal{T}_{h}\right)$ we have

$$
\left(\nabla a_{h}^{i j} ; \nabla \phi\right)=\left(\nabla a_{h}^{i j} ; \nabla \phi_{h}\right)+\left(\nabla a_{h}^{i j} ; \nabla\left[\phi-\phi_{h}\right]\right)
$$

and the second term on the right-hand side vanishes as $h \rightarrow 0$ owing to uniform boundedness of $a_{h}^{i j}$ in $W^{1,2}\left(\mathbb{T}^{2}\right)$ and nodal interpolation results. By definition of $a_{h}^{i j}$ and Lemma 4.3, we have

$$
\left(\nabla a_{h}^{i j} ; \nabla \phi_{h}\right)=\left(\omega_{h}^{i j} ; \nabla \phi_{h}\right)=\Lambda_{4}\left(e_{h}^{i}, e_{h}^{j}, \phi_{h}\right)+\Lambda_{5}\left(e_{h}^{i}, e_{h}^{j}, \phi_{h}\right) .
$$

The definition of $\Lambda_{4}$ and $\Lambda_{5}$ and the estimates for the operator $\mathbf{A}$ in Subsection 2.3 imply

$$
\left|\Lambda_{4}\left(e_{h}^{i}, e_{h}^{j}, \phi_{h}\right)+\Lambda_{5}\left(e_{h}^{i}, e_{h}^{j}, \phi_{h}\right)\right| \leq C h\left\|\nabla e_{h}^{i}\right\|\left\|\nabla e_{h}^{j}\right\|\left\|\nabla \phi_{h}\right\|_{L^{\infty}(M)}
$$


and thus $a_{h}^{i j} \rightarrow 0$ as $h \rightarrow 0$. Since $H_{h}^{i j}$ is a bounded sequence in a finite-dimensional space there exists $H^{i j}$ such that, for an appropriate subsequence, we have $H_{h}^{i j} \rightarrow$ $H^{i j}$ in $L^{2}\left(\mathbb{T}^{2} ; \mathbb{R}^{2}\right)$. Since $\left\|\operatorname{Curl}_{\mathcal{T}_{h}} b_{h}^{i j}\right\|$ is bounded uniformly we find, using (16), that $b_{h}^{i j}-\widehat{b}_{h}^{i j} \rightarrow 0$ in $L^{2}\left(\mathbb{T}^{2}\right)$ and $\widehat{b}_{h}^{i j} \rightarrow b^{i j}$ for some $b^{i j} \in W^{1,2}\left(\mathbb{T}^{2}\right)$ (and an appropriate subsequence). For every $\psi \in C^{\infty}\left(\mathbb{T}^{2} ; \mathbb{R}^{2}\right)$ and $\psi_{h}:=\mathcal{I}_{h} \psi$ we find with an elementwise integration by parts as in the proof of Lemma 4.5 that

$$
\begin{aligned}
\left(\omega_{h}^{i j} ; \psi_{h}\right)= & \left(\operatorname{Curl}_{\mathcal{T}_{h}} b_{h}^{i j} ; \psi_{h}\right)+\left(H_{h}^{i j} ; \psi_{h}\right)+\left(\nabla a_{h}^{i j} ; \psi_{h}\right) \\
= & -\left(b_{h}^{i j} ; \operatorname{curl} \psi_{h}\right)+\left(H_{h}^{i j} ; \psi_{h}\right) \\
& +\sum_{E \in \mathcal{E}_{h}} \int_{E}\left[b_{h}^{i j}\right]\left(\psi_{h}-c_{E}\right) \tau_{E} \mathrm{~d} t+\left(\nabla a_{h}^{i j} ; \psi_{h}\right),
\end{aligned}
$$

where $c_{E} \in \mathbb{R}^{2}$ is an arbitrary constant vector for each $E \in \mathcal{E}_{h}$. Arguing as in (32), passing to the limit for $h \rightarrow 0$, and integrating by parts, we verify that $\omega^{i j}=$ Curl $b^{i j}+H^{i j}$. This finishes the proof of the lemma.

The following result is based on P. L. Lions' concentrated compactness principle [32. For a discussion of the assertion in the considered periodic setting we refer to 23 .

Lemma 4.9 (23, Equation (2.4)]). Suppose that $\left(b_{h}\right)_{h>0},\left(e_{h}\right)_{h>0}$ and $\left(f_{h}\right)_{h>0}$ are bounded sequences in $W^{1,2}\left(\mathbb{T}^{2}\right)$ with weak limits $b, e, f \in W^{1,2}\left(\mathbb{T}^{2}\right)$, respectively, and assume that $e_{h}$ is bounded in $L^{\infty}\left(\mathbb{T}^{2}\right)$. Then, there exist $\left(s_{\iota}\right)_{\iota \in \mathbb{N}} \subset \mathbb{R}$ satisfying $\sum_{\iota \in \mathbb{N}}\left|s_{\iota}\right| \leq C$ and $\left(x_{\iota}\right)_{\iota \in \mathbb{N}} \subset \mathbb{T}^{2}$ such that for (a subsequence and) all $\eta \in C^{\infty}\left(\mathbb{T}^{2}\right)$, we have, as $h \rightarrow 0$,

$$
\left(\operatorname{Curl} b_{h} ; e_{h} \eta \nabla f_{h}\right) \rightarrow(\operatorname{Curl} b ; e \eta \nabla f)+\sum_{\iota \in \mathbb{N}} s_{\iota} \eta\left(x_{\iota}\right) .
$$

4.5. Statement of the main result. We are now in a position to prove the asserted weak convergence result for a sequence of periodic, almost discrete harmonic maps.

Theorem 4.10. Let $\left(\mathcal{T}_{h}\right)_{h>0}$ be a sequence of regular, logarithmically right-angled triangulations of $\mathbb{T}^{2}$ and let $\left(u_{h}\right)_{h>0}$ be such that for all $h>0$ we have $u_{h} \in$ $\mathcal{S}_{\#}^{1}\left(\mathcal{T}_{h}\right)^{n}, u_{h}(z) \in N$ for all $z \in \mathcal{N}_{h}$, and

$$
\left\|\nabla u_{h}\right\| \leq C
$$

Suppose that there exists a sequence $\left(\varepsilon_{h}\right)_{h>0}$ such that $\varepsilon_{h} \rightarrow 0$ as $h \rightarrow 0$ and for all $v_{h} \in \mathcal{S}_{\#}^{1}\left(\mathcal{T}_{h}\right)^{n}$ satisfying $v_{h}(z) \in T_{u_{h}(z)} N$ for all $z \in \mathcal{N}_{h}$, we have

$$
\left|\left(\nabla u_{h} ; \nabla v_{h}\right)\right| \leq \varepsilon_{h}\left\|\nabla v_{h}\right\| .
$$

Then every weak accumulation point of $\left(u_{h}\right)_{h>0} \subset W^{1,2}\left(\mathbb{T}^{2} ; \mathbb{R}^{n}\right)$ is a harmonic map into $N$.

Proof. We let $u \in W^{1,2}\left(\mathbb{T}^{2} ; \mathbb{R}^{n}\right)$ be a weak accumulation point of $\left(u_{h}\right)_{h>0}$, and we do not relabel the corresponding subsequence so that $u_{h} \rightarrow u$ as $h \rightarrow 0$. Let $\eta \in C^{\infty}\left(\mathbb{T}^{2}\right)$ and for $h>0$ set $\eta_{h}:=\mathcal{I}_{h} \eta$. For each $h>0$ let $\left(e_{h}^{i}\right)_{i=1,2, \ldots, k}$ be an orthonormal frame for $u_{h}^{-1} T N$ which is optimal in the sense of Lemma 4.3. Then, 
Lemma 4.4 and Lemma 4.5 imply that

$$
\begin{aligned}
& \left(\nabla u_{h} ; \nabla \mathcal{I}_{h}\left[\eta_{h} e_{h}^{i}\right]\right) \\
& =\sum_{j=1}^{k}\left\{\left(\operatorname{Curl} \widehat{b}_{h}^{i j} \cdot \bar{\vartheta}_{h}^{j} ; \eta_{h}\right)+\left(H_{h}^{i j} \cdot \bar{\vartheta}_{h}^{j} ; \eta_{h}\right)\right\}+\left(\vartheta_{h}^{i} ; \nabla \eta_{h}\right) \\
& \quad+\sum_{j=1}^{k} \Lambda_{4,5}\left(e_{h}^{i}, e_{h}^{j}, \psi_{h}^{j}\right)+\left(f_{h}^{i} ; \eta_{h}\right)_{h} .
\end{aligned}
$$

With the limits $b^{i j}, H^{i j}, \vartheta^{j}$ and $\omega^{i j}$ of appropriate subsequences identified in Lemmas 4.7 4.8 and 4.9 and since $\varepsilon_{h} \rightarrow 0$, we find that

$$
0=\sum_{j=1}^{k}\left\{\left(\operatorname{Curl} b^{i j} \cdot \vartheta^{j} ; \eta\right)+\left(H^{i j} \cdot \vartheta^{j} ; \eta\right)\right\}+\left(\vartheta^{i} ; \nabla \eta\right)+\sum_{\iota \in \mathbb{N}} s_{\iota} \eta\left(x_{\iota}\right)+\sum_{\iota \in \mathbb{N}} t_{\iota} \eta\left(y_{\iota}\right)
$$

and $\omega^{i j}=\operatorname{Curl} b^{i j}+H^{i j}$ so that

$$
\sum_{j=1}^{k}\left(\omega^{i j} \cdot \vartheta_{h}^{j} ; \eta\right)+\left(\vartheta^{i} ; \nabla \eta\right)=\sum_{\iota \in \mathbb{N}} s_{\iota} \eta\left(x_{\iota}\right)+\sum_{\iota \in \mathbb{N}} t_{\iota} \eta\left(y_{\iota}\right) .
$$

The left-hand side of this identity belongs to $L^{1}(M)+H^{-1}(M)$, which does not contain Dirac measures; see 23] for details. Therefore, $s_{\iota}=t_{\iota}=0$ for all $\iota \in \mathbb{N}$ and Proposition 2.1 implies that the weak limit $u$ is a harmonic map into $N$.

\section{Reduction of the general Case to a PERIodic SEtting}

In this section we discuss the generalization of Theorem 4.10 to general domains. The key ingredient in the proof is a discrete version of a periodification argument from [23].

Theorem 5.1. Suppose that $M=M \subset \mathbb{R}^{2} \times\{0\}$ is a bounded Lipschitz domain in $\mathbb{R}^{2}$ with polyhedral boundary and assume that $\left(\mathcal{T}_{h}\right)_{h>0}$ is a sequence of logarithmically right-angled, regular triangulations of $M$. Let $\left(u_{h}\right)_{h>0}$ be such that for each $h>0$ we have $u_{h} \in \mathcal{S}^{1}\left(\mathcal{T}_{h}\right)^{n}, u_{h}(z) \in N$ for all $z \in \mathcal{N}_{h},\left.u_{h}\right|_{\Gamma_{\mathrm{D}, h}}=u_{\mathrm{D}, h}$, and

$$
\left\|\nabla u_{h}\right\| \leq C \text {. }
$$

Let $\left(\varepsilon_{h}\right)_{h>0}$ be such that $\varepsilon_{h} \rightarrow 0$ as $h \rightarrow 0$ and for all $v_{h} \in \mathcal{S}_{\mathrm{D}}^{1}\left(\mathcal{T}_{h}\right)^{n}$ satisfying $v_{h}(z) \in T_{u_{h}(z)} N$ for all $z \in \mathcal{N}_{h}$ we have

$$
\left|\left(\nabla u_{h} ; \nabla v_{h}\right)\right| \leq \varepsilon_{h}\left\|\nabla v_{h}\right\| .
$$

Then every weak accumulation point of $\left(u_{h}\right)_{h>0} \subset W^{1,2}\left(M ; \mathbb{R}^{n}\right)$ is a harmonic map into $N$ with $\left.u\right|_{\Gamma_{\mathrm{D}}}=u_{\mathrm{D}}$.

Proof. Let $u \in W^{1,2}\left(M ; \mathbb{R}^{n}\right)$ be a weak accumulation point of the sequence $\left(u_{h}\right)_{h>0}$. Then, Lemma 2.4 implies that $u(x) \in N$ for almost every $x \in M$. Moreover, weak continuity of the trace operator yields $\left.u\right|_{\Gamma_{\mathrm{D}}}=u_{\mathrm{D}}$. It remains to show that $u$ is a harmonic map, i.e., that

$$
(\nabla u ; \nabla v)=0
$$

for all $v \in W_{0}^{1,2}\left(M ; \mathbb{R}^{n}\right)$ satisfying $v(x) \in T_{u(x)} N$ for almost every $x \in M$. Given some fixed $\delta>0$ it suffices to prove this identity for all $v \in W_{0}^{1,2}\left(Q ; R^{n}\right)$ for all cubes $Q \subset M$ with sides of length at most $\delta$ and parallel to the coordinate axes. 
We fix such a cube $Q$ and may assume without loss of generality that $Q=Q_{1 / 4}(a)$ is centered at $a=(1 / 4,1 / 4)$ and has sides of length $1 / 4$. Also, we may assume that $Q_{1 / 2}(a) \subset M$. For $h$ sufficiently small we consider the cube $Q_{1 / 2-2 h}(a)$ and introduce the subset $\widetilde{\mathcal{T}}_{h}$ of the triangulation $\mathcal{T}_{h}$ by setting

$$
\widetilde{\mathcal{T}}_{h}:=\left\{K \in \mathcal{T}_{h}: K \cap Q_{1 / 2-2 h}(a) \neq \emptyset\right\} .
$$

Then, $\widetilde{\mathcal{T}}_{h}$ covers $Q_{1 / 2-2 h}(a)$ and is contained in $Q_{1 / 2}(a)$; cf. the left plot of Figure 3 .

To reduce the current situation to the periodic setting discussed in the previous section, we (1) extend $\widetilde{\mathcal{T}}_{h}$ to a regular triangulation $\widehat{\mathcal{T}}_{h}$ of $(0,1 / 2)^{2},(2)$ extend $\left.u_{h}\right|_{\cup \widetilde{\mathcal{T}}_{h}}$ to a function $\widehat{u}_{h} \in \mathcal{S}^{1}\left(\widehat{\mathcal{T}}_{h}\right)^{n}$ such that $\widehat{u}_{h}(z) \in N$ for all $z \in \widehat{\mathcal{N}}_{h}$, the nodes of the triangulation $\widehat{\mathcal{T}}_{h}$, and such that $\left\|\nabla \widehat{u}_{h}\right\|_{L^{2}\left((0,1 / 2)^{2}\right)} \leq C$, and (3) reflect $\widehat{u}_{h}$ across the lines $\{1 / 2\} \times \mathbb{R}$ and $\mathbb{R} \times\{1 / 2\}$ in order to obtain a periodic function on $[0,1]^{2}$ to which we can apply the theory of the previous section. Since the test functions will only be supported in $Q$, where the extended and the original triangulation coincide, it is not necessary to guarantee that the extended triangulation is logarithmically right-angled (outside of $Q$ ).
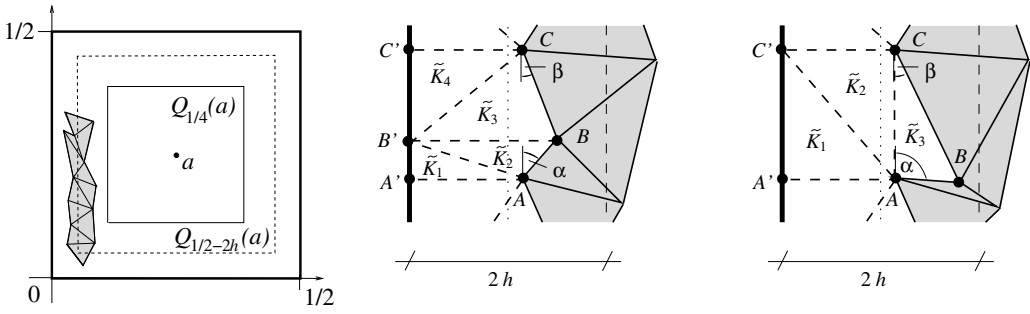

Figure 3. Cubes $Q_{1 / 4}(a)$ and $Q_{1 / 2-2 h}(a)$ for $a=(1 / 4,1 / 4)$ and typical triangles of the subtriangulation $\widetilde{\mathcal{T}}_{h}$ (left). Typical scenarios in the extension of the triangulation $\widetilde{\mathcal{T}}_{h}$ (shaded) to a triangulation of $(0,1 / 2)^{2}$ (middle and right). The angle $\alpha$ is not critical in the left plot and is critical in the right one.

Step 1. The task to extend $\widetilde{\mathcal{T}}_{h}$ to a regular triangulation of $(0,1 / 2)^{2}$ is simple if $\mathcal{T}_{h}$ is a uniform triangulation consisting of halved squares with sides parallel to the coordinate axes. The general case is slightly more involved and, in order to guarantee shape-regularity of the extended triangulation, we discuss a few typical scenarios. Consider first the situation depicted in the middle plot of Figure 3 and assume that the angles $\alpha, \beta$ are not critical in the sense that they satisfy $-\pi / 2+c_{0} \leq \alpha, \beta \leq \pi / 2-c_{0}$ with a uniform (small) constant $c_{0}>0$. We then introduce the new triangles $\widetilde{K}_{1}, \widetilde{K}_{2}, \widetilde{K}_{3}, \widetilde{K}_{4}$ as shown. A typical critical angle $\alpha$ is depicted in the right plot of Figure 3 . In this case, we connect the points $A$ and $C$ to obtain a new triangle $\widetilde{K}_{3}$. We can then proceed as in the previous case. Next, we examine a typical situation at a corner of the cube $Q_{1 / 2-2 h}(a)$. Again, the construction of the extension depends on the angle $\alpha$ defined in Figure 4. In the left plot, $\alpha$ satisfies $-\pi / 2+c_{0} \leq \alpha \leq \pi / 2-c_{0}$ and we introduce the new triangles $\widetilde{K}_{1}, \widetilde{K}_{2}, \widetilde{K}_{3}, \widetilde{K}_{4}$. The case of a critical angle is depicted in the right plot of Figure 4 We connect the vertices $A$ and $B$ to introduce the new triangle $\widetilde{K}_{5}$. We are then in 

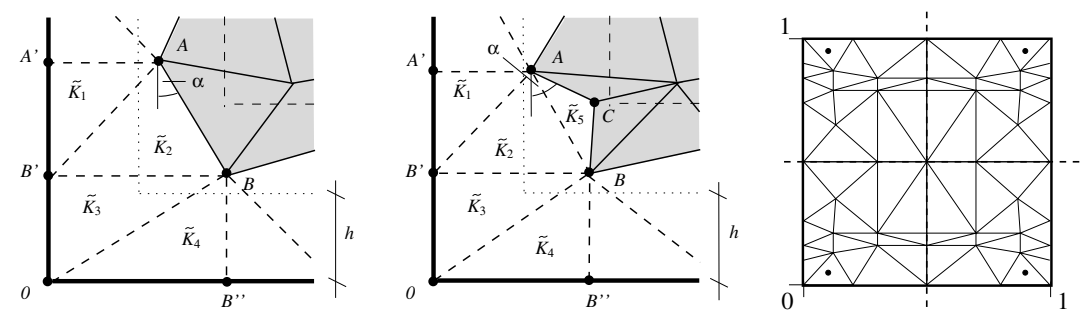

Figure 4. Typical scenarios in the extension of the triangulation $\widetilde{\mathcal{T}}_{h}$ (shaded) to a triangulation of $(0,1 / 2)^{2}$ at a corner. The angle $\alpha$ is not critical in the left plot and is critical in the right one (left and middle). Reflection of the triangulation $\widetilde{\mathcal{T}}_{h}$ of $(0,1 / 2)^{2}$ to obtain a ("periodic") triangulation of $\mathbb{T}^{2}$ with fundamental domain $[0,1]^{2}$ (right; the dot is included for better visualization).

the situation described above. We remark that in case that there are triangles with $|A-B| \ll h$, then the new triangles $\widetilde{K}_{i}$ can be refined further in order to maintain shape regularity.

Step 2. In the situations discussed above, we extend $u_{h}$ by setting $\widehat{u}_{h}\left(A^{\prime}\right):=u_{h}(A)$, $\widehat{u}_{h}\left(B^{\prime}\right):=u_{h}(B), \widehat{u}_{h}\left(C^{\prime}\right):=u_{h}(C)$ and $\widehat{u}_{h}\left(A^{\prime}\right):=u_{h}(A), \widehat{u}_{h}\left(C^{\prime}\right):=u_{h}(C)$ in the situations depicted in the left and right plots of Figure 3, respectively. We set $\widehat{u}_{h}\left(A^{\prime}\right):=u_{h}(A), \widehat{u}_{h}\left(B^{\prime}\right)=\widehat{u}_{h}\left(B^{\prime \prime}\right)=\widehat{u}_{h}(0):=u_{h}(B), \widehat{u}_{h}\left(C^{\prime}\right):=u_{h}(C)$ in the scenarios shown in Figure 4 . In order to show that we do not increase the gradient of $u_{h}$ we notice that, e.g., in the situation of the right plot of Figure 3 we have

$$
\begin{aligned}
\left|\nabla \widehat{u}_{h}\right|_{\widetilde{K}_{2}}\left|\leq h^{-1}\right| \widehat{u}_{h}\left(C^{\prime}\right)-\widehat{u}_{h}(A) \mid & \leq h^{-1}\left(\left|u_{h}(A)-u_{h}(B)\right|+\left|u_{h}(B)-u_{h}(C)\right|\right) \\
& \leq\left.\left|\nabla u_{h}\right|_{K_{A}}|+| \nabla u_{h}\right|_{K_{B}} \mid,
\end{aligned}
$$

where $K_{A}, K_{B} \in \widetilde{\mathcal{T}}_{h}$ are such that $A \in K_{A}$ and $B \in K_{B}$.

Step 3 . We reflect the triangulation $\widehat{\mathcal{T}}_{h}$ and the function $\widehat{u}_{h}$ across the lines $\{1 / 2\} \times \mathbb{R}$ and $\mathbb{R} \times\{1 / 2\}$ to obtain a triangulation $\mathcal{T}_{h}^{\#}$ of $\mathbb{T}^{2}$ and a function $u_{h}^{\#} \in \mathcal{S}_{\#}^{1}\left(\mathcal{T}_{h}^{\#}\right)$ periodic function on $[0,1]^{2}$; cf. Figure 4

We may now apply Theorem 4.10 to the sequence $\left(u_{h}^{\#}\right)$. The only necessary modification is that test functions are supported in $Q_{1 / 4}(a)$. This shows that $u$ is harmonic in $Q_{1 / 4}(a)$ and finishes the proof of the theorem.

\section{NumERICAL EXPERIMENT}

Given a polyhedral approximation $M_{h}$ of a hypersurface $M \subset \mathbb{R}^{m+1}$ and employing the piecewise defined tangential gradient $\nabla_{M_{h}} \phi:=\nabla \phi^{e}-\left(\mu_{h} \cdot \nabla \phi^{e}\right) \mu_{h}$, where $\mu_{h}$ denotes a piecewise constant unit normal to $M_{h}, \phi^{e}$ an extension of $\phi$ to an open neighbourhood of $M_{h}$, and $\nabla$ the gradient operator in $\mathbb{R}^{m+1}$, obvious modifications of Algorithm A lead to an approximation scheme for harmonic maps between curved surfaces $M$ and $N$. Convergence of the resulting approximation can be studied by employing the lifting operator and corresponding estimates of [19]; we refer the reader to 8 for a detailed discussion.

We choose $M=N=T_{1,1 / 4}$ to be a two-dimensional torus with radii $r_{1}=1$ and $r_{2}=1 / 4$. For a triangulation containing 1024 triangles of diameter $h \approx 0.006$ that 

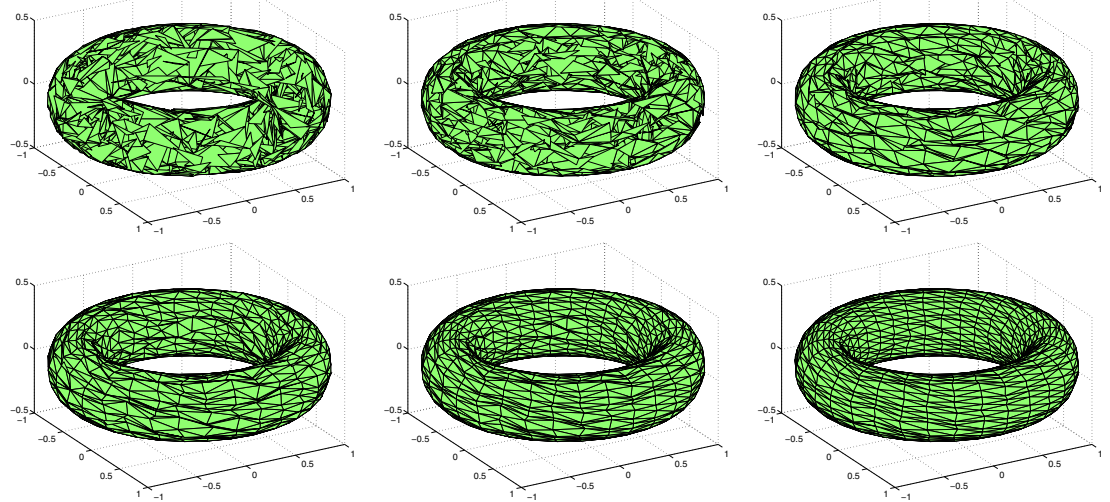

Figure 5. Deformations of the torus $T_{1,1 / 4}$ defined through mapped triangulations $\mathcal{T}_{h}^{i}$ of $\mathcal{T}_{h}$ under $u_{h}^{i}$ after $0,30,60,90,120$, and 150 iterations of Algorithm A (from left to right and top to bottom).

defines the approximation $M_{h}$ of $M$ we define the initial vector field $u_{h}^{0} \in \mathcal{S}^{1}\left(\mathcal{T}_{h}\right)^{3}$ satisfying $u_{h}^{0}(z) \in T_{1,1 / 4}$ for all $z \in \mathcal{N}_{h}$ by perturbing the identity on $T_{1,1 / 4}$; i.e., we set

$$
u_{h}^{0}(z):=\pi_{T_{1,1 / 4}}\left(z+\xi_{h}(z) / 3\right)
$$

for all $z \in \mathcal{N}_{h} \subseteq T_{1,1 / 4}$ and random vectors $\xi_{h}(z)$ with $\left|\xi_{h}(z)\right| \leq 1$. Moreover, we set $\kappa:=h / 2$. The nearest neighbour projection $\pi_{T_{1,1 / 4}}$ was approximated via a reformulation as a saddle-point problem; cf. 17, 8 for details. In Figure 5 we display the deformed triangulations

$$
\mathcal{T}_{h}^{i}:=\left\{u_{h}^{i}(K): K \in \mathcal{T}_{h}\right\}
$$

after various numbers of iterations. We see that the discrete flow defined by Algorithm A selects an approximation of the identity map (up to a rotation) on $T_{1,1 / 4}$ yielding a smooth regularization of the rough initial data. We note that the identity map id: $M \rightarrow M$ is a harmonic map from $M$ into $M$ since $\Delta_{M}$ id $(x)=H(x) \mu(x)$ for all $x \in M$ with $H(x)$ and $\Delta_{M}$ denoting the (scalar) mean curvature and the Laplace-Beltrami operator on $M$, respectively. For other target manifolds such as the unit sphere it is advantageous to factor out Möbius transformations in order to improve the stability of numerical methods; cf. [14] for related details.

\section{Appendix A. WeAK COMPaCtNESS RESUlts}

We briefly outline the weak compactness result due to 23 which serves as a guideline for the analysis of finite element approximations. Suppose that $\left(u_{\ell}\right)_{\ell \in \mathbb{N}} \subset$ $W^{1,2}\left(M ; \mathbb{R}^{n}\right)$ is a bounded sequence of almost harmonic maps into $N$. Thus, for each $\ell \in \mathbb{N}$ we have $u_{\ell}(x) \in N$ for almost every $x \in M,\left\|\nabla u_{\ell}\right\| \leq C$, and

$$
\left(\nabla u_{\ell} ; \nabla v\right)=\left\langle R_{\ell} ; v\right\rangle
$$

for all $v \in W_{0}^{1,2}\left(M ; \mathbb{R}^{n}\right)$ such that $v(x) \in T_{u_{\ell}(x)} N$ for almost every $x \in M$ and a sequence $\left(R_{\ell}\right)_{\ell \in \mathbb{N}} \subset W^{1,2}\left(M ; \mathbb{R}^{n}\right)^{*}$ satisfying $R_{\ell} \rightarrow 0$ as $\ell \rightarrow \infty$. 
If $N=S^{n-1}$ is the unit sphere in $\mathbb{R}^{n}$, then every $v \in W_{0}^{1,2}\left(M ; \mathbb{R}^{n}\right) \cap L^{\infty}\left(M ; \mathbb{R}^{n}\right)$ satisfying $v(x) \in T_{u_{\ell}(x)} S^{n-1}$ for almost every $x \in M$ can be written as $v=u_{\ell} \wedge w$ for a function $w \in W_{0}^{1,2}\left(M ; \Lambda^{2}\left(\mathbb{R}^{n}\right)\right) \cap L^{\infty}\left(M ; \Lambda^{2}\left(\mathbb{R}^{n}\right)\right)$, with $\Lambda^{2}\left(\mathbb{R}^{n}\right)$ denoting the set of alternating bilinear forms on $\mathbb{R}^{n}$, and the identity

$$
\left(\nabla u_{\ell} ; \nabla v\right)=\left(\nabla u_{\ell} ; \nabla\left[u_{\ell} \wedge w\right]\right)=\left(\nabla u_{\ell} ; u_{\ell} \wedge \nabla w\right)
$$

leads to an equivalent characterization of harmonic maps into $S^{n-1}$, which, by compactness of the embedding of $W^{1,2}$ into $L^{2}$, implies a weak compactness result.

The case of a general target manifold is significantly harder, and we outline the result of [23] for parallelizable target manifolds. By restricting to a cube $Q \subset M$ and reflecting $u$ across the sides of $Q$ one may assume that each $u_{\ell}$ is periodic; see 23 and Section 5 for details about this argument. For each $\ell \in \mathbb{N}$ let $\left(e_{\ell}^{i}\right)_{i=1,2, \ldots, k} \subset W^{1,2}\left(M ; \mathbb{R}^{n}\right)$ be an orthonormal frame for $u_{\ell}^{-1} T N$. Choosing $v=\eta e_{\ell}^{i}$ and expanding the rows of $\nabla u$ in the basis $\left(e_{\ell}^{i}\right)_{i=1,2, \ldots, k}$, one deduces that with $\omega_{\ell}^{i j}:=e_{\ell}^{j, \mathrm{~T}} \nabla e_{\ell}^{i}$ and $\vartheta_{\ell}^{j}:=e_{\ell}^{j, \mathrm{~T}} \nabla u_{\ell}$ the identity

$$
\sum_{j=1}^{k}\left(\omega_{\ell}^{i j} \cdot \vartheta_{\ell}^{j} ; \eta\right)+\left(\vartheta_{\ell}^{i} ; \nabla \eta\right)=\left\langle R_{\ell} ; \eta e_{\ell}^{i}\right\rangle
$$

is satisfied for all $\eta \in C_{c}^{\infty}(M)$ and each $\ell \in \mathbb{N}$. With a Coulomb gauge of the frame $\left(e_{\ell}^{i}\right)$ one has $\operatorname{div} \omega_{\ell}^{i j}=0$ so that $\omega_{\ell}^{i j}=\operatorname{Curl} b_{\ell}^{i j}+H_{\ell}^{i j}$ with periodic functions $b_{\ell}^{i j} \in$ $W^{1,2}(M)$ and harmonic fields $H_{\ell}^{i j} \in L^{2}\left(M ; \mathbb{R}^{2}\right)$ satisfying $\left\|\operatorname{Curl} b_{\ell}^{i j}\right\|^{2}+\left\|H_{\ell}^{i j}\right\|^{2}=$ $\left\|\omega_{\ell}^{i j}\right\|^{2}$. Then, (33) can be written as

$$
\sum_{j=1}^{k}\left\{\left(\operatorname{Curl} b_{\ell}^{i j} \cdot \vartheta_{\ell}^{j} ; \eta\right)+\left(H_{\ell}^{i j} \cdot \vartheta_{\ell}^{j} ; \eta\right)\right\}+\left(\vartheta_{\ell}^{i} ; \nabla \eta\right)=\left\langle R_{\ell}, \eta e_{\ell}^{i}\right\rangle .
$$

Let $u \in W^{1,2}\left(M ; \mathbb{R}^{n}\right)$ be a weak accumulation point of the sequence $\left(u_{\ell}\right)$ so that for a subsequence, which is not relabeled in the following, one has $u_{\ell} \rightarrow u$ in $W^{1,2}\left(M ; \mathbb{R}^{n}\right), u_{\ell} \rightarrow u$ in $L^{2}\left(M ; \mathbb{R}^{n}\right)$ and, since $N$ is continuous, $u(x) \in N$ for almost every $x \in M$. Since $\left(e_{\ell}^{i}\right)_{\ell \in \mathbb{N}}$ is bounded in $W^{1,2}\left(M ; \mathbb{R}^{n}\right)$, it follows that (after extraction of another subsequence) $e_{\ell}^{i} \rightarrow e^{i}$ in $W^{1,2}\left(M ; \mathbb{R}^{n}\right)$ for $i=1,2, \ldots, k$ and thus $\left(e^{i}\right)_{i=1,2, \ldots, k}$ is an orthonormal frame for $u^{-1} T N$. Moreover, one deduces that, as $\ell \rightarrow \infty$,

$$
\begin{array}{ll}
\omega_{\ell}^{i j} \rightarrow \omega^{i j}=e^{i, \mathrm{~T}} \nabla e^{j} \quad \text { in } L^{2}\left(M ; \mathbb{R}^{2}\right), & \vartheta_{\ell}^{j} \rightarrow \vartheta^{j}=e^{j, \mathrm{~T}} \nabla u \quad \text { in } L^{2}\left(M ; \mathbb{R}^{2}\right), \\
b_{\ell}^{i j} \rightarrow b^{i j} \quad \text { in } W^{1,2}(M), & H_{\ell}^{i j} \rightarrow H^{i j} \quad \text { in } L^{2}\left(M ; \mathbb{R}^{2}\right),
\end{array}
$$

since $\left(H_{\ell}^{i j}\right)_{\ell \in \mathbb{N}}$ belongs to a finite-dimensional subspace of $L^{2}\left(M ; \mathbb{R}^{n}\right)$. Therefore,

$$
\omega^{i j}=\operatorname{Curl} b^{i j}+H^{i j} .
$$

Passage to the limit in (34) as $\ell \rightarrow \infty$ is now straightforward for the right-hand side as well as for the second and third terms on the left-hand side of (34). To identify the limit of the first term, one notices that by definition of $\vartheta_{\ell}^{i j}$, it follows that

$$
\operatorname{Curl} b_{\ell}^{i j} \cdot \vartheta_{\ell}^{j}=\sum_{\alpha=1}^{n} e^{j, \alpha} \operatorname{Curl} b_{\ell}^{i j} \cdot \nabla u_{\ell}^{\alpha}
$$


and the right-hand side has a Jacobian structure. Thus, a result from concentration and compensation compactness, see [32], based on Wente's inequality in a periodic setting, implies that

$$
\operatorname{Curl} b_{\ell}^{i j} \cdot \vartheta_{\ell}^{j} \rightarrow \operatorname{Curl} b^{i j} \cdot \vartheta^{j}+\sum_{\iota \in \mathbb{N}} s_{\iota} \delta_{x_{\iota}}
$$

in the sense of distributions; the reader is referred to [23] for a detailed discussion. Here, $\left(x_{\iota}\right)_{\iota \in \mathbb{N}} \subset M$, and $\left(s_{\iota}\right)_{\iota \in \mathbb{N}} \subseteq \mathbb{R}$ satisfies $\sum_{\iota \in \mathbb{N}}\left|s_{\iota}\right|<\infty$. A combination of the limits identified above implies that

$$
\sum_{j=1}^{k}\left(\omega^{i j} \cdot \vartheta^{j} ; \eta\right)+\left(\vartheta^{j} ; \nabla \eta\right)=\sum_{\iota \in \mathbb{N}} s_{\iota} \eta\left(x_{\iota}\right)
$$

for all $\eta \in C_{c}^{\infty}(M)$. Since the left-hand side of (35) belongs to $L^{1}(M)+H^{-1}(M)$, which does not contain Dirac measures, one can show that the right-hand side of (35) has to vanish identically, i.e., $s_{\iota}=0$ for all $\iota \in \mathbb{N}$. Again the reader is referred to 23] for details. Proposition 2.1 implies that $u$ is a harmonic map into $N$ and concludes this outline of the compactness result into general targets due to 23 .

We remark that a new, more direct, compactness result for harmonic maps into $C^{2}$ targets $N$ has recently been established in [37] and avoids the use of a moving frame. Though weak compactness results for harmonic maps are only available for two-dimensional settings, no examples for failure of weak compactness of harmonic maps in higher dimensions are known.

\section{Appendix B. Auxiliary Results from measure theory}

We include two elementary results from measure theory. The first result states that the space of linear combinations of Dirac measures is a closed subset of $C(\bar{M})^{*}$ with respect to the strong topology, while the second one allows us to identify the supports of the accumulation points of certain sequences in $C(\bar{M})^{*}$.

Lemma B.1. Let $\left(F_{\ell}\right)_{\ell \in \mathbb{N}}$ be a bounded sequence in $C(\bar{M})^{*}$. If for each $\ell \in \mathbb{N}$ the support of $F_{\ell}$ is finite, i.e., $F_{\ell}=\sum_{j=1}^{L_{\ell}} a_{j}^{\ell} \delta_{x_{j}^{\ell}}$ for $L_{\ell} \in \mathbb{N}$ and $a_{j}^{\ell} \in \mathbb{R}, x_{j}^{\ell} \in \bar{M}$, $j=1,2, \ldots, L_{\ell}$, and if $F_{\ell} \rightarrow F$ strongly as $\ell \rightarrow \infty$ for some $F \in C(\bar{M})^{*}$, i.e.,

$$
\sup _{\eta \in C(\bar{M}):\|\eta\|_{L^{\infty}(M)} \leq 1}\left\langle F_{\ell}-F, \eta\right\rangle \rightarrow 0
$$

as $\ell \rightarrow \infty$, then there exist $\left(a_{j}\right)_{j \in \mathbb{N}} \subset \mathbb{R}$ and $\left(x_{j}\right)_{j \in \mathbb{N}} \subset \bar{M}$ such that $F=$ $\sum_{j=1}^{\infty} a_{j} \delta_{x_{j}}$. If $\sum_{j=1}^{L_{\ell}}\left|a_{j}^{\ell}\right|^{s} \leq C_{1}$ for some $s>0$ and all $\ell \in \mathbb{N}$, then $\sum_{j=1}^{\infty}\left|a_{j}\right|^{s} \leq$ $C_{1}$.

Proof. Riesz' representation theorem, see, e.g., [38, Theorem 6.19] for details, provides an isometric isomorphism between $C(\bar{M})^{*}$ and the set of regular Borel measures on $\bar{M}$ such that for every $G \in C(\bar{M})^{*}$ we have

$$
\sup _{\eta \in C(\bar{M}):\|\eta\|_{L^{\infty}(M)} \leq 1}\langle G, \eta\rangle=\sup _{\left\{E_{k}: k \in \mathbb{N}\right\} \in \mathcal{P}(\bar{M})} \sum_{k=1}^{\infty}\left|G\left(E_{k}\right)\right|,
$$


where we identified $G$ with the measure provided by the isomorphism and where $\mathcal{P}(\bar{M})$ denotes the set of all countable, measurable partitions of $\bar{M}$. The set

$$
\Gamma:=\bigcup_{\ell \in \mathbb{N}}\left\{x_{1}^{\ell}, x_{2}^{\ell}, \ldots, x_{L_{\ell}}^{\ell}\right\}
$$

is countable and we enumerate its elements as $\Gamma=\left\{x_{1}, x_{2}, x_{3}, \ldots\right\}$. We set $a_{j}:=$ $F\left(\left\{x_{j}\right\}\right)$ for $j \in \mathbb{N}$ and define $F^{\prime}:=\sum_{j=1}^{\infty} a_{j} \delta_{x_{j}} \in C(\bar{M})^{*}$. To finish the proof of the first statement it suffices to show that $F$ is supported on $\Gamma$ since this implies $F^{\prime}=F$. Each $F_{\ell}$ is supported on $\Gamma$, and thus for every measurable set $A \subset \bar{M} \backslash \Gamma$ we have by considering the partition $\{A, \bar{M} \backslash A\}$ in (36) that

$$
|F(A)|=\left|F(A)-F_{\ell}(A)\right| \leq \sup _{\eta \in C(\bar{M}):\|\eta\|_{L^{\infty}(M)} \leq 1}\left\langle F_{\ell}-F, \eta\right\rangle
$$

and the right-hand side can be made arbitrarily small, i.e., $F(A)=0$. To prove the second part of the lemma we first notice that for every $x \in \bar{M}$ we have

$$
\left|F_{\ell}(\{x\})-F(\{x\})\right| \rightarrow 0
$$

as $\ell \rightarrow \infty$. With Fatou's lemma we then deduce that

$$
\begin{aligned}
C_{1} & \geq \liminf _{\ell \rightarrow \infty} \sum_{j=1}^{L_{\ell}}\left|a_{j}^{\ell}\right|^{s}=\liminf _{\ell \rightarrow \infty} \sum_{j=1}^{\infty}\left|F_{\ell}\left(\left\{x_{j}\right\}\right)\right|^{s} \\
& \geq \sum_{j=1}^{\infty} \liminf _{\ell \rightarrow \infty}\left|F_{\ell}\left(\left\{x_{j}\right\}\right)\right|^{s}=\sum_{j=1}^{\infty}\left|F\left(\left\{x_{j}\right\}\right)\right|^{s}=\sum_{j=1}^{\infty}\left|a_{j}\right|^{s},
\end{aligned}
$$

which finishes the proof of the lemma.

Lemma B.2. Let $\left(F_{h}\right)_{h>0}$ be a bounded sequence in $C(\bar{M})^{*}$. Suppose that there exist $C>0$ and $L \in \mathbb{N}$ such that for each $h>0$ and all $\eta \in C^{1}(\bar{M})$ we have

$$
\left|F_{h}(\eta)\right| \leq C h\|\nabla \eta\|+\sum_{j=1}^{L} \varrho_{j}^{h}\left|\eta\left(x_{j}^{h}\right)\right|
$$

for $\varrho_{j}^{h} \in \mathbb{R}$ and $x_{j}^{h} \in \bar{M}$ for $j=1,2, \ldots, L$. Then there exist $L^{\prime} \leq L$ and $\varrho_{j} \in \mathbb{R}$, $y_{j} \in \bar{M}, j=1,2, \ldots, L^{\prime}$ such that for a subsequence which is not relabeled we have

$$
F_{h} \rightarrow^{*} \sum_{j=1}^{L^{\prime}} \varrho_{j} \delta_{y_{j}}
$$

as $h \rightarrow 0$. If $s \in(0,1]$ and $\sum_{j=1}^{L}\left|\varrho_{j}^{h}\right|^{s} \leq C_{1}$ for all $h>0$, then $\sum_{j=1}^{L^{\prime}}\left|\varrho_{j}\right|^{s} \leq C_{1}$.

Proof. Since $F_{h}$ is a bounded sequence in $C(\bar{M})^{*}$ there exists a weak limit $F \in$ $C(\bar{M})^{*}$ of a subsequence, which we do not relabel in the following. Passing to another subsequence we may assume that the $L$-tuples $\left(x_{1}^{h}, \ldots, x_{L}^{h}\right)$ converge strongly to $\left(x_{1}, \ldots, x_{L}\right) \in \bar{M}^{L}$ as $h \rightarrow 0$. For $\eta \in C^{1}(\bar{M})$ with $\operatorname{supp} \eta \subset \bar{M} \backslash\left\{x_{1}, \ldots, x_{L}\right\}$ we have, owing to the assumptions on $F_{h}$, that

$$
\begin{aligned}
|F(\eta)| & \leq\left|F(\eta)-F_{h}(\eta)\right|+\left|F_{h}(\eta)\right| \\
& \leq\left|F(\eta)-F_{h}(\eta)\right|+C h\|\nabla \eta\|+\sum_{j=1}^{L} \varrho_{j}^{h}\left|\eta\left(x_{j}^{h}\right)\right| .
\end{aligned}
$$


The right-hand side vanishes as $h \rightarrow 0$ owing to $F_{h} \rightarrow^{*} F$, convergence of $\left(x_{1}^{h}, \ldots, x_{L}^{h}\right)$ to $\left(x_{1}, \ldots, x_{L}\right)$, and the fact that $\eta$ vanishes in an open neighbourhood of $\left\{x_{1}, \ldots, x_{L}\right\}$. Therefore, we deduce that $F$ is supported on $\left\{x_{1}, \ldots, x_{L}\right\}$, i.e.,

$$
F=\sum_{j=1}^{L^{\prime}} \varrho_{j} \delta_{y_{j}}
$$

for appropriate $L^{\prime} \leq L, \varrho_{j} \in \mathbb{R}, j=1,2, \ldots, L^{\prime}$, and $\left\{y_{1}, \ldots, y_{L^{\prime}}\right\} \subseteq\left\{x_{1}, \ldots, x_{L}\right\}$. We set $\varepsilon:=\min _{i, j=1, \ldots, L^{\prime}}\left|y_{i}-y_{j}\right| / 2$. For $i \in\left\{1, \ldots, L^{\prime}\right\}$ we choose $\eta_{i} \in C^{1}(\bar{M})$ such that $\left|\eta_{i}(x)\right| \leq 1$ for all $x \in \bar{M}, \eta_{i}\left(y_{i}\right)=1$ and $\eta_{i}\left(y_{j}\right)=0$ for $j \neq i$, supp $\eta_{i} \subseteq B_{\varepsilon}\left(y_{i}\right) \cap \bar{M}$, and $\left\|\nabla \eta_{i}\right\| \leq C \varepsilon^{-1}$. Then, for each $h>0$ we have

$$
\begin{aligned}
\left|\varrho_{i}\right| & =\left|F\left(\eta_{i}\right)\right| \leq\left|F\left(\eta_{i}\right)-F_{h}\left(\eta_{i}\right)\right|+\left|F_{h}\left(\eta_{i}\right)\right| \\
& \leq\left|F\left(\eta_{i}\right)-F_{h}\left(\eta_{i}\right)\right|+C h \varepsilon^{-1}+\sum_{j=1, \ldots, L,\left|y_{i}-x_{j}^{h}\right| \leq \varepsilon} \varrho_{j}^{h} \\
& \leq\left(\left|F\left(\eta_{i}\right)-F_{h}\left(\eta_{i}\right)\right|^{s}+\left(C h \varepsilon^{-1}\right)^{s}+\sum_{j=1, \ldots, L:\left|y_{i}-x_{j}^{h}\right| \leq \varepsilon}\left|\varrho_{j}^{h}\right|^{s}\right)^{1 / s},
\end{aligned}
$$

where we used $[z]_{\ell^{1}} \leq[z]_{\ell^{s}}$ for $\left(z_{j}\right)_{j \in \mathbb{N}} \subset \mathbb{R}$ and $[z]_{\ell^{s}}:=\left(\sum_{j \in \mathbb{N}}\left|z_{j}\right|^{s}\right)^{1 / s}$. For each $x_{j}^{h}$ there is at most one $i$ such that $x_{j}^{h} \in B_{\varepsilon}\left(y_{i}\right)$. Therefore, we deduce that

$$
\sum_{i=1}^{L^{\prime}}\left|\varrho_{i}\right|^{s} \leq \sum_{i=1}^{L^{\prime}}\left|F\left(\eta_{i}\right)-F_{h}\left(\eta_{i}\right)\right|^{s}+L^{\prime}\left(C h \varepsilon^{-1}\right)^{s}+\sum_{j=1}^{L}\left|\varrho_{j}^{h}\right|^{s} .
$$

Since the first two terms on the right-hand side vanish as $h \rightarrow 0$ and the third one is bounded by $C_{1}$, we verify the assertion of the lemma.

\section{ACKNOWLEDGMENTS}

The author wishes to thank Andreas Prohl, Christof Melcher, and Jan Kristensen for stimulating discussions and helpful remarks.

\section{REFERENCES}

[1] Alouges, F. A new algorithm for computing liquid crystal stable configurations: The harmonic mapping case. SIAM J. Numer. Anal. 34, 5 (1997), 1708-1726. MR.1472192 (98k:82190)

[2] Alouges, F. A new finite element scheme for Landau-Lifschitz equations. Discrete Contin. Dyn. Syst. Ser. S 1, 2 (2008), 187-196. MR2379897(2009a:65244)

[3] BALl, J. M. Orientable and non-orientable director fields for liquid crystals. In ICIAM 07 - 6th International Congress on Industrial and Applied Mathematics (Zurich, Switzerland, July 2007).

[4] Barrett, J. W., Bartels, S., Feng, X., and Prohl, A. A convergent and constraintpreserving finite element method for the $p$-harmonic flow into spheres. SIAM J. Numer. Anal. 45, 3 (2007), 905-927. MR2318794 (2008f:65170)

[5] Barrett, J. W. and Nürnberg, R. Finite element approximation of a Stefan problem with degenerate Joule heating. M2AN Math. Model. Numer. Anal. 38, 4 (2004), 633-652. MR2087727 (2005g:80014)

[6] Bartels, S. Robust a priori error analysis for the approximation of degree-one GinzburgLandau vortices. M2AN Math. Model. Numer. Anal. 39, 5 (2005), 863-882. MR2178565 (2006g:35114)

[7] Bartels, S. Stability and convergence of finite-element approximation schemes for harmonic maps. SIAM J. Numer. Anal. 43, 1 (2005), 220-238 (electronic). MR2177142 (2006j:65336) 
[8] BARTELs, S. Finite element approximation of harmonic maps between surfaces. Habilitation thesis, Humboldt-Universität zu Berlin, Germany, 2008.

[9] Bartels, S. and Prohl, A. Constraint preserving implicit finite element discretization of harmonic map flow into spheres, Math. Comp. 76, 260 (2007), 1847-1859. MR2336271 (2008j:65156)

[10] Bethuel, F. On the singular set of stationary harmonic maps. Manuscripta Math. 78, 4 (1993), 417-443. MR1208652 (94a:58047)

[11] Brezis, H., Coron, J.-M., And Lieb, E. H. Harmonic maps with defects. Comm. Math. Phys. 107, 4 (1986), 649-705. MR868739 (88e:58023)

[12] Christodoulou, D. And TAhvildar-Zadeh, A. S. On the regularity of spherically symmetric wave maps. Comm. Pure Appl. Math. 46, 7 (1993), 1041-1091. MR1223662 (94e:58030)

[13] Ciarlet, P. G. The finite element method for elliptic problems, vol. 40 of Classics in Applied Mathematics. Society for Industrial and Applied Mathematics (SIAM), Philadelphia, PA, 2002. MR 1930132

[14] Clarenz, U., AND Dziuk, G. Numerical methods for conformally parametrized surfaces. In CPDw04 - Interphase 2003: Numerical Methods for Free Boundary Problems (The Isaac Newton Institute for Mathematical Sciences, Cambridge, UK, April 2003).

[15] Clément, P. Approximation by finite element functions using local regularization. RAIRO Analyse Numérique 9, R-2 (1975), 77-84. MR0400739 (53:4569)

[16] de Gennes, P.-G., And Prost, J. The Physics of Liquid Crystals. Oxford Science Publications, Oxford, 1993. second ed.

[17] Demlow, A. and Dziuk, G. An adaptive finite element method for the Laplace-Beltrami operator on implicitly defined surfaces. SIAM J. Numer. Anal. 14 (2007), 421-442. MR2285862 (2008c:65320)

[18] DeSimone, A., Kohn, R. V., Müller, S., And Otto, F. Recent analytical developments in micromagnetics. in: Science of Hysteresis, Elsevier, G. Bertotti and I Magyergyoz, Eds. (2005).

[19] Dziuk, G. Finite elements for the Beltrami operator on arbitrary surfaces. In Partial differential equations and calculus of variations, vol. 1357 of Lecture Notes in Math. Springer, Berlin, 1988, pp. 142-155. MR976234 (90i:65194)

[20] Eells, J. And Lemaire, L. On the construction of harmonic and holomorphic maps between surfaces. Math. Ann. 252, 1 (1980), 27-52. MR590546 (81k:58030)

[21] Evans, L. C. Partial regularity for stationary harmonic maps into spheres. Arch. Rational Mech. Anal. 116, 2 (1991), 101-113. MR.1143435 (93m:58026)

[22] Frank, F. C. On the theory of liquid crystals. Discuss. Faraday Soc. 25 (1958), 19-28.

[23] Freire, A., Müller, S., And Struwe, M. Weak compactness of wave maps and harmonic maps. Ann. Inst. H. Poincaré Anal. Non Linéaire 15, 6 (1998), 725-754. MR.1650966 (2000a:58045)

[24] Hardt, R. M. Singularities of harmonic maps. Bull. Amer. Math. Soc. (N.S.) 34, 1 (1997), 15-34. MR.1397098 (98b:58046)

[25] HÉlein, F. Régularité des applications faiblement harmoniques entre une surface et une variété riemannienne. C. R. Acad. Sci. Paris Sér. I Math. 312, 8 (1991), 591-596. MR.1101039 (92e:58055)

[26] HÉlein, F. Harmonic maps, conservation laws and moving frames, second ed., vol. 150 of Cambridge Tracts in Mathematics. Cambridge University Press, Cambridge, 2002. MR.1913803 (2003g:58024)

[27] JäGER, W. AND KAUL, H. Rotationally symmetric harmonic maps from a ball into a sphere and the regularity problem for weak solutions of elliptic systems. J. Reine Angew. Math. 343 (1983), 146-161. MR705882 (85f:58031)

[28] Jost, J. On the existence of harmonic maps from a surface into the real projective plane. Compositio Math. 59, 1 (1986), 15-19. MR850117 (87k:58069)

[29] Landau, L. D. And Lifschitz, E. M. On the theory of the dispersion of magnetic permeability in ferromagnetic bodies. Phys. Z. Sovietunion 8 (1935), 153-169.

[30] Lin, F.-H. A remark on the map $x /|x|$. C. R. Acad. Sci. Paris Sér. I Math. 305, 12 (1987), 529-531. MR916327 (89b:58056)

[31] Lin, S. Y. AND LuSkin, M. Relaxation methods for liquid crystal problems. SIAM J. Numer. Anal. 26, 6 (1989), 1310-1324. MR1025090(90m:65106) 
[32] Lions, P.-L. The concentration-compactness principle in the calculus of variations. The limit case. I. Rev. Mat. Iberoamericana 1, 1 (1985), 145-201. MR834360 (87c:49007)

[33] Morrey, JR., C. B. Multiple integrals in the calculus of variations. Die Grundlehren der mathematischen Wissenschaften, Band 130. Springer-Verlag New York, Inc., New York, 1966. MR0202511 (34:2380)

[34] Müller, S., Struwe, M., And Šverák, V. Harmonic maps on planar lattices. Ann. Scuola Norm. Sup. Pisa Cl. Sci. (4) 25, 3-4 (1997), 713-730 (1998). MR1655538 (2000c:58030)

[35] Oseen, C. W. The theory of liquid crystals. Trans. Faraday Soc. 29 (1933), 883-899.

[36] Rivière, T. Everywhere discontinuous harmonic maps into spheres. Acta Math. 175, 2 (1995), 197-226. MR1368247 (96k:58059)

[37] Rivière, T. Conservation laws for conformally invariant variational problems. Invent. Math. 168, 1 (2007), 1-22. MR2285745 (2008d:58010)

[38] Rudin, W. Real and complex analysis, third ed., McGraw-Hill Book Co., New York, 1987. MR.924157 (88k:00002)

[39] Schoen, R., And Uhlenbeck, K. A regularity theory for harmonic maps. J. Differential Geom. 17, 2 (1982), 307-335. MR664498 (84b:58037a)

[40] Struwe, M. Variational methods, third ed., vol. 34 of Results in Mathematics and Related Areas. 3rd Series. A Series of Modern Surveys in Mathematics. Springer-Verlag, Berlin, 2000. MR.1736116 (2000i:49001)

[41] Vese, L. A. AND Osher, S. J. Numerical methods for $p$-harmonic flows and applications to image processing. SIAM J. Numer. Anal. 40, 6 (2002), 2085-2104 (electronic) (2003). MR.1974176 (2004k:65102)

[42] VirgA, E. G. Variational theories for liquid crystals, vol. 8 of Applied Mathematics and Mathematical Computation. Chapman \& Hall, London, 1994. MR1369095 (97m:73001)

Institute for Numerical Simulation, Rheinische Friedrich-Wilhelms-Universität Bonn, Wegelerstrasse 6, 53115 Bonn, Germany

E-mail address: bartels@ins.uni-bonn.de 